NIST Special Publication 260-199

\title{
Certification of Standard Reference Material $^{\circledR} 2925$ \\ Recombinant Human Serum Albumin Solution (Primary Reference Calibrator for Urine Albumin) (Frozen)
}

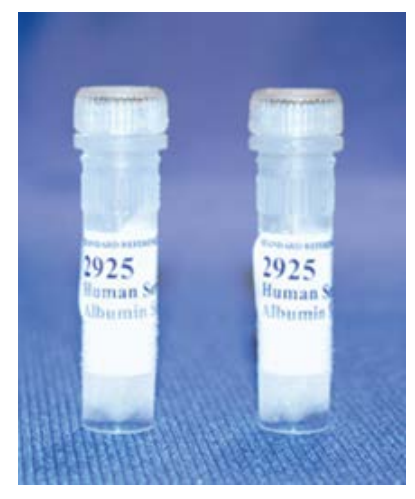

\author{
Ashley Beasley Green \\ David M. Bunk \\ Wendell Alejo \\ Nien Fan Zhang
}

This publication is available free of charge from: https://doi.org/10.6028/NIST.SP.260-199

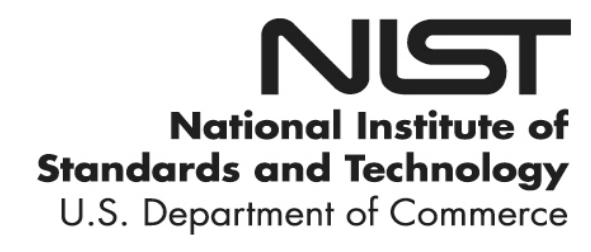


NIST Special Publication 260-199

\title{
Certification of Standard Reference Material $^{\circledR} 2925$ Recombinant Human Serum Albumin Solution (Primary Reference Calibrator for Urine Albumin) (Frozen)
}

\author{
Ashley Beasley Green \\ David M. Bunk \\ Wendell Alejo \\ Biomolecular Measurement Division \\ Material Measurement Laboratory \\ Nien Fan Zhang \\ Statistical Engineering Division \\ Information Technology Laboratory
}

This publication is available free of charge from:

https://doi.org/10.6028/NIST.SP.260-199

February 2020

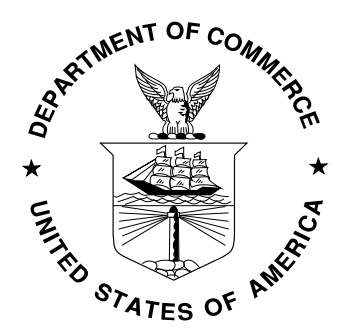

U.S. Department of Commerce Wilbur L. Ross, Jr., Secretary

National Institute of Standards and Technology Walter Copan, NIST Director and Undersecretary of Commerce for Standards and Technology 
Certain commercial entities, equipment, or materials may be identified in this document in order to describe an experimental procedure or concept adequately. Such identification is not intended to imply recommendation or endorsement by the National Institute of Standards and Technology, nor is it intended to imply that the entities, materials, or equipment are necessarily the best available for the purpose.

The opinions, recommendations, findings, and conclusions in this publication do not necessarily reflect the views or policies of NIST or the United States Government.

National Institute of Standards and Technology Special Publication 260-199

Natl. Inst. Stand. Technol. Spec. Publ. 260-199, 71 pages (February 2020)

CODEN: NSPUE2

This publication is available free of charge from: https://doi.org/10.6028/NIST.SP.260-199 


\begin{abstract}
SRM 2925 Recombinant Human Serum Albumin (Primary Reference Calibrator for Urine Albumin) (Frozen) is intended for use in calibration of liquid chromatography-tandem mass spectrometric procedures for the determination of human serum albumin (HSA). The concentration of recombinant HSA in this SRM was determined using amino acid analysis. A unit of SRM 2925 consists of two vials, each containing approximately $0.5 \mathrm{~mL}$ of solution. End users will need to evaluate the suitability of SRM 2925 for additional applications that require well-characterized protein for calibration or evaluation, such as colorimetric assays for total protein, gel diffusion, amino acid analysis, electrophoresis, or proteomics-based experimental workflows.
\end{abstract}

This publication documents the analytical methods and statistical evaluations involved in realizing this product.

\title{
Key words
}

Human serum albumin (HSA); Standard Reference Material (SRM); Reference Measurement Procedure.

\section{Technical Information Contact for this SRM}

Please address technical questions about this SRM to srms@nist.gov where they will be assigned to the appropriate Technical Project Leader responsible for support of this material. For sales and customer service inquiries, please contact srminfo@nist.gov. 


\section{TABLE OF CONTENTS}

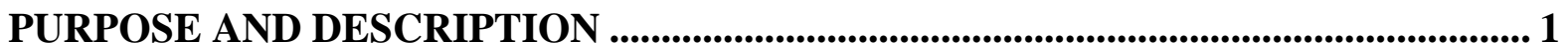

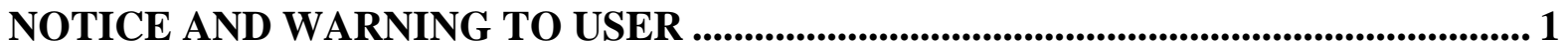

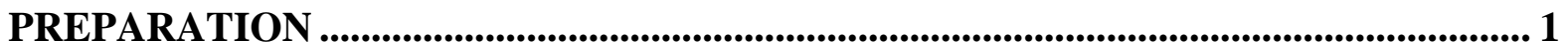

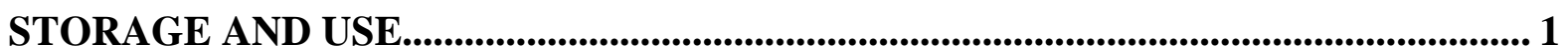

HOMOGENEITY AND STABILITY ANALYSIS .................................................... 1

CHARACTERIZATION OF THE MATERIAL _................................................. 2

Table 1: Certified Concentration Value for HSA in SRM 2925 by Amino Acid Analysis .. 2

Table 2: Non-Certified Values for Properties of SRM 2925 .................................................. 3

Table 3: Relative Molecular Mass Calculation of Mature, Native HSA ………………....... 4

CERTIFICATE OF ANALYSIS............................................................................. 5

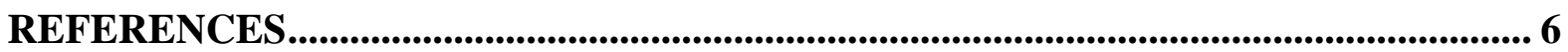

APPENDIX A: DATA SUBMITTED FOR STATISTICAL ANALYSIS........................... 7

Table A1: Amino acid analysis molar concentration values of recombinant HSA in SRM 2925 for three amino acids (Ala, Phe, Arg) ......................................................................... 7

APPENDIX B: REPORT OF STATISTICAL ANALYSIS ............................................. 8

Table B1. ANOVA table for measurements for Day and Sample Factors ............................ 8

Table B2. Protein Concentration averages for Day 1, 2, and 3........................................... 8

Figure B1. Box-and-whisker plot of measurements for Day Factor..................................... 8

Table B3. ANOVA table for measurements for Amino Acid Factor...................................... 9

Figure B2. Box-and-whisker plot of measurements for Amino Acid Factor......................... 9

Table B4. Type III Sums of Squares.............................................................................. 9

Table B5. Variance Components. ................................................................................. 9

APPENDIX C-G: EXTRACTS FROM SRM 2925 REPORTS OF ANALYSIS............. 12

APPENDIX C: Homogeneity and Stability Assessment of SRM 2925............................... 13

APPENDIX D: Certification of Total Protein content in SRM 2925 ................................... 23

APPENDIX E: Density Measurements of SRM 2925 ..................................................... 37

APPENDIX F: Assessment of Disulfide Bond Profile of SRM 2925 .................................. 39

APPENDIX G: Qualitative Characterization of SRM 2925 via Tandem Mass Spectrometry and 1-Dimensional Gel Electrophoresis. 


\section{PURPOSE AND DESCRIPTION}

SRM 2925 Recombinant Human Serum Albumin (Primary Reference Calibrator for Urine Albumin) (Frozen) is intended for use in calibration of liquid chromatography-tandem mass spectrometric procedures for the determination of human serum albumin (HSA). The concentration of recombinant HSA in this SRM was determined using amino acid analysis. A unit of SRM 2925 consists of two vials, each containing approximately $0.5 \mathrm{~mL}$ of solution.

End users will need to evaluate the suitability of SRM 2925 for additional applications that require well-characterized protein for calibration or evaluation, such as colorimetric assays for total protein, gel diffusion, amino acid analysis, electrophoresis, or proteomics-based experimental workflows.

\section{NOTICE AND WARNING TO USER}

SRM 2925 was expressed in Pichia pastoris yeast cells and has the potential to contain toxins that may pose a health risk. Normal caution and care should be exercised during the material's handling and use.

\section{PREPARATION}

SRM 2925 is a frozen aqueous solution of recombinant HSA, expressed in $P$. pastoris. The stock material was procured from Albumin Bioscience, a business unit of Albumin Therapeutics, LLC, (Huntsville, AL, USA) in a stock buffer composed of $5 \%$ solution of phosphate buffer saline containing $4 \mathrm{mmol} / \mathrm{L}$ sodium caprylate and $4 \mathrm{mmol} / \mathrm{L}$ acetyltryptophan. SRM 2925 was prepared at NIST by desalting the stock material via gel filtration chromatography $(3,000 \mathrm{~g} / \mathrm{mol}$ cutoff) and exchanging the stock buffer to $50 \mathrm{mmol} / \mathrm{L}$ ammonium bicarbonate in water.

\section{STORAGE AND USE}

SRM 2925 is shipped frozen (on dry ice) and, upon receipt, should be stored frozen until ready for use. A freezer temperature of $-20{ }^{\circ} \mathrm{C}$ is acceptable for storage for up to one week. If a longer storage time is anticipated, the material should be stored at or below $-80^{\circ} \mathrm{C}$. The SRM should not be exposed to sunlight or ultraviolet radiation. Storage of thawed material at room $\left(20^{\circ} \mathrm{C}\right.$ to $25^{\circ} \mathrm{C}$ ) or refrigerator $\left(5^{\circ} \mathrm{C}\right.$ to $8^{\circ} \mathrm{C}$ ) temperatures may result in degradation or modification of the constituent protein.

Frozen vials of the SRM should be removed from the freezer and allowed to stand on ice or at room temperature $\left(20^{\circ} \mathrm{C}\right.$ to $25^{\circ} \mathrm{C}$ ) until thawed. After the material is thawed, it should be used immediately. The material should be mixed briefly with a vortex mixer before aliquots are withdrawn.

\section{HOMOGENEITY AND STABILITY ANALYSIS}

Homogeneity was assessed prior to the certification analyses. A stratified sampling plan was devised to test for homogeneity across the lot of vials. There was no apparent trend in the data when plotted against the sequence in which the vials were filled. For homogeneity assessment results, see Appendix C. 
Stability was assessed prior to the certification analyses. A random sampling scheme was used to examine the degree of total protein degradation associated with the potential temperature conditions encountered during shipment from NIST to the end-user. There was no apparent trend in the data, which suggests that routine shipping conditions will not affect the composition of the material over a one-month period. For stability assessment results, see Appendix C.

\section{CHARACTERIZATION OF THE MATERIAL}

All analyses for the certified and non-certified values were performed at NIST. The amino acid analysis method involved isotope dilution-liquid chromatography tandem mass spectrometry (ID-LC-MS/MS) based on multiple reaction monitoring (MRM). For certification, randomly selected vials of SRM 2925 were combined with isotope-labeled analogs of alanine (Ala), phenylalanine (Phe), and arginine (Arg) and were hydrolyzed with vapor-phase hydrochloric acid $(\mathrm{HCl})$ for approximately $24 \mathrm{~h}$ at $120{ }^{\circ} \mathrm{C}$ in sealed vessels. After hydrolysis, the samples were dried down and reconstituted with $0.1 \mathrm{~mL} / \mathrm{L}$ hydrochloric acid $(\mathrm{HCl})$ in water. The amino acids were separated using gradient-elution mixed-mode chromatography on a reversed-phase analytical column with embedded acidic ion-pairing groups. Measurements were performed on a triple quadrupole mass spectrometer, monitoring a specific transition for each amino acid. The measurements were calibrated using amino acid certified reference materials from the National Metrology Institute of Japan (NMIJ; Tsukuba, Japan). For a detailed description of the amino acid analysis method, see Appendix D.

The certified concentration value for recombinant HSA in SRM 2925 is the total molar concentration of recombinant HSA calculated using the amount-of-substance determined for each of the three amino acids and the known amino acid sequence for HSA. Metrological traceability is to the SI units for molar concentration (expressed as nanomoles per gram). The certified concentration value of recombinant HSA in SRM 2925 is given in Table 1.

Table 1: Certified Concentration Value for HSA in SRM 2925 by Amino Acid Analysis

\begin{tabular}{cc} 
Material & $\begin{array}{c}\text { Certified Value } \\
( \pm \text { Expanded Standard Uncertainty })\end{array}$ \\
\hline \hline Recombinant HSA in SRM 2925 & $14.4 \mathrm{nmol} / \mathrm{g} \pm 0.3 \mathrm{nmol} / \mathrm{g} \quad(k=2)$ \\
\hline
\end{tabular}

The NIST certified value for recombinant HSA in SRM 2925 meets the formal, internationally accepted definitions for values delivered by Certified Reference Materials and the definition in $[1-4]$ :

"A NIST Certified Value represents data for which NIST has the highest confidence in its accuracy in that all known or suspected of bias have been fully investigated or accounted for by NIST."

The certified concentration for recombinant HSA was determined using a higher-order reference measurement procedure [4] calibrated with amino acid certified reference materials. The uncertainty provided value is an expanded uncertainty about the mean to cover the measurand, consistent with the ISO/JCGM Guide [3]. The expanded uncertainty is calculated as $U=k u_{\mathrm{c}}$, where $u_{\mathrm{c}}$ is the combined standard uncertainty, and $k$ is a coverage factor 
corresponding to approximately $95 \%$ confidence for this analyte [3]. For the certified value shown above, $k=2$.

The non-certified reference values and information value for recombinant HSA in SRM 2925 are listed in Table 2. Reference values are non-certified values that are best estimates of the true values; however, the values do not meet NIST criteria for certification and are provided with associated uncertainties that may reflect only measurement precision, may not include all sources of uncertainty, or may reflect a lack of sufficient statistical agreement among multiple analytical methods [2,3]. An information value is a value that may be of interest to the SRM user, but insufficient information is available to assess the uncertainty associated with the value [2]. Information values cannot be used to establish metrological traceability.

Table 2: Non-Certified Values for Properties of SRM 2925

\begin{tabular}{|c|c|}
\hline Property & Reference Value \\
\hline Density $\left(20.0^{\circ} \mathrm{C}\right)$ & $1.00016 \mathrm{~g} / \mathrm{mL} \quad \pm 0.00001 \mathrm{~g} / \mathrm{mL}$ \\
\hline Recombinant HSA Concentration in SRM 2925 & $0.958 \mathrm{~g} / \mathrm{L} \quad \pm 0.022 \mathrm{~g} / \mathrm{L}$ \\
\hline Property & Information Value \\
\hline Relative Molecular Mass (dimensionless) & 66394.56 \\
\hline
\end{tabular}

The reference value for density was determined volumetrically using the pulsed excitation method on the DMA 5000M Density Meter at $20.0{ }^{\circ} \mathrm{C}$. For a detailed description of the density measurements, see Appendix E.

The reference value for concentration of recombinant HSA in SRM 2925, expressed in terms of grams per liter, was calculated using the relative molecular mass (calculated from the amino acid sequence of mature, native HSA), the density value, and the certified recombinant HSA concentration value above in Table 1 . The non-certified information value for relative molecular mass is also included in Table 2. The relative molar mass was calculated from the atomic masses of the total number of carbon $\left({ }^{12} \mathrm{C}\right)$, nitrogen $\left({ }^{14} \mathrm{~N}\right)$, oxygen $\left({ }^{16} \mathrm{O}\right)$, sulfur $\left({ }^{32} \mathrm{~S}\right)$, and hydrogen $\left({ }^{1} \mathrm{H}\right)$ elements in each amino acid for the mature (residues 25-609) and native (including 17 disulfide linkages) amino acid sequence of HSA. As shown in Table 2, the theoretical molecular mass of HSA is $66,394.56 \mathrm{~g} / \mathrm{mol}$, which is based on the reported mature amino acid sequence and protein modifications (17 disulfide bonds). Calculation of the relative molecular mass of HSA is shown in Table 3. 
Table 3: Relative Molecular Mass Calculation of Mature, Native HSA

\begin{tabular}{ccccc}
\hline $\begin{array}{c}\text { Chemical } \\
\text { Element }\end{array}$ & $\begin{array}{c}\text { Total Number of } \\
\text { Chemical Elements } \\
\text { in HSA }\end{array}$ & $\begin{array}{c}\text { Mass of Chemical } \\
\text { Element }^{\mathbf{a}}(\mathbf{g} / \mathbf{m o l})\end{array}$ & $\begin{array}{c}\text { Total Mass of } \\
\text { Element in HSA } \\
\mathbf{( g / m o l})\end{array}$ & $\begin{array}{c}\text { Molecular Mass of } \\
\text { Mature, Native HSA } \\
\text { (g/mol) }\end{array}$ \\
\hline \hline Carbon $\left({ }^{\mathbf{1 2}} \mathbf{C}\right)$ & 2936 & 12.0000 & 35232.00 & $\mathbf{6 6 , 3 9 4 . 5 6}$ \\
\hline Nitrogen $\left({ }^{\mathbf{1 4}} \mathbf{N}\right)$ & 786 & 14.0031 & 11006.44 & \\
\hline Oxygen $\left({ }^{\mathbf{1 6}} \mathbf{O}\right)$ & 889 & 15.9949 & 14219.47 & \\
\hline Sulfur $\left({ }^{\mathbf{3 2}} \mathbf{S}\right)$ & 41 & 31.9721 & 1310.86 & \\
\hline Hydrogen $\left({ }^{\mathbf{1}} \mathbf{H}\right)$ & 4590 & 1.0078 & 4625.80 & \\
\hline
\end{tabular}

Qualitative characterization of recombinant HSA in SRM 2925 consisted of the following analyses: protein profile analysis via 1-dimensional gel electrophoresis; peptide profile analysis via tandem MS; and analysis of the stability of the native protein structure of the recombinant HSA via disulfide bond mapping. For a detailed description of the qualitative characterization of SRM 2925, see Appendix F and G. 


\section{CERTIFICATE OF ANALYSIS}

A NIST Certificate of Analysis (COA) is defined as:

"In accordance with ISO Guide 31: 2000, a NIST SRM certificate is a document containing the name, description, and intended purpose of the material, the logo of the U.S. Department of Commerce, the name of NIST as a certifying body, instructions for proper use and storage of the material, certified property value(s) with associated uncertainty(ies), method(s) used to obtain property values, the period of validity, if appropriate, and any other technical information deemed necessary for its proper use. A Certificate is issued for an SRM certified for one or more specific physical or engineering performance properties and may contain NIST reference, information, or both values in addition to certified values. A Certificate of Analysis is issued for an SRM certified for one or more specific chemical properties. Note: ISO Guide 31 is updated periodically; check with ISO for the latest version." [https://www.nist.gov/srm/srm-definitions] 


\section{REFERENCES}

1. JCGM 200:2012(E/F) International vocabulary of metrology - Basic and general concepts and associated terms (VIM). Joint Committee for Guides in Metrology. Sèvres, France (2012). http://www.bipm.org/en/publications/guides/vim.html

2. May W, Parris R, Beck C, Fassett J, Grenberg R, Guenther F, Kramer G, Wise S, Gills T, Colbert J, Gettings R, MacDonald B (2000). Definition of Terms and Modes Used at NIST for ValueAssignment of Reference Materials for Chemical Measurements. NIST Special Publication 260136. NIST, Gaithersburg, MD, USA. https://www.nist.gov/document-10363

3. JCGM 100:2008 Evaluation of measurement data -Guide to the expression of uncertainty in measurement. Joint Committee for Guides in Metrology. Sèvres, France (2008). http://www.bipm.org/en/publications/guides/\#gum

4. NCCLS; Development of Definitive Methods for the National Reference System for the Clinical Laboratory, Approved Guideline; NCCLS Publication NRSCL 1-A; National Committee for Clinical Laboratory Standards: Wayne, PA (1991).

5. Possolo A (2015) Simple Guide for Evaluating and Expressing the Uncertainty of NIST Measurement Results. NIST Technical Note 1900, NIST, Gaithersburg, MD, USA. http://doi.org/10.6028/NIST.TN.1900 See also: Possolo A (2015) Introducing a Simple Guide for the evaluation and expression of the uncertainty of NIST measurement results. Metrologia 53(1):S17-S24. http://doi.org/10.1088/0026-1394/53/1/S17

6. International Bureau of Weights and Measures (2006). The International System of Units (SI), $8^{\text {th }}$ Edition. BIPM, Sevres, France. https://www.bipm.org/en/publications/si-brochure/ 


\section{APPENDIX A: DATA SUBMITTED FOR STATISTICAL ANALYSIS}

Table A1 represents the data used in the statistical evaluation of the certified value listed in Table 1 of the Certificate of Analysis (COA) for SRM 2925.

Table A1: Amino acid analysis molar concentration values of recombinant HSA in SRM 2925 for three amino acids (Ala, Phe, Arg)

\begin{tabular}{|c|c|c|c|c|c|}
\hline & & & \multicolumn{3}{|c|}{ Recombinant HSA Concentration (nmol/g) } \\
\hline SRM 2925 Sample \# & Day & Technical Replicate & Alanine (Ala) & Phenylalanine (Phe) & Arginine (Arg) \\
\hline$\overline{1}$ & 1 & 1 & $\begin{array}{l}14.29 \\
\end{array}$ & 14.15 & 14.46 \\
\hline 1 & 1 & 2 & 14.45 & 14.17 & 14.29 \\
\hline 1 & 1 & 3 & 14.41 & 14.19 & 14.33 \\
\hline 1 & 1 & 4 & 14.30 & 14.28 & 14.38 \\
\hline 2 & 1 & 1 & 14.32 & 14.34 & 13.95 \\
\hline 2 & 1 & 2 & 14.36 & 14.26 & 13.84 \\
\hline 2 & 1 & 3 & 14.40 & 14.28 & 13.90 \\
\hline 2 & 1 & 4 & 14.38 & 14.30 & 13.94 \\
\hline 3 & 1 & 1 & 14.21 & 14.14 & 13.78 \\
\hline 3 & 1 & 2 & 14.15 & 14.11 & 13.77 \\
\hline 3 & 1 & 3 & 14.13 & 14.11 & 13.94 \\
\hline 3 & 1 & 4 & 14.15 & 14.14 & 13.64 \\
\hline 4 & 1 & 1 & 14.10 & 14.22 & 13.84 \\
\hline 4 & 1 & 2 & 14.28 & 14.18 & 13.89 \\
\hline 4 & 1 & 3 & 14.12 & 13.86 & 13.98 \\
\hline 4 & 1 & 4 & 14.22 & 14.17 & 13.87 \\
\hline 5 & 1 & 1 & 14.22 & 14.08 & 14.01 \\
\hline 5 & 1 & 2 & 14.29 & 14.09 & 13.97 \\
\hline 5 & 1 & 3 & 14.22 & 14.12 & 13.96 \\
\hline 5 & 1 & 4 & 14.26 & 14.25 & 13.93 \\
\hline 6 & 1 & 1 & 14.24 & 14.18 & 13.63 \\
\hline 6 & 1 & 2 & 14.34 & 14.00 & 13.66 \\
\hline 6 & 1 & 3 & 14.13 & 14.02 & 13.72 \\
\hline 6 & 1 & 4 & 14.37 & 14.14 & 13.57 \\
\hline 1 & 2 & 1 & 14.50 & 14.57 & 13.99 \\
\hline 1 & 2 & 2 & 14.36 & 14.49 & 14.14 \\
\hline 1 & 2 & 3 & 14.30 & 14.49 & 14.10 \\
\hline 1 & 2 & 4 & 14.47 & 14.59 & 13.94 \\
\hline 2 & 2 & 1 & 14.51 & 14.57 & 14.46 \\
\hline 2 & 2 & 2 & 14.37 & 14.62 & 14.36 \\
\hline 2 & 2 & 3 & 14.43 & 14.53 & 14.35 \\
\hline 2 & 2 & 4 & 14.54 & 14.54 & 14.40 \\
\hline 3 & 2 & 1 & 14.60 & 14.67 & 14.58 \\
\hline 3 & 2 & 2 & 14.61 & 14.66 & 14.51 \\
\hline 3 & 2 & 3 & 14.76 & 14.76 & 14.38 \\
\hline 3 & 2 & 4 & 14.45 & 14.60 & 14.47 \\
\hline 4 & 2 & 1 & 14.60 & 14.58 & 14.38 \\
\hline 4 & 2 & 2 & 14.71 & 14.63 & 14.41 \\
\hline 4 & 2 & 3 & 14.50 & 14.68 & 14.39 \\
\hline 4 & 2 & 4 & 14.74 & 14.62 & 14.41 \\
\hline 5 & 2 & 1 & 14.42 & 14.53 & 14.52 \\
\hline 5 & 2 & 2 & 14.29 & 14.48 & 14.10 \\
\hline 5 & 2 & 3 & 14.44 & 14.62 & 14.32 \\
\hline 5 & 2 & 4 & 14.43 & 14.55 & 14.33 \\
\hline 6 & 2 & 1 & 14.70 & 14.51 & 14.45 \\
\hline 6 & 2 & 2 & 14.53 & 14.47 & 14.50 \\
\hline 6 & 2 & 3 & 14.38 & 14.39 & 14.31 \\
\hline 6 & 2 & 4 & 14.46 & 14.55 & 14.35 \\
\hline 1 & 3 & 1 & 14.47 & 14.26 & 14.30 \\
\hline 1 & 3 & 2 & 14.43 & 14.55 & 14.20 \\
\hline 1 & 3 & 3 & 14.56 & 14.56 & 14.38 \\
\hline 1 & 3 & 4 & 14.49 & 14.48 & 14.41 \\
\hline 2 & 3 & 1 & 14.35 & 14.31 & 14.83 \\
\hline 2 & 3 & 2 & 14.63 & 14.28 & 14.51 \\
\hline 2 & 3 & 3 & 14.47 & 14.30 & 14.47 \\
\hline 2 & 3 & 4 & 14.27 & 14.29 & 14.50 \\
\hline 3 & 3 & 1 & 15.05 & 14.95 & 15.34 \\
\hline 3 & 3 & 2 & 15.11 & 14.94 & 15.22 \\
\hline 3 & 3 & 3 & 14.92 & 14.83 & 15.26 \\
\hline 3 & 3 & 4 & 14.93 & 14.88 & 15.39 \\
\hline 4 & 3 & 1 & 14.36 & 14.63 & 15.20 \\
\hline 4 & 3 & 2 & 14.58 & 14.39 & 14.82 \\
\hline 4 & 3 & 3 & 14.28 & 14.36 & $\begin{array}{l}14.96 \\
\end{array}$ \\
\hline 4 & 3 & 4 & 14.29 & 14.34 & 15.04 \\
\hline 5 & 3 & 1 & 14.79 & 14.80 & 15.39 \\
\hline 5 & 3 & 2 & 14.96 & 15.01 & 14.92 \\
\hline 5 & 3 & 3 & 14.79 & 14.81 & 15.10 \\
\hline 5 & 3 & 4 & 14.65 & 14.64 & 15.12 \\
\hline 6 & 3 & 1 & 14.43 & 14.30 & 15.69 \\
\hline 6 & 3 & 2 & 14.55 & 14.32 & 14.98 \\
\hline 6 & 3 & 3 & 14.32 & 14.30 & 15.10 \\
\hline 6 & 3 & 4 & 14.42 & 14.41 & 14.96 \\
\hline
\end{tabular}




\section{APPENDIX B: REPORT OF STATISTICAL ANALYSIS}

In this SRM for human serum albumin, the measurements were obtained through three amino acids (Alanine (1), Phenylalanine (2), Arginine (3)). The measurements were made on three days. For each day, six samples were used. For each sample, each of three acids was applied with four replicates. The data set is balanced.

\section{SRM 2925 CERTIFIED VALUE}

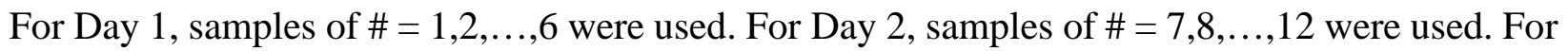
Day 3, samples of \# =13, 14,.., 18 were used. Altogether, there are 216 measurements. For the 216 measurements, the average is $14.4237 \mathrm{nmol} / \mathrm{g}$ and the standard error is 0.02347 .

For each amino acid, we checked the two factors of day and sample. From ANOVA - generalized linear models, the p-values for the factors of Day and Sample are listed in Table B1. Note that for each amino acid, the sample effect is nested within the day effect.

Table B1. ANOVA table for measurements for Day and Sample Factors

\begin{tabular}{ccccc}
\hline $\begin{array}{c}\text { Amino } \\
\text { acid }\end{array}$ & $\begin{array}{c}\text { Average } \\
\text { Concentration } \\
\text { (nmol/g) }\end{array}$ & $\begin{array}{c}\text { Standard deviation } \\
\text { (nmol/g) }\end{array}$ & $\begin{array}{c}\text { Day effect } \\
\text { (p-value) }\end{array}$ & $\begin{array}{c}\text { Sample effect } \\
\text { (p-value) }\end{array}$ \\
\hline \hline $\mathbf{1}$ & 14.4524 & 0.2227 & 0.01 & 0.0 \\
\hline $\mathbf{2}$ & 14.4227 & 0.2503 & 0.0 & 0.0 \\
$\mathbf{3}$ & 14.3959 & 0.4962 & 0.0 & 0.0 \\
\hline
\end{tabular}

The day effect is significant, and the sample effect is also significant for a given day. On the other hand, for each day, the acid effect is significant. The average values and the corresponding standard deviations and CV for each day are listed in Table B2. A box plot below Table 2 shows the mean difference of measurements for the three days (Figure B1). However, if we treat acid as the only factor for all the measurements, the effect of acid is not significant shown in Table 3 and a box plot (Figure B2) below Table B3.

Table B2. Protein Concentration averages for Day 1, 2, and 3.

\begin{tabular}{cccc}
\hline Day & $\begin{array}{c}\text { Average Concentration } \\
\text { (nmol/g) }\end{array}$ & $\begin{array}{c}\text { Standard deviation } \\
\text { (nmol/g) }\end{array}$ & $\begin{array}{c}\text { Coefficient } \\
\text { variation }\end{array}$ \\
\hline $\mathbf{1}$ & 14.1169 & 0.2121 & $1.50 \%$ \\
\hline $\mathbf{2}$ & 14.4716 & 0.1626 & $1.12 \%$ \\
\hline $\mathbf{3}$ & 14.6824 & 0.3508 & $2.39 \%$ \\
\hline
\end{tabular}

Figure B1. Box-and-whisker plot of measurements for Day Factor.

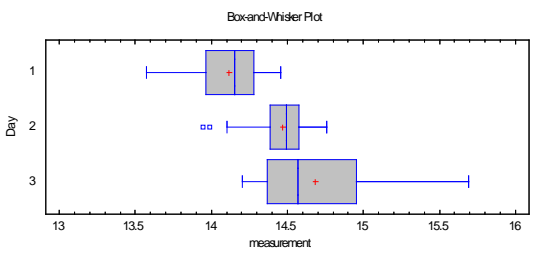


Table B3. ANOVA table for measurements for Amino Acid Factor.

\begin{tabular}{cccccc}
\hline Source & Sum of Squares & Df & Mean Square & F-Ratio & P-Value \\
\hline Between groups & 0.115077 & 2 & 0.0575383 & 0.48 & 0.6186 \\
\hline Within groups & 25.4563 & 213 & 0.119513 & & \\
\hline Total (Corr.) & 25.5714 & 215 & & & \\
\hline
\end{tabular}

Figure B2. Box-and-whisker plot of measurements for Amino Acid Factor.

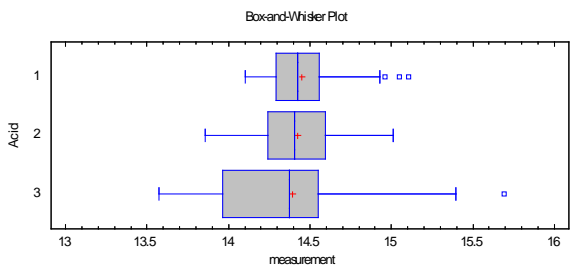

We consider a random effect model:

$$
X_{i j k l}=\mu+D_{i}+\alpha_{j}+S_{k(i j)}+\varepsilon_{i j k l},
$$

Equation B1

where $X_{i j k l}$ is the measurement for the $l$ th replicate on the $i$ th day for the $j$ th acid and $k$ th sample with $i=1,2,3, j=1,2,3, l=1,2,3,4$. In Eq. B1, $\mu$ is the mean. $D_{i}, \alpha_{j}$, and $S_{k(i j)}$ are the effects of the $i$ th day, $j$ th acid, and $k$ th sample, respectively. $\varepsilon_{i j k l}$ is the random error for the $i$ th day, $j$ th acid, $k$ th sample, and $l$ th replicate. The factor of sample is nested within the factors of day and acid. That is when $i=1$ and $j=1,2,3, k=1,2, \ldots, 6$. Similarly, when $i=2$ and $j=1,2,3, k=7,8, \ldots, 12$ and when $i=3$ and $j=1,2,3, k=13,14, . ., 18$. In the model in (1), we assume that the factors of day and sample are random while the factor of acid is fixed. The ANOVA in Table B4 gives

Table B4. Type III Sums of Squares.

\begin{tabular}{cccccc}
\hline Source & Sum of Squares & Df & Mean Square & F-Ratio & P-Value \\
\hline \hline Day & 11.7618 & 2 & 5.88092 & 34.02 & 0.0000 \\
\hline Amino Acid & 0.115077 & 2 & 0.0575383 & 0.33 & 0.7186 \\
\hline Sample (Day, Amino Acid) & 7.77843 & 45 & 0.172854 & 4.85 & 0.0000 \\
\hline Residual & 5.91604 & 166 & 0.0356388 & & \\
\hline Total (corrected) & 25.5714 & 215 & & & \\
\hline
\end{tabular}

It shows that the effects of day and sample are significant while the effect of acid is not significant. The variance components are given by

Table B5. Variance Components.

\begin{tabular}{cc}
\hline Source & Estimate \\
\hline \hline Day & 0.0792787 \\
\hline Sample (Day, Amino Acid) & 0.0343038 \\
\hline Residual & 0.0356388 \\
\hline
\end{tabular}


From Eq. B1,

$$
\bar{X}_{\ldots .}=\mu+\frac{\sum_{i=1}^{3} D_{i}}{3}+\frac{\sum_{j=1}^{3} \alpha_{j}}{3}+\frac{\sum_{i=1}^{3} \sum_{j=1}^{3} \sum_{k=1}^{6} S_{k(i, j)}}{54}+\frac{\sum_{i=1}^{3} \sum_{j=1}^{3} \sum_{k=1}^{6} \sum_{l=1}^{4} \varepsilon_{i j k l}}{216} .
$$

Equation B2

Based on the data, $\bar{X}_{\text {.... }}=14.4237$ is an estimate of $\mu$. From Eq B2,

$$
\operatorname{Var}\left[\bar{X}_{\ldots . . .}\right]=\frac{\sigma_{D}^{2}}{3}+\frac{\sigma_{S}^{2}}{54}+\frac{1}{216} \sigma_{\varepsilon}^{2} .
$$

Equation B3

From Table B5, the estimates of three variance components are given by $\hat{\sigma}_{D}^{2}=0.0792787, \hat{\sigma}_{S}^{2}=$ 0.0343088 , and $\hat{\sigma}_{\varepsilon}^{2}=0.0356388$. From Eq. B3, the standard uncertainty of $\bar{X}_{\ldots . . .}$ is $\hat{\sigma}_{\bar{X}} \ldots=$ $0.1650048 \mathrm{nmol} / \mathrm{g} . \bar{X}_{\text {.... }}=14.4237 \mathrm{nmol} / \mathrm{g}$. With a coverage factor of 2 , the expanded uncertainty of $\bar{X}_{\ldots . .}=2 * 0.1650048=0.3300096 \mathrm{nmol} / \mathrm{g}$.

Thus, the certified value of this SRM is $\bar{X}_{\ldots . . .}=14.4237 \mathrm{nmol} / \mathrm{g}$ with a standard uncertainty of

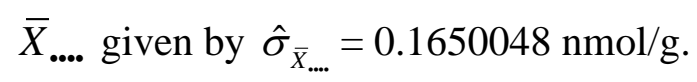

\section{SRM 2925 REFERENCE VALUE}

A formula for convert from nmol/g to g/L:

Reference value $(\mathrm{g} / \mathrm{L})=\left(\right.$ certified value $\left.(\mathrm{nmol} / \mathrm{g}) / 10^{6}\right)($ molecular mass $)($ Density $(\mathrm{g} / \mathrm{mL}))$

Given molecular mass $=66394.5608$ and density $=1.00016$,

Reference value $(\mathrm{g} / \mathrm{L})=\left(\right.$ certified value $\left.(\mathrm{nmol} / \mathrm{g}) / 10^{6}\right)($ molecular mass $)($ Density $(\mathrm{g} / \mathrm{mL}))$

$=0.0664 *$ certified value $* 1.00016$.

Equation B4

Given the certified value $\bar{X}_{\text {.... }}=14.4237 \mathrm{nmol} / \mathrm{g}$, the corresponding reference value $=0.9579 \mathrm{~g} / \mathrm{L}$.

From Eq. B4, the uncertainty of the reference value,

Reference value $=0.0664$. certified value $\cdot$ density .

Equation B5

From Eq. B5, using GUM’s approach,

$$
u_{r e f}=0.0664 \cdot \sqrt{d^{2} \cdot u_{c e r t}^{2}+\bar{X}_{\ldots .}^{2} \cdot u_{d}^{2}}
$$


where $d$ is the density and $u_{d}$ is its standard uncertainty. $u_{c e r t}$ is the standard uncertainty of the certified value from the above. Given $d=1.00016 \mathrm{~g} / \mathrm{mL}, u_{d}=0.00001 \mathrm{~g} / \mathrm{mL}$, and $\bar{X}_{\ldots . . .}=14.4237$ $\mathrm{nmol} / \mathrm{g}$,

$$
u_{\text {ref }}=0.0664 \cdot \sqrt{1.00016^{2} \cdot u_{\text {cert }}^{2}+14.4237^{2} \cdot 0.00001^{2}}
$$

Given $u_{\text {cert }}=\hat{\sigma}_{\bar{X}_{\text {.... }}}=0.1650048 \mathrm{nmol} / \mathrm{g}$, the standard uncertainty of the reference value $u_{\text {ref }}=$ $0.0110 \mathrm{~g} / \mathrm{L}$. With a coverage factor of 2 , the expanded uncertainty $=0.0220 \mathrm{~g} / \mathrm{L}$. 


\section{APPENDIX C-G: EXTRACTS FROM SRM 2925 REPORTS OF ANALYSIS}

A NIST Report of Analysis (ROA) is defined as:

"Document containing the certification of the material and including such information as the base material used, how the SRM was manufactured, the certification method(s) and description of procedures, outside collaborators, instructions for use, special instructions for packaging, handling, and storage, and plan for stability testing. The ROA is intended for internal NIST use only." [https://www.nist.gov/srm/srm-definitions]

The following pages have been extracted from the NIST ROAs that are directly related to the certification and characterization of NIST SRM 2925 Recombinant Human Serum Albumin (Primary Reference Calibrator for Urine Albumin) (Frozen). All information pertinent to the evaluation and use of the SRM has been retained. 


\section{APPENDIX C: Homogeneity and Stability Assessment of SRM 2925}

\section{INTRODUCTION}

This report utilizes liquid chromatographic (LC) separation with absorbance detection (LC-UV) to assess the intra- and inter-vial homogeneity and short-term stability of SRM 2925 Recombinant Human Serum Albumin (Primary Reference Calibrator for Urine Albumin) (Frozen). The goal of the homogeneity study is to assess the vial-to-vial uniformity of the material and identify variance in the composition of the standard reference materials that could potentially affect the intended use. In addition to lot homogeneity, assessment of SRM 2925 will indirectly evaluate the stability of the material during aliquoting. The shortterm stability study is a 1-month study to assess the degree of total protein degradation associated with the potential temperature conditions encountered during shipment from NIST to the end-user. The peak area is used to determine chromatographic purity, which in turn is used to assess the homogeneity and stability of SRM 2925.

\section{EXPERIMENTAL}

Homogeneity Study. For SRM 2925, a total of fifteen (15) vials were selected from the lot via a stratified random sampling scheme (Table C1). The LC-UV peak area for each sample was analyzed on three consecutive days and SRM 927d was used as the quality control material. See Table C2 for the certified recombinant HSA concentration for SRM 2925 and density.

Stability Study. The stability study was conducted over a 1-month period with the following time points: 1-month, 2-weeks, 1-week and 3-day. For SRM 2925, fifteen (15) samples at each time point (total of 52 samples) were randomly selected and three (3) samples were placed in the following temperatures until the end of the 1-month study: $-80^{\circ} \mathrm{C},-20^{\circ} \mathrm{C}, 5^{\circ} \mathrm{C}, 22^{\circ} \mathrm{C}$ (room temperature), and $37^{\circ} \mathrm{C}$ (Table C3). The LCUV peak area for each sample was measured on three consecutive days and SRM 927d was used as the quality control material.

Instrumental (LC-UV) Method. Samples were analyzed by using an Agilent 1100 series LC system composed of a mobile phase degasser, quaternary pump, autosampler, column oven, and photodiode array detector. An Agilent Zorbax 300SB-C8 column (2.1 x 150mm i.d., $3.5 \mu \mathrm{m}$ particles, Serial \#USAB001981) was used for the analyses and a gradient elution program (Table C4) was used with a flowrate of 0.2 $\mathrm{mL} / \mathrm{min}$. The column and autosampler were maintained at $22{ }^{\circ} \mathrm{C}$ (room temperature). Mobile phase A consisted of $0.1 \%(v / v)$ formic acid in water and mobile phase B was $0.1 \%(v / v)$ formic acid in acetonitrile. A volume of $1 \mu \mathrm{L}$ was injected onto the column and each sample was analyzed in replicate $(4 \mathrm{x})$. Elution was monitored at $214 \mathrm{~nm}$ using the photodiode array detector. The average purity at $214 \mathrm{~nm}$ was determined as an average of these four injections.

Quantitation. Quantitation was performed by direct comparison of the peak areas. The purity was calculated using Eq. 1, as the peak area for the compound of interest divided by the total peak area for all peaks observed in the chromatogram. Blank samples containing only $0.1 \%(v / v)$ formic acid in water were also injected to identify any carryover peaks between LC-UV runs.

Equations. The following equation was used in the homogeneity and stability assessment of SRM 2925. Equation 1: The material purity assessment for homogeneity and stability study:

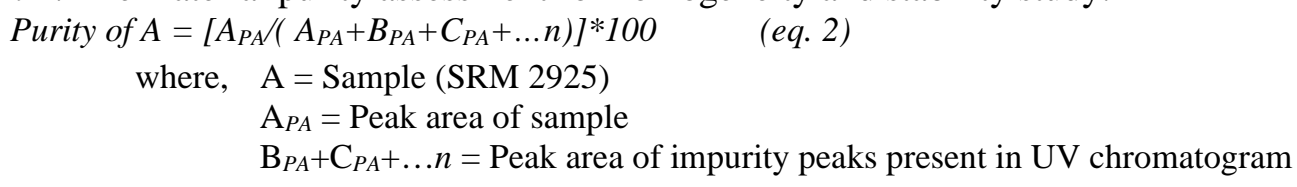




\section{RESULTS AND DISCUSSION}

Prior to analysis of the homogeneity and stability samples, the accuracy of the injection volume (Table C5) and precision of the LC-UV analysis (Table C6) were evaluated using SRM 927d Bovine Serum Albumin (7\% solution) as an external control. According to the vial mass measurements, the injection volume is reproducible across the six injections ( $5 \mu \mathrm{L})$ of SRM 927d (Table C5). To evaluate the precision of the peak area measurements, SRM 927d was injected over four days with six replicates each day (total of 24 runs). The repeatability observed in the intra- and inter-day peak area measurements for SRM 927d supports the high precision and accuracy of the LC-UV method. Figure C1 provides a visual representation of the repeatability of BSA elution in the SRM 927d LC-UV analyses.

The homogeneity study peak area measurements and purity calculations for SRM 2925 are shown in Table C7. Sample chromatograms for SRM 2925 with detection at $214 \mathrm{~nm}$ are shown in Figure C2. The high precision (low \%CV values) observed in the intra- and inter-vial peak area measurements support the repeatability of eluted HSA species in SRM 2925. No additional peaks were observed in the LC-UV chromatograms for the multiple vials of SRM 2925, which suggests that SRM 2925 is composed of only one species (recombinant HSA) and no factors impacted the material composition across the lot during aliquoting.

The stability study peak area measurements and purity calculations for SRM 2925 are shown in Table C8. Sample chromatograms for SRM 2925 with detection at $214 \mathrm{~nm}$ are shown in Figure C3. The stability peak area measurements (Table C8) for SRM 2925 have slightly elevated \% CV values, which implies that there is a difference in the measurement reproducibility between the two materials. No additional peaks were observed in the LC-UV chromatograms for SRM 2925, which supports that there is no degradative species produced at the elevated temperatures. The data for SRM 2925 suggests that the routine shipping conditions (on dry ice) will not affect the composition of the material. 
Table C1. SRM 2925 Homogeneity Study samples for LC-UV analysis.

\begin{tabular}{|c|c|c|}
\hline SRM 2925 Sample Name & Box Number & Box Location \\
\hline \hline $\mathbf{1 . 1}$ & Box 1.1 & Front 1 \\
\hline $\mathbf{1 . 2}$ & Box 1.2 & Front 2 \\
\hline $\mathbf{2 . 1}$ & Box 2.1 & Front 3 \\
\hline $\mathbf{3 . 1}$ & Box 3.1 & Front 4 \\
\hline $\mathbf{4 . 1}$ & Box 4.1 & Front 5 \\
\hline $\mathbf{5 . 1}$ & Box 5.1 & Middle 1 \\
\hline $\mathbf{5 . 2}$ & Box 5.2 & Middle 2 \\
\hline $\mathbf{7 . 1}$ & Box 7.1 & Middle 3 \\
\hline $\mathbf{7 . 2}$ & Box 7.2 & Middle 4 \\
\hline $\mathbf{8 . 1}$ & Box 8.1 & Middle 5 \\
\hline $\mathbf{1 0 . 1}$ & Box 10.1 & End 1 \\
\hline $\mathbf{1 1 . 1}$ & Box 11.1 & End 2 \\
\hline $\mathbf{1 2 . 1}$ & Box 12.1 & End 3 \\
\hline $\mathbf{1 2 . 2}$ & Box 12.2 & End 4 \\
\hline $\mathbf{1 2 . 3}$ & Box 12.3 & End 5 \\
\hline & & \\
\hline
\end{tabular}

Table C2. SRM 2925 total protein and density measurements.

\begin{tabular}{|c|c|c|}
\hline & Total Protein (g/L) & Density $(\mathbf{g} / \mathbf{m L})$ \\
\hline \hline SRM 2925 & $0.958 \pm 0.022$ & $1.00016 \pm 0.00001$ \\
\hline
\end{tabular}


Table C3. SRM 2925 Stability Study samples for LC-UV analysis.

\begin{tabular}{|c|c|c|c|}
\hline SRM 2925 Sample Name & Time Point & Temperature $\left({ }^{\circ} \mathrm{C}\right)$ & Sample Replicate \\
\hline 1-month -80-1 & 1-month & -80 & 1 \\
\hline 1-month -80-2 & 1-month & -80 & 2 \\
\hline 1-month -80-3 & 1-month & -80 & 3 \\
\hline 1-month -20-1 & 1-month & -20 & 1 \\
\hline 1-month -20-2 & 1-month & -20 & 2 \\
\hline 1-month -20-3 & 1-month & -20 & 3 \\
\hline 1-month 5-1 & 1-month & 5 & 1 \\
\hline 1-month 5-2 & 1-month & 5 & 2 \\
\hline 1-month 5-3 & 1-month & 5 & 3 \\
\hline 1-month RT-1 & 1-month & $22(\mathrm{RT})$ & 1 \\
\hline 1-month RT-2 & 1-month & $22(\mathrm{RT})$ & 2 \\
\hline 1-month RT-3 & 1-month & $22(\mathrm{RT})$ & 3 \\
\hline 1-month 37-1 & 1-month & 37 & 1 \\
\hline 1-month 37-2 & 1-month & 37 & 2 \\
\hline 1-month 37-3 & 1-month & 37 & 3 \\
\hline 2-week -20-1 & 2-week & -20 & 1 \\
\hline 2-week -20-2 & 2-week & -20 & 2 \\
\hline 2-week -20-3 & 2-week & -20 & 3 \\
\hline 2-week 5-1 & 2-week & 5 & 1 \\
\hline 2-week 5-2 & 2-week & 5 & 2 \\
\hline 2-week 5-3 & 2-week & 5 & 3 \\
\hline 2-week RT-1 & 2-week & 22 (RT) & 1 \\
\hline 2-week RT-2 & 2-week & 22 (RT) & 2 \\
\hline 2-week RT-3 & 2-week & 22 (RT) & 3 \\
\hline 2-week 37-1 & 2-week & 37 & 1 \\
\hline 2-week 37-2 & 2-week & 37 & 2 \\
\hline 2-week 37-3 & 2-week & 37 & 3 \\
\hline 1-week -20-1 & 1-week & -20 & 1 \\
\hline 1-week -20-2 & 1-week & -20 & 2 \\
\hline 1-week -20-3 & 1-week & -20 & 3 \\
\hline 1-week 5-1 & 1-week & 5 & 1 \\
\hline 1-week 5-2 & 1-week & 5 & 2 \\
\hline 1-week 5-3 & 1-week & 5 & 3 \\
\hline 1-week RT-1 & 1-week & 22 (RT) & 1 \\
\hline 1-week RT-2 & 1-week & $22(\mathrm{RT})$ & 2 \\
\hline 1-week RT-3 & 1-week & 22 (RT) & 3 \\
\hline 1-week 37-1 & 1-week & 37 & 1 \\
\hline 1-week 37-2 & 1-week & 37 & 2 \\
\hline 1-week 37-3 & 1-week & 37 & 3 \\
\hline 3-day -20-1 & 3-day & -20 & 1 \\
\hline 3-day -20-2 & 3-day & -20 & 2 \\
\hline 3-day -20-3 & 3-day & -20 & 3 \\
\hline 3-day 5-1 & 3-day & 5 & 1 \\
\hline 3-day 5-2 & 3-day & 5 & 2 \\
\hline 3-day 5-3 & 3-day & 5 & 3 \\
\hline 3-day RT-1 & 3-day & 22 (RT) & 1 \\
\hline 3-day RT-2 & 3-day & 22 (RT) & 2 \\
\hline 3-day RT-3 & 3-day & 22 (RT) & 3 \\
\hline 3-day 37-1 & 3-day & 37 & 1 \\
\hline 3-day 37-2 & 3-day & 37 & 2 \\
\hline 3-day 37-3 & 3-day & 37 & 3 \\
\hline
\end{tabular}

Table C4. LC gradient for Homogeneity and Stability study.

\begin{tabular}{|c|c|c|c|}
\hline $\begin{array}{c}\text { Time } \\
\text { (min.) }\end{array}$ & \% Solvent A & \% Solvent B & $\begin{array}{c}\text { Flowrate } \\
\text { (mL/min) }\end{array}$ \\
\hline \hline 0 & 97 & 3 & 0.2 \\
\hline 2 & 97 & 3 & 0.2 \\
\hline 4 & 50 & 50 & 0.2 \\
\hline 14 & 40 & 60 & 0.2 \\
\hline 15 & 3 & 97 & 0.2 \\
\hline 17 & 3 & 97 & 0.2 \\
\hline 18 & 97 & 3 & 0.2 \\
\hline 23 & 97 & 3 & 0.2 \\
\hline
\end{tabular}


Table C5. Injection repeatability of LC system using SRM 927d (Injection Precision Study)

\begin{tabular}{|c|c|c|c|}
\hline Injection \# & Vial Mass (g) & $\begin{array}{c}\text { Injection } \\
\text { Vol }(\mu L) \\
\end{array}$ & Injection Mass (g) \\
\hline 0 & 3.05708 & & \\
\hline 1 & 3.05203 & 5 & 0.00505 \\
\hline 2 & 3.04697 & 5 & 0.00506 \\
\hline 3 & 3.04192 & 5 & 0.00505 \\
\hline 4 & 3.03686 & 5 & 0.00506 \\
\hline 5 & 3.03179 & 5 & 0.00507 \\
\hline \multirow[t]{4}{*}{6} & 3.02673 & 5 & 0.00506 \\
\hline & \multicolumn{2}{|c|}{ Average Injection Mass } & 0.00506 \\
\hline & & Stdev & 0.00001 \\
\hline & & $\% C V$ & 0.1 \\
\hline
\end{tabular}

Table C6. Assessment of LC-UV (214 nm) instrumentation performance via SRM 927d (Measurement Precision Study)

\begin{tabular}{|c|c|c|c|c|c|c|c|c|c|}
\hline \multirow[b]{2}{*}{ Day } & \multirow[b]{2}{*}{$\begin{array}{l}\text { UV Trace } \\
\text { (nm) }\end{array}$} & \multirow[b]{2}{*}{ Run } & \multirow[b]{2}{*}{$\begin{array}{c}\text { Peak Area } \\
\text { (mAU) }\end{array}$} & \multicolumn{3}{|c|}{ Intra-Day Precision } & \multicolumn{3}{|c|}{ Inter-Day Precision } \\
\hline & & & & $\begin{array}{c}\text { Avg Peak Area } \\
\text { (mAU) }\end{array}$ & Stdev & $\% \mathrm{CV}$ & $\begin{array}{c}\text { Avg Peak Area } \\
\text { (mAU) }\end{array}$ & Stdev & $\% \mathrm{CV}$ \\
\hline \multirow[t]{6}{*}{$\overline{1}$} & 214 & 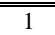 & $1.67 \mathrm{E}+04$ & $1.69 \mathrm{E}+04$ & 127.36 & 0.8 & $1.71 \mathrm{E}+04$ & 143.47 & 0.8 \\
\hline & 214 & 2 & $1.70 \mathrm{E}+04$ & & & & & & \\
\hline & 214 & 3 & $1.70 \mathrm{E}+04$ & & & & & & \\
\hline & 214 & 4 & $1.70 \mathrm{E}+04$ & & & & & & \\
\hline & 214 & 5 & $1.69 \mathrm{E}+04$ & & & & & & \\
\hline & 214 & 6 & $1.70 \mathrm{E}+04$ & & & & & & \\
\hline \multirow[t]{6}{*}{2} & 214 & 1 & $1.69 \mathrm{E}+04$ & $1.70 \mathrm{E}+04$ & 45.98 & 0.3 & & & \\
\hline & 214 & 2 & $1.70 \mathrm{E}+04$ & & & & & & \\
\hline & 214 & 3 & $1.70 \mathrm{E}+04$ & & & & & & \\
\hline & 214 & 4 & $1.70 \mathrm{E}+04$ & & & & & & \\
\hline & 214 & 5 & $1.70 \mathrm{E}+04$ & & & & & & \\
\hline & 214 & 6 & $1.69 \mathrm{E}+04$ & & & & & & \\
\hline \multirow[t]{6}{*}{3} & 214 & 1 & $1.70 \mathrm{E}+04$ & $1.71 \mathrm{E}+04$ & 88.95 & 0.5 & & & \\
\hline & 214 & 2 & $1.71 \mathrm{E}+04$ & & & & & & \\
\hline & 214 & 3 & $1.71 \mathrm{E}+04$ & & & & & & \\
\hline & 214 & 4 & $1.71 \mathrm{E}+04$ & & & & & & \\
\hline & 214 & 5 & $1.70 \mathrm{E}+04$ & & & & & & \\
\hline & 214 & 6 & $1.73 E+04$ & & & & & & \\
\hline \multirow[t]{6}{*}{4} & 214 & 1 & $1.72 \mathrm{E}+04$ & $1.72 \mathrm{E}+04$ & 112.37 & 0.7 & & & \\
\hline & 214 & 2 & $1.71 \mathrm{E}+04$ & & & & & & \\
\hline & 214 & 3 & $1.73 E+04$ & & & & & & \\
\hline & 214 & 4 & $1.73 E+04$ & & & & & & \\
\hline & 214 & 5 & $1.73 E+04$ & & & & & & \\
\hline & 214 & 6 & $1.71 \mathrm{E}+04$ & & & & & & \\
\hline
\end{tabular}


Table C7. SRM 2925 LC-UV (214 nm) results for Homogeneity Study.

\begin{tabular}{|c|c|c|c|c|c|c|c|c|}
\hline \multirow[b]{2}{*}{ SRM 2925 Sample Name } & \multicolumn{4}{|c|}{$\underline{\text { LC-UV Peak Area Measurements }}$} & \multicolumn{4}{|c|}{$\underline{\text { Intra-Vial Repeatability }}$} \\
\hline & $\begin{array}{c}\text { Technical Replicate } \\
1\end{array}$ & $\begin{array}{c}\text { Technical Replicate } \\
2\end{array}$ & $\begin{array}{c}\text { Technical Replicate } \\
3 \\
\end{array}$ & $\begin{array}{c}\text { Technical } \\
\text { Replicate } 4\end{array}$ & Avg. Peak Area (mAU) & Stdev & $\% \mathrm{CV}$ & $\begin{array}{c}\% \\
\text { Chromatographic } \\
\text { Purity }\end{array}$ \\
\hline 1.1 & $1.89 \mathrm{E}+04$ & $1.89 \mathrm{E}+04$ & $1.88 \mathrm{E}+04$ & $1.88 \mathrm{E}+04$ & $1.88 E+04$ & 34.75 & 0.2 & 100 \\
\hline 1.2 & $2.00 \mathrm{E}+04$ & $1.98 \mathrm{E}+04$ & $1.98 \mathrm{E}+04$ & $1.96 \mathrm{E}+04$ & $1.98 E+04$ & 179.96 & 0.9 & 100 \\
\hline 2.1 & $1.91 \mathrm{E}+04$ & $1.91 \mathrm{E}+04$ & $1.91 \mathrm{E}+04$ & $1.91 \mathrm{E}+04$ & $1.91 E+04$ & 15.63 & 0.1 & 100 \\
\hline 3.1 & $1.93 \mathrm{E}+04$ & $1.93 \mathrm{E}+04$ & $1.94 \mathrm{E}+04$ & $1.94 \mathrm{E}+04$ & $1.94 E+04$ & 42.47 & 0.2 & 100 \\
\hline 4.1 & $2.16 \mathrm{E}+04$ & $2.17 \mathrm{E}+04$ & $2.18 \mathrm{E}+04$ & $2.17 \mathrm{E}+04$ & $2.17 E+04$ & 87.30 & 0.4 & 100 \\
\hline 5.1 & $2.03 \mathrm{E}+04$ & $2.03 \mathrm{E}+04$ & $2.03 \mathrm{E}+04$ & $2.03 \mathrm{E}+04$ & $2.03 E+04$ & 29.59 & 0.1 & 100 \\
\hline 5.2 & $1.94 \mathrm{E}+04$ & $1.93 \mathrm{E}+04$ & $1.93 \mathrm{E}+04$ & $1.93 \mathrm{E}+04$ & $1.93 E+04$ & 58.37 & 0.3 & 100 \\
\hline 7.1 & $2.00 \mathrm{E}+04$ & $1.98 \mathrm{E}+04$ & $1.97 \mathrm{E}+04$ & $1.97 \mathrm{E}+04$ & $1.98 E+04$ & 135.42 & 0.7 & 100 \\
\hline 7.2 & $2.01 \mathrm{E}+04$ & $2.00 \mathrm{E}+04$ & $2.01 \mathrm{E}+04$ & $2.01 \mathrm{E}+04$ & $2.00 E+04$ & 71.93 & 0.4 & 100 \\
\hline 8.1 & $1.97 \mathrm{E}+04$ & $1.97 \mathrm{E}+04$ & $1.96 \mathrm{E}+04$ & $1.97 \mathrm{E}+04$ & $1.97 E+04$ & 32.48 & 0.2 & 100 \\
\hline 10.1 & $2.04 \mathrm{E}+04$ & $2.02 \mathrm{E}+04$ & $2.02 \mathrm{E}+04$ & $2.01 \mathrm{E}+04$ & $2.02 E+04$ & 119.00 & 0.6 & 100 \\
\hline 11.1 & $2.03 \mathrm{E}+04$ & $1.99 \mathrm{E}+04$ & $1.98 \mathrm{E}+04$ & $1.98 \mathrm{E}+04$ & $2.00 E+04$ & 213.14 & 1.1 & 100 \\
\hline 12.1 & $2.01 \mathrm{E}+04$ & $2.00 \mathrm{E}+04$ & $1.99 \mathrm{E}+04$ & $2.00 \mathrm{E}+04$ & $2.00 E+04$ & 52.51 & 0.3 & 100 \\
\hline 12.2 & $2.00 \mathrm{E}+04$ & $1.98 \mathrm{E}+04$ & $1.97 \mathrm{E}+04$ & $1.97 \mathrm{E}+04$ & $1.98 E+04$ & 158.34 & 0.8 & 100 \\
\hline 12.3 & $2.00 \mathrm{E}+04$ & $1.98 \mathrm{E}+04$ & $1.99 \mathrm{E}+04$ & $1.97 \mathrm{E}+04$ & $1.99 E+04$ & 122.91 & 0.6 & 100 \\
\hline SRM 927d Control & $1.74 \mathrm{E}+04$ & $1.75 \mathrm{E}+04$ & $1.72 \mathrm{E}+04$ & $1.73 \mathrm{E}+04$ & $1.74 E+04$ & 115.52 & 0.7 & 100 \\
\hline \multicolumn{9}{|c|}{ Inter-Vial Repeatability } \\
\hline Avg. Peak Area (mAU) & $1.99 E+04$ & $1.98 E+04$ & $1.98 E+04$ & $1.98 E+04$ & & & & \\
\hline Stdev & 648.47 & 640.45 & 683.08 & 665.33 & & & & \\
\hline$\% C V$ & 3.3 & 3.2 & 3.4 & 3.4 & & & & \\
\hline
\end{tabular}


Table C8. SRM 2925 LC-UV (214 nm) results for Stability Study.

\begin{tabular}{|c|c|c|c|c|c|c|c|c|c|c|}
\hline \multirow[b]{2}{*}{$\begin{array}{l}\text { SRM } 2925 \text { Stability Sample } \\
\text { Name }\end{array}$} & \multicolumn{4}{|c|}{ LC-UV Peak Area Measurements } & \multicolumn{3}{|c|}{ Intra-Vial Repeatability } & \multicolumn{3}{|c|}{ Inter-Vial Repeatability } \\
\hline & $\begin{array}{r}\text { Technical } \\
\text { Replicate } 1\end{array}$ & $\begin{array}{c}\text { Technical } \\
\text { Replicate } 2\end{array}$ & $\begin{array}{c}\text { Technical } \\
\text { Replicate } 3\end{array}$ & $\begin{array}{r}\text { Technical } \\
\text { Replicate } 4\end{array}$ & $\begin{array}{l}\text { Avg. Peak Area } \\
(\mathrm{mAU})\end{array}$ & Stdev & $\% \mathrm{CV}$ & $\begin{array}{l}\text { Avg. Peak Area } \\
\text { (mAU) }\end{array}$ & Stdev & $\% \mathrm{CV}$ \\
\hline 1M -80.1 & $2.96 \mathrm{E}+04$ & $2.86 \mathrm{E}+04$ & $2.81 \mathrm{E}+04$ & $2=2.81 \mathrm{E}+04$ & $2.86 E+04$ & $\begin{array}{lll}700.06 \\
\end{array}$ & 2.4 & $\begin{array}{l}2.32 E+04 \\
\end{array}$ & $4 \quad 4006.43$ & 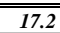 \\
\hline $1 \mathrm{M}-80.2$ & $2.15 \mathrm{E}+04$ & $2.10 \mathrm{E}+04$ & $2.08 \mathrm{E}+04$ & $2.08 \mathrm{E}+04$ & $2.10 E+04$ & 345.36 & 1.6 & & & \\
\hline $1 \mathrm{M}-80.3$ & $2.05 \mathrm{E}+04$ & $2.01 \mathrm{E}+04$ & $1.99 \mathrm{E}+04$ & $1.99 \mathrm{E}+04$ & $2.01 E+04$ & 293.61 & 1.5 & & & \\
\hline $1 \mathrm{M}-20.1$ & $2.40 \mathrm{E}+04$ & $2.31 \mathrm{E}+04$ & $2.31 \mathrm{E}+04$ & $2.31 \mathrm{E}+04$ & $2.33 E+04$ & 459.33 & 2.0 & $2.00 E+04$ & 3123.41 & 15.6 \\
\hline $1 \mathrm{M}-20.2$ & $1.46 \mathrm{E}+04$ & $1.75 \mathrm{E}+04$ & $1.66 \mathrm{E}+04$ & $1.62 \mathrm{E}+04$ & $1.62 E+04$ & 1236.89 & 7.6 & & & \\
\hline $1 \mathrm{M}-20.3$ & $2.09 \mathrm{E}+04$ & $2.02 \mathrm{E}+04$ & $2.02 \mathrm{E}+04$ & $2.00 \mathrm{E}+04$ & $2.03 E+04$ & 415.82 & 2.0 & & & \\
\hline 1M 5.1 & $2.05 E+04$ & $1.96 \mathrm{E}+04$ & $1.94 \mathrm{E}+04$ & $1.93 \mathrm{E}+04$ & $1.97 E+04$ & 545.62 & 2.8 & $1.96 E+04$ & 537.89 & 2.7 \\
\hline 1M 5.2 & $2.05 \mathrm{E}+04$ & $1.98 \mathrm{E}+04$ & $1.96 \mathrm{E}+04$ & $1.95 \mathrm{E}+04$ & $1.99 E+04$ & 414.97 & 2.1 & & & \\
\hline $1 \mathrm{M} 5.3$ & $2.00 \mathrm{E}+04$ & $1.93 \mathrm{E}+04$ & $1.88 \mathrm{E}+04$ & $1.89 \mathrm{E}+04$ & $1.92 E+04$ & 548.16 & 2.8 & & & \\
\hline 1M RT.1 & $2.18 \mathrm{E}+04$ & $2.03 \mathrm{E}+04$ & $2.00 \mathrm{E}+04$ & $1.99 \mathrm{E}+04$ & $2.05 E+04$ & 900.09 & 4.4 & $2.02 E+04$ & 858.92 & 4.2 \\
\hline 1M RT.2 & $2.10 \mathrm{E}+04$ & $1.98 \mathrm{E}+04$ & $1.91 \mathrm{E}+04$ & $1.89 \mathrm{E}+04$ & $1.97 E+04$ & 932.41 & 4.7 & $2.02 E+04$ & O50.02 & \\
\hline 1M RT.3 & $2.15 \mathrm{E}+04$ & $2.05 \mathrm{E}+04$ & $2.01 \mathrm{E}+04$ & $2.01 \mathrm{E}+04$ & $2.06 E+04$ & 645.19 & 3.1 & & & \\
\hline $1 \mathrm{M} 37.1$ & $1.91 \mathrm{E}+04$ & $2.08 \mathrm{E}+04$ & $1.61 \mathrm{E}+04$ & $2.07 \mathrm{E}+04$ & $1.92 E+04$ & 2182.86 & 11.4 & $2.12 E+04$ & 2132.07 & 10.1 \\
\hline 1M 37.2 & $2.32 \mathrm{E}+04$ & $2.18 \mathrm{E}+04$ & $2.09 \mathrm{E}+04$ & $2.03 \mathrm{E}+04$ & $2.16 E+04$ & 1266.96 & 5.9 & & & \\
\hline $1 \mathrm{M} 37.3$ & $2.42 \mathrm{E}+04$ & $2.30 \mathrm{E}+04$ & $2.25 \mathrm{E}+04$ & $2.14 \mathrm{E}+04$ & $2.28 E+04$ & 1161.55 & 5.1 & & & \\
\hline $2 \mathrm{~W}-20.1$ & $2.10 \mathrm{E}+04$ & $1.98 \mathrm{E}+04$ & $1.94 \mathrm{E}+04$ & $1.93 \mathrm{E}+04$ & $1.99 E+04$ & 775.65 & 3.9 & $1.94 E+04$ & 909.02 & 4.7 \\
\hline $2 \mathrm{~W}-20.2$ & $2.01 \mathrm{E}+04$ & $2.01 E+04$ & $1.99 \mathrm{E}+04$ & $1.97 \mathrm{E}+04$ & $1.99 E+04$ & 211.38 & 1.1 & & & \\
\hline $2 \mathrm{~W}-20.3$ & $1.94 \mathrm{E}+04$ & $1.84 \mathrm{E}+04$ & $1.81 \mathrm{E}+04$ & $1.79 \mathrm{E}+04$ & $1.84 E+04$ & 689.43 & 3.7 & & & \\
\hline 2W 5.1 & $1.93 \mathrm{E}+04$ & $1.95 \mathrm{E}+04$ & $1.94 \mathrm{E}+04$ & $1.94 \mathrm{E}+04$ & $1.94 E+04$ & 77.77 & 0.4 & $1.85 E+04$ & 1762.45 & 9.5 \\
\hline $2 \mathrm{~W} 5.2$ & $1.75 E+04$ & $1.63 \mathrm{E}+04$ & $1.58 \mathrm{E}+04$ & $1.56 \mathrm{E}+04$ & $1.63 E+04$ & 835.78 & 5.1 & & 170.45 & 0.5 \\
\hline $2 \mathrm{~W} 5.3$ & $2.12 \mathrm{E}+04$ & $1.97 \mathrm{E}+04$ & $1.94 \mathrm{E}+04$ & $1.93 \mathrm{E}+04$ & $1.99 E+04$ & 872.27 & 4.4 & & & \\
\hline 2W RT.1 & $1.96 \mathrm{E}+04$ & $1.80 \mathrm{E}+04$ & $1.73 \mathrm{E}+04$ & $1.74 \mathrm{E}+04$ & $1.81 E+04$ & 1047.26 & 5.8 & $1.94 E+04$ & 1164.57 & 6.0 \\
\hline 2W RT.2 & $2.10 \mathrm{E}+04$ & $2.01 \mathrm{E}+04$ & $1.97 \mathrm{E}+04$ & $1.97 \mathrm{E}+04$ & $2.01 E+04$ & 625.10 & 3.1 & & & \\
\hline 2W RT.3 & $2.03 \mathrm{E}+04$ & $1.98 \mathrm{E}+04$ & $1.99 \mathrm{E}+04$ & $1.99 \mathrm{E}+04$ & $2.00 E+04$ & 197.26 & 1.0 & & & \\
\hline $2 \mathrm{~W} 37.1$ & $2.27 \mathrm{E}+04$ & $2.12 \mathrm{E}+04$ & $2.16 \mathrm{E}+04$ & $2.09 \mathrm{E}+04$ & $2.16 E+04$ & 771.38 & 3.6 & $2.10 E+04$ & 778.15 & 3.7 \\
\hline $2 \mathrm{~W} 37.2$ & $2.15 \mathrm{E}+04$ & $2.02 \mathrm{E}+04$ & $2.03 \mathrm{E}+04$ & $1.98 \mathrm{E}+04$ & $2.04 E+04$ & 724.35 & 3.5 & & & \\
\hline $2 \mathrm{~W} 37.3$ & $2.15 \mathrm{E}+04$ & $2.13 \mathrm{E}+04$ & $2.07 \mathrm{E}+04$ & $2.05 \mathrm{E}+04$ & $2.10 E+04$ & 454.09 & 2.2 & & & \\
\hline $1 \mathrm{~W}-20.1$ & $1.98 \mathrm{E}+04$ & $1.99 \mathrm{E}+04$ & $1.98 \mathrm{E}+04$ & $2.00 \mathrm{E}+04$ & $1.99 E+04$ & 84.92 & 0.4 & $1.88 E+04$ & 1684.79 & 9.0 \\
\hline $1 \mathrm{~W}-20.2$ & $1.74 \mathrm{E}+04$ & $1.65 \mathrm{E}+04$ & $1.62 \mathrm{E}+04$ & $1.61 \mathrm{E}+04$ & $1.65 E+04$ & 608.08 & 3.7 & & & \\
\hline $1 \mathrm{~W}-20.3$ & $2.01 \mathrm{E}+04$ & $1.98 \mathrm{E}+04$ & $1.99 \mathrm{E}+04$ & $1.98 \mathrm{E}+04$ & $1.99 E+04$ & 151.49 & 0.8 & & & \\
\hline 1W 5.1 & $2.00 \mathrm{E}+04$ & $1.93 \mathrm{E}+04$ & $1.92 \mathrm{E}+04$ & $1.91 \mathrm{E}+04$ & $1.94 E+04$ & 418.44 & 2.2 & $1.99 E+04$ & 737.74 & 3.7 \\
\hline 1W 5.2 & $2.05 \mathrm{E}+04$ & $1.97 \mathrm{E}+04$ & $1.95 \mathrm{E}+04$ & $1.94 \mathrm{E}+04$ & $1.98 E+04$ & 517.46 & 2.6 & & & \\
\hline 1W 5.3 & $2.17 \mathrm{E}+04$ & $2.03 \mathrm{E}+04$ & $2.01 \mathrm{E}+04$ & $2.01 \mathrm{E}+04$ & $2.05 E+04$ & 800.10 & 3.9 & & & \\
\hline 1W RT.1 & $2.14 \mathrm{E}+04$ & $2.04 \mathrm{E}+04$ & $2.02 \mathrm{E}+04$ & $2.02 \mathrm{E}+04$ & $2.06 E+04$ & 583.07 & 2.8 & $2.02 E+04$ & 612.62 & 3.0 \\
\hline 1W RT.2 & $2.01 \mathrm{E}+04$ & $2.00 \mathrm{E}+04$ & $2.03 \mathrm{E}+04$ & $2.02 \mathrm{E}+04$ & $2.02 E+04$ & 118.60 & 0.6 & & & \\
\hline 1W RT.3 & $2.10 \mathrm{E}+04$ & $1.98 \mathrm{E}+04$ & $1.94 \mathrm{E}+04$ & $1.92 \mathrm{E}+04$ & $1.99 E+04$ & 824.01 & 4.2 & & & \\
\hline 1W 37.1 & $2.29 \mathrm{E}+04$ & $2.23 \mathrm{E}+04$ & $2.19 \mathrm{E}+04$ & $2.13 \mathrm{E}+04$ & $2.21 E+04$ & 651.18 & 2.9 & $2.13 E+04$ & 1427.98 & 6.7 \\
\hline 1W 37.2 & $2.09 \mathrm{E}+04$ & $1.94 \mathrm{E}+04$ & $1.99 \mathrm{E}+04$ & $1.93 \mathrm{E}+04$ & $1.99 E+04$ & 754.45 & 3.8 & & & \\
\hline 1W 37.3 & $2.40 \mathrm{E}+04$ & $2.20 \mathrm{E}+04$ & $2.08 \mathrm{E}+04$ & $2.04 \mathrm{E}+04$ & $2.18 E+04$ & 1602.85 & 7.3 & & & \\
\hline $3 \mathrm{D}-20.1$ & $1.88 \mathrm{E}+04$ & $1.89 \mathrm{E}+04$ & $1.90 \mathrm{E}+04$ & $1.88 \mathrm{E}+04$ & $1.89 E+04$ & 106.16 & 0.6 & $1.71 E+04$ & 2403.08 & 14.0 \\
\hline $3 D-20.2$ & $1.57 \mathrm{E}+04$ & $1.44 \mathrm{E}+04$ & $1.42 \mathrm{E}+04$ & $1.39 \mathrm{E}+04$ & $1.45 E+04$ & 773.68 & 5.3 & & & \\
\hline $3 D-20.3$ & $2.16 \mathrm{E}+04$ & $1.79 \mathrm{E}+04$ & $1.65 \mathrm{E}+04$ & $1.60 \mathrm{E}+04$ & $1.80 E+04$ & 2525.11 & 14.0 & & & \\
\hline 3D 5.1 & $1.93 \mathrm{E}+04$ & $1.84 \mathrm{E}+04$ & $1.79 \mathrm{E}+04$ & $1.77 \mathrm{E}+04$ & $1.83 E+04$ & 692.62 & 3.8 & $1.84 E+04$ & 1376.64 & 7.5 \\
\hline 3D 5.2 & $1.85 \mathrm{E}+04$ & $1.71 \mathrm{E}+04$ & $1.66 \mathrm{E}+04$ & $1.65 \mathrm{E}+04$ & $1.72 E+04$ & 915.96 & 5.3 & & & \\
\hline 3D 5.3 & $2.10 \mathrm{E}+04$ & $2.02 \mathrm{E}+04$ & $1.94 \mathrm{E}+04$ & $1.87 \mathrm{E}+04$ & $1.98 E+04$ & 975.63 & 4.9 & & & \\
\hline 3D RT.1 & $1.71 E+04$ & $1.64 \mathrm{E}+04$ & $1.64 \mathrm{E}+04$ & $1.65 E+04$ & $1.66 E+04$ & 365.68 & 2.2 & $1.76 E+04$ & 1540.89 & 8.8 \\
\hline 3D RT.2 & $1.84 \mathrm{E}+04$ & $1.69 \mathrm{E}+04$ & $1.64 \mathrm{E}+04$ & $1.65 \mathrm{E}+04$ & $1.70 E+04$ & 891.93 & 5.2 & & & \\
\hline 3D RT.3 & $1.69 \mathrm{E}+04$ & $2.08 \mathrm{E}+04$ & $1.97 \mathrm{E}+04$ & $1.93 \mathrm{E}+04$ & $1.92 E+04$ & 1650.06 & 8.2 & & & \\
\hline 3D 37.1 & $2.15 \mathrm{E}+04$ & $2.05 \mathrm{E}+04$ & $2.00 \mathrm{E}+04$ & $2.01 \mathrm{E}+04$ & $2.05 E+04$ & 669.97 & 3.3 & $2.06 E+04$ & 592.92 & 2.9 \\
\hline 3D 37.2 & $2.04 \mathrm{E}+04$ & $2.00 \mathrm{E}+04$ & $2.01 \mathrm{E}+04$ & $2.02 E+04$ & $2.02 E+04$ & 187.71 & 0.9 & & & \\
\hline 3 D 37.3 & $2.18 \mathrm{E}+04$ & $2.07 \mathrm{E}+04$ & $2.05 \mathrm{E}+04$ & $2.09 \mathrm{E}+04$ & $\begin{array}{l}2.02 E+04 \\
2.10 E+04\end{array}$ & 584.80 & 2.9 & & & \\
\hline SRM 927d Control 1 & $1.55 \mathrm{E}+04$ & $1.54 \mathrm{E}+04$ & $1.54 \mathrm{E}+04$ & $1.53 \mathrm{E}+04$ & $1.54 E+04$ & 86.88 & 0.6 & $1.59 E+04$ & 423.27 & 2.7 \\
\hline SRM 927d Control 2 & $1.62 E+04$ & $1.63 \mathrm{E}+04$ & $1.63 \mathrm{E}+04$ & $1.63 \mathrm{E}+04$ & $1.63 E+04$ & 49.53 & 0.3 & & & \\
\hline SRM 927d Control 3 & $1.64 \mathrm{E}+04$ & $1.62 \mathrm{E}+04$ & $1.63 \mathrm{E}+04$ & $1.56 \mathrm{E}+04$ & $1.61 E+04$ & 347.55 & 2.2 & & & \\
\hline
\end{tabular}




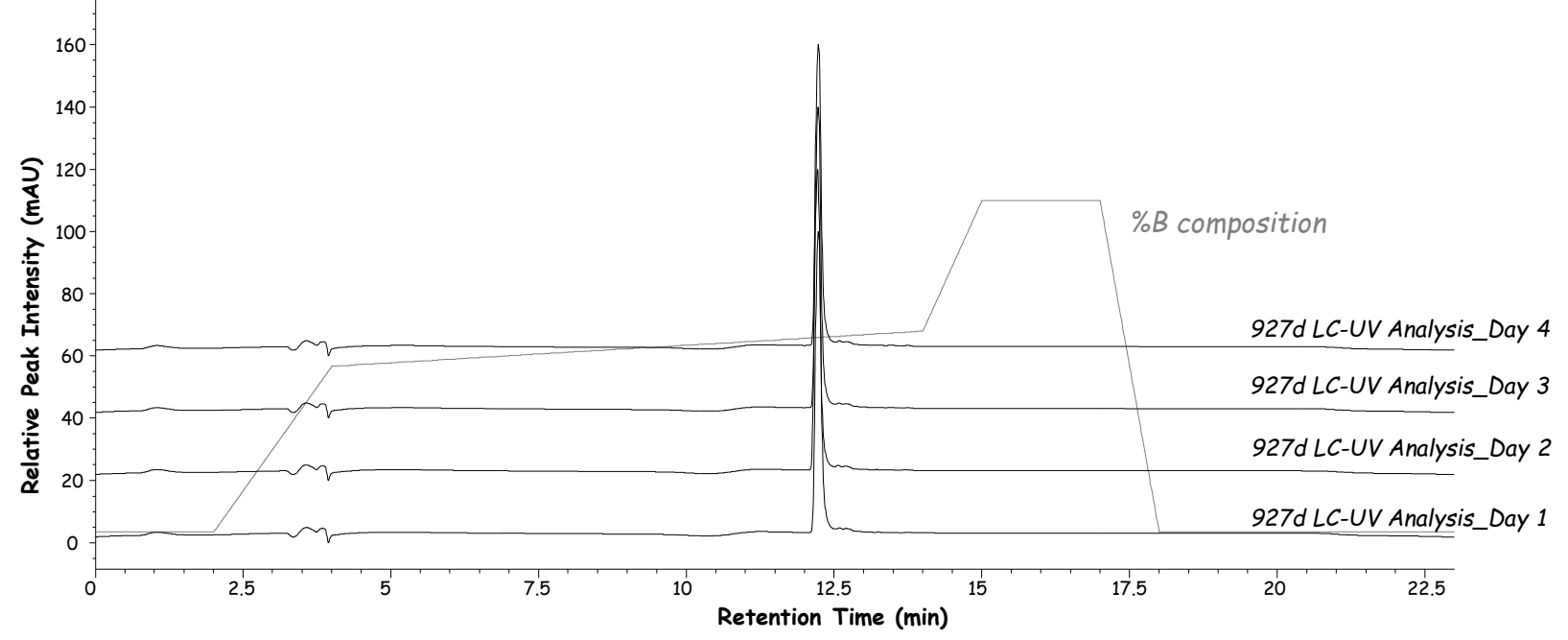

Figure C1. Instrument precision UV chromatogram of SRM 927d Control (Day 1-4). 


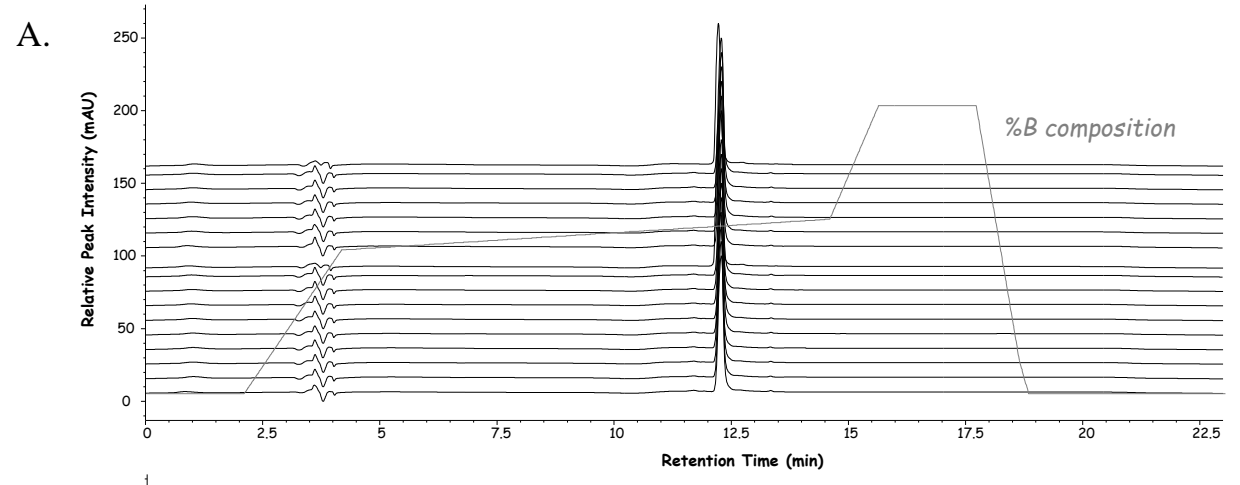

B.

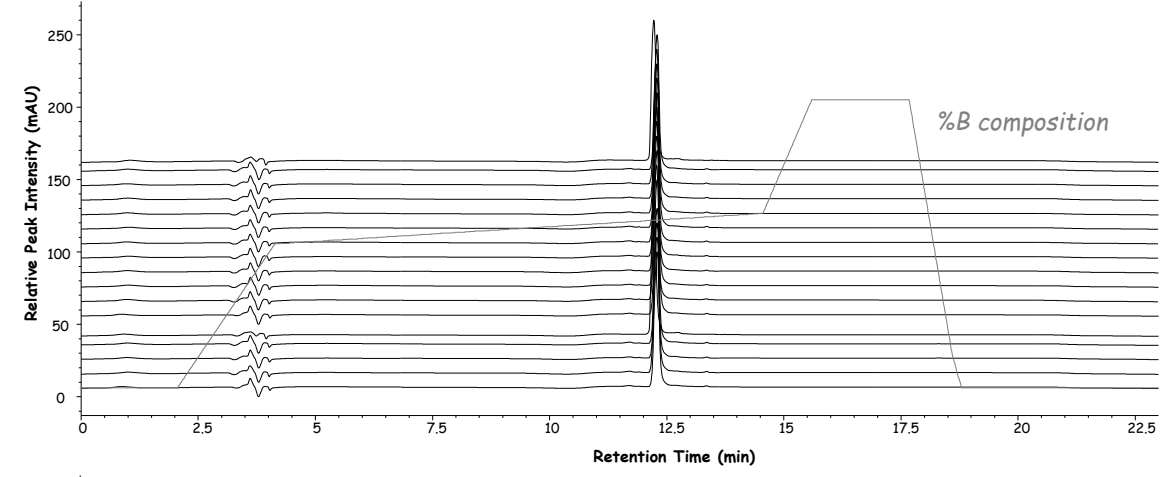

C.
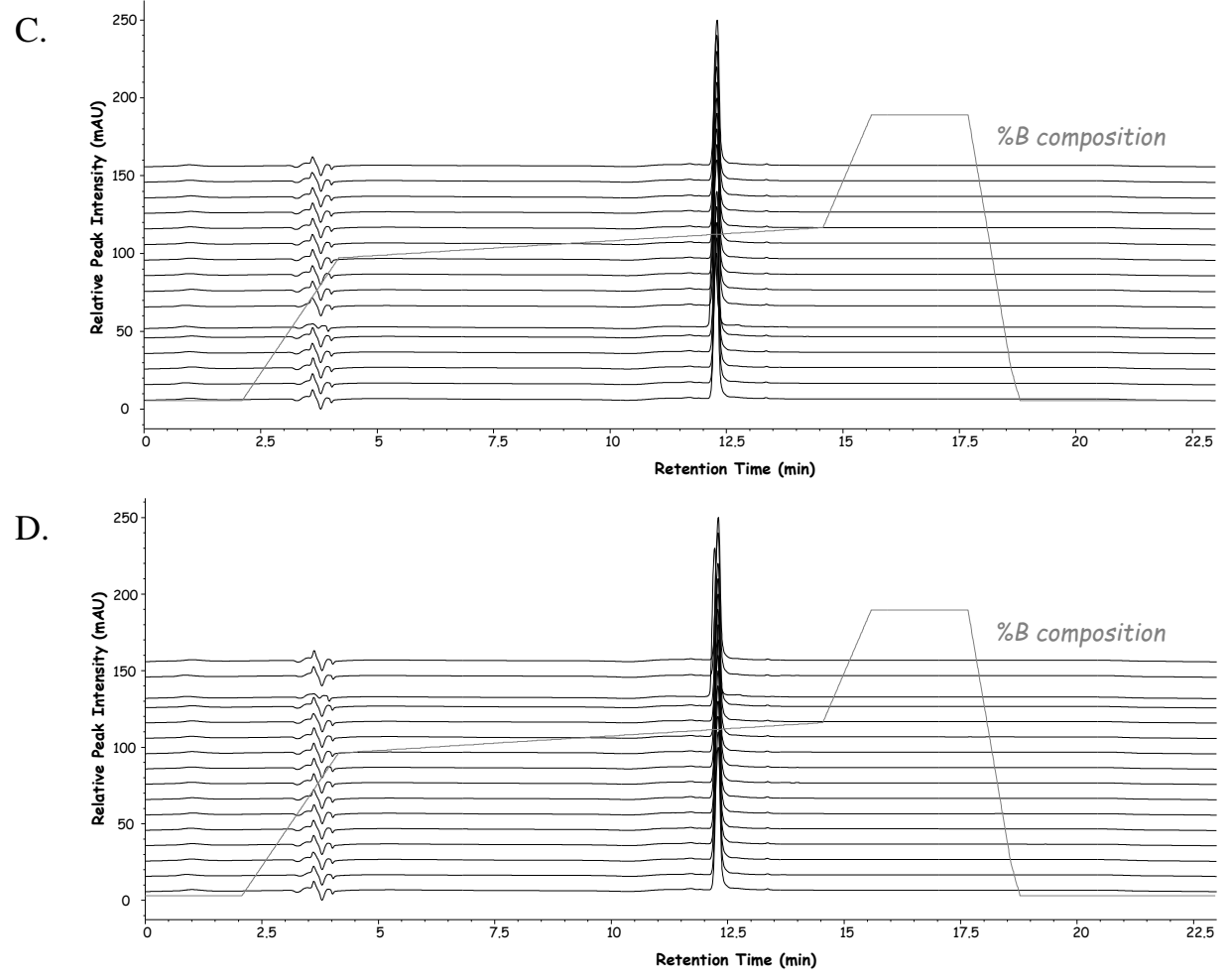

Figure C2. SRM 2925 Homogeneity Study UV Chromatogram: (A) Run 1, (B) Run 2, (C) Run 3 and (D) Run 4 

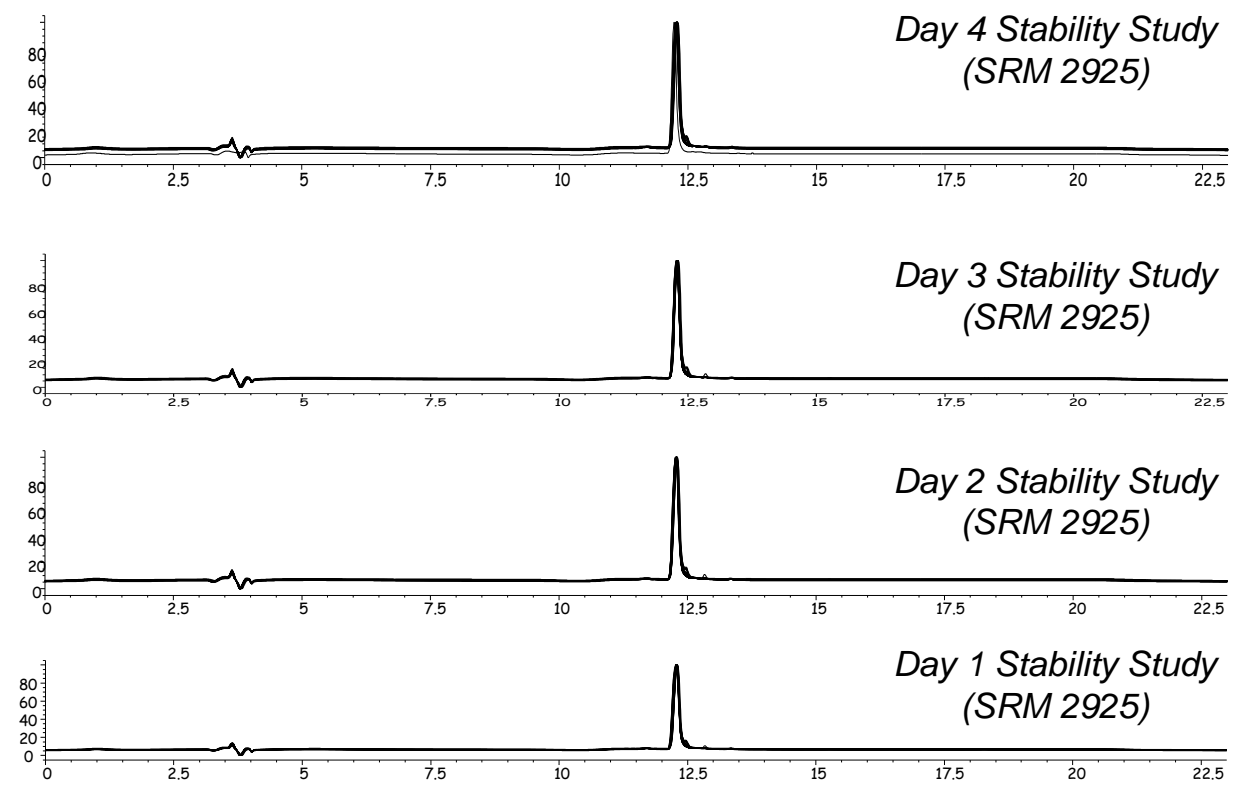

Figure C3. SRM 2925 Stability Study UV Chromatogram: Run 1 (A), Run 2 (B), Run 3 (C), Run 4 (D). 


\section{APPENDIX D: Certification of Total Protein content in SRM 2925}

\section{INTRODUCTION}

This report describes the certification of recombinant HSA concentration in SRM 2925 via amino acid analysis. An isotope dilution-liquid chromatography tandem mass spectrometry (ID-LC-MS/MS) method based on multiple reaction monitoring (MRM) was used for absolute quantification of the following amino acids: Alanine (Ala), Phenylalanine (Phe), and Arginine (Arg).

\section{EXPERIMENTAL}

SRM 2925 Recombinant Human Serum Albumin (Primary Reference Calibrator for Urine Albumin) (Frozen) is a solution of recombinant human albumin serum (HSA) in $50 \mathrm{mmol} / \mathrm{L}$ ammonium bicarbonate in water. Each vial of SRM 2925 contains approximately $0.50 \mathrm{~mL}$ of solution, which is stored frozen at approximately $-80{ }^{\circ} \mathrm{C}$. The material was obtained from Albumin Bioscience, a business unit of Albumin Therapeutics, LLC, (Huntsville, AL, USA) and the recombinant HSA was produced from a Pichia pastoris expression system. SRM 2925 was prepared at NIST by desalting the stock material ( $5 \%$ solution of phosphate buffer saline containing $4 \mathrm{mmol} / \mathrm{L}$ sodium caprylate and $4 \mathrm{mmol} / \mathrm{L}$ acetyltryptophan) via gel filtration chromatography (3,000 Da cutoff) to remove the storage buffer and diluting the desalted material in 50 $\mathrm{mmol} / \mathrm{L}$ ammonium bicarbonate in water.

Two vials of SRM 927e Bovine Serum Albumin (7\% Solution) were selected as quality control (QC) materials for quantification of SRM 2925. The concentration of SRM 927e $(67.38 \mathrm{~g} / \mathrm{L} \pm 1.38 \mathrm{~g} / \mathrm{L})$ is approximately 75fold higher than SRM 2925; therefore, each SRM 927e stock was diluted approximately 100 -fold in $0.1 \mathrm{~mol} / \mathrm{L}$ $\mathrm{HCl}$ in water ( $\sim 0.70 \mathrm{~g} / \mathrm{L})$ for use as a QC material for this study. The diluted SRM 927e solutions were stored at $4{ }^{\circ} \mathrm{C}$ until use. Gravimetric measurements and calculations of the diluted SRM 927e solutions are detailed in Table D1.

The amino acid certified reference materials (CRMs) used to prepare the calibrators for amino acid analysis were obtained from the National Metrology Institute of Japan (NMIJ) and were purchased through Wako Chemicals USA (Richmond, VA). The NMIJ amino acid CRMs used were CRM 6011-a (Alanine), CRM 6017-b (Arginine), and CRM 6014-a (Phenylalanine). The isotopically-labelled amino acids used as internal standards were obtained from Cambridge Isotope Laboratories (CIL; Andover, MA). The amino acid CRMs and isotopically-labeled amino acid internal standards (IS) used in this study are listed in Table D2A and D2B, respectively.

Sample Preparation. To prepare calibration solutions for amino acid analysis, stock and working solutions were prepared for each of the isotopically-labeled IS and unlabeled amino acids. Three sets of the stock and working solutions were prepared for each amino acid analysis set (Set 1, 2, and 3). The gravimetric measurements of the stock and working solutions for the unlabeled amino acids are listed in Table D3 and D4, respectively. The gravimetric measurements of the stock and workings solutions for the isotopicallylabeled amino acids are listed in Table D5 and D6, respectively. The diluent for both the stock and working solutions was $0.1 \mathrm{~mol} / \mathrm{L} \mathrm{HCl}$ in water. The working solutions for the isotopically-labeled IS and unlabeled amino acids were prepared from the stock solutions to a final concentration determined by the estimated molar concentration equivalence of each amino acid predicted by the know protein sequence of HSA (Table D7).

Independent calibration sets were prepared from the unique working stocks of the unlabeled and labeled amino acids for the three datasets (Set 1, 2, and 3). The unlabeled amino acid working solutions were added gravimetrically into $400-\mu \mathrm{L}$ flat bottom borosilicate glass inserts (Agilent 5181-3377, oven baked prior to use at $450^{\circ} \mathrm{C}$ for 8 hours), followed by addition of labeled amino acid working solutions. The calibration curves consisted of eight calibrants at $\approx 0.6,0.8,0.9,1.0,1.1,1.2,1.4$, and 1.5-fold the estimated analyte 
concentrations. The gravimetric measurements of the calibration blends for each set (Set 1, 2, and 3) are listed in Table D8. The calibrant sets were dried (Labconco CentriVap Complete) without heating and subjected to the hydrolysis procedure as described below in the "Vapor Phase Acid Hydrolysis" section.

Six vials of SRM 2925 for each set (total of 18 vials) were removed from $-80{ }^{\circ} \mathrm{C}$ storage and placed in $4{ }^{\circ} \mathrm{C}$ storage to thaw overnight prior to use. The vials were vortexed and equilibrated in the weigh room for at least one hour before diluting. Approximately $60 \mu \mathrm{L}$ of SRM 2925 was added gravimetrically to a glass autosampler vial insert followed by $60 \mu \mathrm{L}$ of the labeled amino acid working solution corresponding to each Sample Set (Set 1, 2, and 3). Gravimetric measurements of the SRM 2925 and QC (SRM 927e) samples are listed in Table D9. The samples were dried (Labconco CentriVap Complete) without heating and subjected to the hydrolysis procedure described below in the "Vapor Phase Acid Hydrolysis" section. The diluted SRM 927e solutions (QC materials) were treated the same as the SRM 2925 samples.

Vapor Phase Acid Hydrolysis. The dried samples (calibrants, SRM 2925 samples, and SRM 927e QC samples) were loaded into the Teflon sample disk (capacity of 16 vials) and then into a Teflon reactor liner. Prior to placing the loaded Teflon sample disk, approximately $2.0 \mathrm{~mL}$ of $6 \mathrm{~mol} / \mathrm{L} \mathrm{HCL}$ was added to the bottom of the vessel. The loaded Teflon reactor liner was added to a stainless steel hydrothermal synthesis reactor vessel and placed inside the Memmert UF55 oven. The vessel was heated for 24 hours at $120^{\circ} \mathrm{C}$ for hydrolysis of the samples (optimized reaction conditions determined during preliminary assessment). After hydrolysis, the loaded hydrothermal synthesis reactor vessel was removed from the oven and cooled on the bench for 1 hour prior to removing the Teflon sample disk. The glass inserts were removed from the Teflon sample disk and dried in a Labconco CentriVap Complete vacuum dryer without heat to remove residual $\mathrm{HCl}$. The dried samples were reconstituted directly in the glass inserts with $\approx 200 \mu \mathrm{L}$ of $0.1 \mathrm{~mol} / \mathrm{L}$ $\mathrm{HCl}$ in water (volumetric measurements) and stored at $5{ }^{\circ} \mathrm{C}$ until LC-MS analysis.

Instrumental method. Liquid chromatographic separation was performed using a SieLC Primesep 100 analytical column, $2.1 \mathrm{~mm}$ x $250 \mathrm{~mm}, 3 \mu \mathrm{m}$ particle size, $100 \AA$ pore size, (SieLC, Item\# 100-21.250.0310) at a flow rate of $200 \mu \mathrm{L} / \mathrm{min}$. An Agilent Infinity 1290 UPLC system was coupled in-line with an Agilent 6460 triple quadrupole mass spectrometer with a Jet Stream equipped standard micro-flow source. The "A" mobile phase used for amino acid separations was $0.05 \%$ (v/v) TFA in $80 \%$ (v/v) water, $20 \%$ (v/v) ACN and the "B" mobile phase consisted of $0.45 \%(\mathrm{v} / \mathrm{v})$ TFA in $80 \%(\mathrm{v} / \mathrm{v})$ water, $20 \%(\mathrm{v} / \mathrm{v})$ ACN. Details of the LC gradient is listed in Table D10. The column temperature was maintained at $15^{\circ} \mathrm{C}$ for all experiments and the autosampler plate was maintained at $5{ }^{\circ} \mathrm{C}$. Tandem MS was performed in positive mode using unit resolution for monitoring the precursor ion/product ion MRM transition. Table D11 outlines the MRM parameters for amino acid analysis. Source conditions were held constant for the entire run: source temperature $=300{ }^{\circ} \mathrm{C}$; gas flow $=6 \mathrm{~L} / \mathrm{min}$; nebulizer gas $=45 \mathrm{psi}$; sheath gas temperature $=250$ ${ }^{\circ} \mathrm{C}$; sheath gas flow $=11 \mathrm{~L} / \mathrm{min}$; capillary voltage $=4000 \mathrm{~V}$; nozzle voltage $=500 \mathrm{~V} ; \Delta \mathrm{EMV}=+350$.

Qualitative/Quantitative Method. Integration of chromatographic peaks was performed using Agilent MassHunter Qualitative Analysis software (Version B.09.00). All peak integrations were manually confirmed or corrected, as needed. Peak areas from MassHunter integration were imported to Microsoft Excel for further quantitative analysis.

The molar concentration (nmol/g) for the amino acids were converted to mass concentration (g/L) using the theoretical molar mass of mature, native HSA. The relative molecular mass was calculated from the atomic masses of the total number of carbon $\left({ }^{12} \mathrm{C}\right)$, nitrogen $\left({ }^{14} \mathrm{~N}\right)$, oxygen $\left({ }^{16} \mathrm{O}\right)$, sulfur $\left({ }^{32} \mathrm{~S}\right)$, and hydrogen $\left({ }^{1} \mathrm{H}\right)$ elements in each amino acid for the mature (residues 25-609) and native (including 17 disulfide linkages) amino acid sequence of HSA (see Figure D1 for amino acid sequence of HSA). As shown in Table D12, the relative molecular mass of HSA is $66,394.56 \mathrm{~g} / \mathrm{mol}$ based on the reported mature amino acid sequence and post-translational modifications (17 disulfide bonds). 
Preliminary Experiments. Prior to certification of SRM 2925, an optimization assessment of the hydrolysis reaction was conducted to determine the optimal temperature and reaction time for amino acid analysis of SRM 2925. Hydrolysis was evaluated at $140{ }^{\circ} \mathrm{C}$ and $120^{\circ} \mathrm{C}$ for 24 hours and 48 hours. IS and calibration was performed as described above so that concentration was used as the end point. Each time-point and temperature combination were performed in a separate hydrolysis vessel and included a full set of calibrants. As illustrated in Figure D2, the optimal hydrolysis condition for SRM 2925 certification was $120{ }^{\circ} \mathrm{C}$ for 24 hours.

\section{RESULTS AND DISCUSSION}

Figure D3 shows the total ion chromatograms (TICs) of the LC-MS/MS analysis of an amino acid calibration blend (Figure D3A) and of one sample of SRM 2925 (Figure D3B). The calibration curves for Sample Set 1, 2, and 3 (Ala, Phe, Arg) are shown in Figure D4, 5, and 6, respectively. The concentration measurements for SRM 2925 and SRM 927e (QC) were validated by assessing the intra-/inter-amino acid precision and the within- and between-set precision.

To calculate the mass concentration value of recombinant HSA in SRM 2925, the average molar mass concentration for the three amino acids $(14.4 \mathrm{nmol} / \mathrm{g} \pm 0.3 \mathrm{nmol} / \mathrm{g})$ was used in the equation below and the mass concentration for recombinant HSA in SRM 2925 was determined to be $0.958 \mathrm{~g} / \mathrm{L} \pm 0.023 \mathrm{~g} / \mathrm{L}$ (reference value).

Mass Concentration Value of Recombinant HSA in SRM 2925 (g/L) = (molar concentration value (nmol/g)/ $\left.10^{6}\right)$ (molecular mass) (Density $(\mathrm{g} / \mathrm{mL})$ )

Mass Concentration Value of Recombinant HSA in SRM 2925 (g/L) $=\left(14.4 \mathrm{nmol} / \mathrm{g} / 10^{6}\right)(66394.56 \mathrm{~g} / \mathrm{mol})$ $(1.00016 \mathrm{~g} / \mathrm{mL})=0.958 \mathrm{~g} / \mathrm{L}$

\section{CONCLUSION}

SRM 2925 appears to be fit-for-purpose as a primary reference material for HSA measurements using mass spectrometry. The value-assignment of the mass concentration of recombinant HSA in SRM 2925 was performed with sufficient precision $(<2 \%)$ necessary for use of the material in the value-assignment of urine albumin (HSA) in candidate SRM 3666, the matrix-based secondary reference material for the urine albumin reference measurement system. The final mass concentration values for SRM 2925 and SRM 927e (QC material) are listed in Table D15 and D16, respectively. The overall mass concentration of recombinant HSA in SRM 2925, as determined by amino acid analysis using three (3) amino acids (Ala, Phe, and Arg), is $14.4 \mathrm{nmol} / \mathrm{g} \pm 0.3 \mathrm{nmol} / \mathrm{g}$ with a coefficient of variation (CV) value of $2.4 \%$. The measurements of the three (3) amino acids (Ala, Phe, and Arg) was performed with sufficient precision and the results are suitable for value-assignment of the molar concentration of recombinant HSA in SRM 2925. 
Table D1. Gravimetric measurements for dilutions of SRM 927e Quality Controls.

\begin{tabular}{|c|c|c|c|}
\hline QC \# & Mass of 927e (g) & Diluent (g) & Dilution Factor \\
\hline \hline QC-1 & 0.01086 & 0.99061 & 92.2 \\
\hline QC-2 & 0.01058 & 0.99145 & 94.7 \\
\hline
\end{tabular}

Table D2-A. Unlabeled amino acid CRMs for amino acid analysis.

\begin{tabular}{|c|c|c|c|}
\hline Amino Acid & Origin & NMIJ CRM \# & NMIJ CRM Lot \# \\
\hline \hline Alanine & NMIJ CRM & $6011-\mathrm{a}$ & 102 \\
\hline Arginine & NMIJ CRM & $6017-\mathrm{b}$ & 39 \\
\hline Phenylalanine & NMIJ CRM & $6014-\mathrm{a}$ & 144 \\
\hline
\end{tabular}

Table D2-B: Isotopically-labeled amino acids for amino acid analysis.

\begin{tabular}{|c|c|c|c|c|}
\hline Amino Acid & Origin & Product Number & Lot Number & PSO Number \\
\hline \hline L-ALANINE (1-13C, 99 \%; 15N, 98 \%) & Cambridge Isotopes & CNLM-6993-0.25 & PR-17139 & $17 \mathrm{E}-685$ \\
\hline L-ARGININE: HCL (15N4, 98 \%) & Cambridge Isotopes & NLM-396-0.1 & PR-22210 & $13 \mathrm{~J}-096$ \\
\hline L-PHENYLALANINE (13C9, 99 \%; 15N, 99 \%) & Cambridge Isotopes & CNLM-575-H-0.25 & PR-26728 & $16 \mathrm{G}-604$ \\
\hline
\end{tabular}

Table D3: Gravimetric measurements of amino acid stock solutions for unlabeled amino acid CRMs

\begin{tabular}{|c|c|c|c|c|c|c|c|}
\hline $\begin{array}{c}\text { Sample } \\
\text { Set }\end{array}$ & Amino Acid & $\begin{array}{c}\text { Mass of } \\
\text { Amino Acid } \\
\text { (g) }\end{array}$ & $\begin{array}{c}\text { Mass of Diluent } \\
(0.1 \mathrm{M} \mathrm{HCl})(\mathrm{g})\end{array}$ & $\begin{array}{c}\text { Concentration } \\
(\mathrm{mg} / \mathrm{g})\end{array}$ & $\begin{array}{c}\text { MW of } \\
\text { Amino Acid } \\
\text { (g/mol) }\end{array}$ & $\begin{array}{c}\text { Molarity } \\
(\mathrm{pmol} / \mu \mathrm{L})\end{array}$ & $\begin{array}{c}\begin{array}{c}\text { Final Stock Solution } \\
\text { Concentration } \\
\text { (pmol/g) }\end{array} \\
\end{array}$ \\
\hline \multirow[t]{3}{*}{ Set 1} & Alanine & 0.04776 & 4.99471 & 0.010 & 89.1 & 107318.9306 & 106302454 \\
\hline & Arginine & 0.00485 & 5.01805 & 0.001 & 174.2 & 5548.282983 & 5542926 \\
\hline & Phenylalanine & 0.04956 & 4.99343 & 0.010 & 165.2 & 60078.94373 & 59488518 \\
\hline \multirow[t]{3}{*}{ Set 2} & Alanine & 0.06008 & 5.00183 & 0.012 & 89.1 & 134810.3676 & 133210298 \\
\hline & Arginine & 0.00876 & 4.99235 & 0.002 & 174.2 & 10072.81669 & 10055173 \\
\hline & Phenylalanine & 0.03066 & 5.00366 & 0.006 & 165.2 & 37091.49309 & 36865599 \\
\hline \multirow[t]{3}{*}{ Set 3} & Alanine & 0.05504 & 4.99090 & "0.011 & 89.1 & 123771.8416 & 122421766 \\
\hline & Arginine & 0.00876 & 4.99235 & 0.002 & 174.2 & 10072.81669 & 10055173 \\
\hline & Phenylalanine & 0.02262 & 5.00211 & 0.005 & 165.2 & 27373.4363 & 27250208 \\
\hline
\end{tabular}

Table D4: Gravimetric measurements of unlabeled amino acid CRM working solutions

\begin{tabular}{|c|c|c|c|c|c|}
\hline $\begin{array}{c}\text { Sample } \\
\text { Set }\end{array}$ & Amino Acid & $\begin{array}{c}\text { Final Amino Acid Stock } \\
\text { Solution Concentration } \\
\text { (pmol/g) }\end{array}$ & $\begin{array}{c}\text { Amino Acid } \\
\text { Stock Solution } \\
\text { Mass (g) }\end{array}$ & $\begin{array}{c}\text { Working } \\
\text { Solution } \\
\text { Mass (g) }\end{array}$ & $\begin{array}{c}\text { Concentration of Amino } \\
\text { Acid in Working Solution } \\
\text { (pmol/g) }\end{array}$ \\
\hline \hline Set 1 & Alanine & 106302454 & 0.05988 & 7.88042 & $8.08 \mathrm{E}+05$ \\
\hline & Arginine & 5542926 & 0.43988 & 7.88042 & $3.09 \mathrm{E}+05$ \\
\hline & Phenylalanine & 59488518 & 0.05453 & 7.88042 & $4.12 \mathrm{E}+05$ \\
\hline \hline Set 2 & Alanine & 133210298 & 0.04767 & 7.98642 & $7.95 \mathrm{E}+05$ \\
\hline & Arginine & 10055173 & 0.24386 & 7.98642 & $3.07 \mathrm{E}+05$ \\
\hline \hline Set 3 & Phenylalanine & 36865599 & 0.08469 & 7.98642 & $3.91 \mathrm{E}+05$ \\
\hline & Alanine & 122421766 & 0.05120 & 7.89039 & $7.94 \mathrm{E}+05$ \\
\hline & Arginine & 10055173 & 0.24387 & 7.89039 & $3.11 \mathrm{E}+05$ \\
\hline
\end{tabular}


Table D5: Gravimetric measurements of amino acid stock solutions for isotopically-labeled amino acids

\begin{tabular}{|c|c|c|c|c|c|c|c|}
\hline Sample Set & Amino Acid & $\begin{array}{l}\text { Mass of Amino } \\
\text { Acid (g) }\end{array}$ & $\begin{array}{l}\text { Mass of Diluent (0.1 } \\
\mathrm{mol} / \mathrm{L} \mathrm{HCl})(\mathrm{g})\end{array}$ & $\begin{array}{c}\text { Concentration } \\
(\mathrm{mg} / \mathrm{g})\end{array}$ & $\begin{array}{l}\text { MW of Amino } \\
\text { Acid (g/mol) }\end{array}$ & $\begin{array}{l}\text { Molarity } \\
(\mathrm{pmol} / \mu \mathrm{L})\end{array}$ & $\begin{array}{c}\text { Final Stock Solution } \\
\begin{array}{c}\text { Concentration } \\
\text { (pmol/g) }\end{array} \\
\end{array}$ \\
\hline \multirow[t]{3}{*}{ Set 1} & $\begin{array}{c}\text { L-ALANINE (1-13C, } 99 \% \text {; } \\
\text { 15N, } 98 \%)\end{array}$ & 0.00945 & 5.02071 & 0.002 & 91.1 & 20623.66569 & 20622040 \\
\hline & $\begin{array}{c}\text { L-ARGININE:HCL (15N4, } \\
98 \%)\end{array}$ & 0.01045 & 4.98470 & 0.002 & 178.2 & 11743.2182 & 11739783 \\
\hline & $\begin{array}{l}\text { L-PHENYLALANINE } \\
(13 C 9,99 \% ; 15 N, 99 \%) \\
\end{array}$ & 0.00723 & 4.99399 & 0.001 & 175.2 & 8263.357213 & 8251411 \\
\hline \multirow[t]{3}{*}{ Set 2} & $\begin{array}{c}\text { L-ALANINE (1-13C, } 99 \% \text {; } \\
\text { 15N, } 98 \%)\end{array}$ & 0.00944 & 5.02622 & 0.002 & 91.1 & 20579.25691 & 20577718 \\
\hline & $\begin{array}{c}\text { L-ARGININE:HCL (15N4, } \\
98 \%)\end{array}$ & 0.00761 & 5.02021 & 0.002 & 178.2 & 8491.269758 & 8493706 \\
\hline & $\begin{array}{c}\text { L-PHENYLALANINE } \\
(13 \mathrm{C} 9,99 \% ; 15 N, 99 \%) \\
\end{array}$ & 0.00858 & 5.03215 & 0.002 & 175.2 & 9731.944147 & 9715379 \\
\hline \multirow[t]{3}{*}{ Set 3} & $\begin{array}{c}\text { L-ALANINE (1-13C, } 99 \% \text {; } \\
\text { 15N, } 98 \%)\end{array}$ & 0.00755 & 5.02687 & 0.002 & 91.1 & 16481.67157 & 16486593 \\
\hline & $\begin{array}{c}\text { L-ARGININE:HCL (15N4, } \\
98 \%)\end{array}$ & 0.00920 & 5.02952 & 0.002 & 178.2 & 10265.17347 & 10264873 \\
\hline & $\begin{array}{c}\text { L-PHENYLALANINE } \\
(13 C 9,99 \% ; 15 N, 99 \%)\end{array}$ & 0.01184 & 5.02736 & 0.002 & 175.2 & 13474.15795 & 13442425 \\
\hline
\end{tabular}

Table D6: Gravimetric measurements of isotopically-labeled amino acids working solutions

\begin{tabular}{|c|c|c|c|c|c|}
\hline $\begin{array}{c}\text { Sample } \\
\text { Set }\end{array}$ & Amino Acid & $\begin{array}{c}\text { Final Amino Acid Stock } \\
\text { Solution Concentration } \\
(\mathbf{p m o l} / \mathrm{g})\end{array}$ & $\begin{array}{l}\text { Amino Acid Stock } \\
\text { Solution Mass (g) }\end{array}$ & $\begin{array}{l}\text { Working Solution } \\
\text { Mass (g) }\end{array}$ & $\begin{array}{l}\text { Concentration of Amino Acid in } \\
\text { Working Solution (pmol/g) }\end{array}$ \\
\hline \multirow[t]{2}{*}{ Set 1} & $\begin{array}{c}\text { L-ALANINE (1-13C, } 99 \text { \%; 15N, } \\
98 \%)\end{array}$ & 20622040 & 0.31238 & 7.98842 & $8.06 \mathrm{E}+05$ \\
\hline & $\begin{array}{c}\text { L-PHENYLALANINE (13C9, } 99 \\
\% ; 15 N, 99 \%)\end{array}$ & 8251411 & 0.38006 & 7.98842 & $3.93 \mathrm{E}+05$ \\
\hline Set 2 & $\begin{array}{c}\text { L-ALANINE (1-13C, 99\%; 15N, } \\
98 \%)\end{array}$ & 20577718 & 0.31195 & 7.90227 & $8.12 \mathrm{E}+05$ \\
\hline \multirow[t]{3}{*}{ Set 3} & $\begin{array}{c}\text { L-ALANINE (1-13C, } 99 \% \text { \% 15N, } \\
98 \%)\end{array}$ & 16486593 & 0.39030 & 7.9232 & $8.12 \mathrm{E}+05$ \\
\hline & L-ARGININE:HCL (15N4, $98 \%)$ & 10264873 & 0.29407 & 7.9232 & $3.81 \mathrm{E}+05$ \\
\hline & $\begin{array}{c}\text { L-PHENYLALANINE (13C9, } 99 \\
\% ; 15 \mathrm{~N}, 99 \%)\end{array}$ & 13442425 & 0.23368 & 7.9232 & $3.96 \mathrm{E}+05$ \\
\hline
\end{tabular}


Table D7: Molar relationship between intact HSA protein and its constituent amino acids

\begin{tabular}{|c|c|}
\hline Amino Acid & $\begin{array}{c}\text { pmol of } \\
\text { AA/pmol HSA }\end{array}$ \\
\hline \hline Alanine & 62 \\
\hline Arginine & 24 \\
\hline Phenylalanine & 31 \\
\hline
\end{tabular}

Table D8: Gravimetric measurements of unlabeled and labeled (Internal Standard) working solutions to create calibration solutions for each set

\begin{tabular}{|c|c|c|c|c|c|c|c|}
\hline $\begin{array}{c}\text { Sample } \\
\text { Set } \\
\end{array}$ & $\begin{array}{c}\begin{array}{c}\text { Calibration } \\
\text { Level }\end{array} \\
\end{array}$ & $\begin{array}{l}\text { Expected Unlabeled: } \\
\text { Labeled (IS) Ratio } \\
\end{array}$ & $\begin{array}{c}\text { Unlabeled Working } \\
\text { Solution Volume }(\mu \mathrm{L}) \\
\end{array}$ & $\begin{array}{c}\text { Unlabeled Working } \\
\text { Solution Mass (g) } \\
\end{array}$ & $\begin{array}{l}\text { Labeled (IS) Working } \\
\text { Solution Volume }(\mu \mathrm{L}) \\
\end{array}$ & $\begin{array}{c}\text { Labeled (IS) Working } \\
\text { Solution Mass (g) } \\
\end{array}$ & $\begin{array}{l}\text { Actual Unlabeled: } \\
\text { Labeled (IS) Ratio } \\
\end{array}$ \\
\hline \multirow[t]{8}{*}{ Set 1} & 1.1 & 0.60 & 36 & 0.03762 & 60 & 0.05852 & 0.64 \\
\hline & 2.1 & 0.80 & 48 & 0.04852 & 60 & 0.05959 & 0.81 \\
\hline & 3.1 & 0.90 & 54 & 0.05159 & 60 & 0.05249 & 0.98 \\
\hline & 4.1 & 1.00 & 60 & 0.05923 & 60 & 0.05978 & 0.99 \\
\hline & 5.1 & 1.10 & 66 & 0.06477 & 60 & 0.05919 & 1.09 \\
\hline & 6.1 & 1.20 & 72 & 0.07135 & 60 & 0.05980 & 1.19 \\
\hline & 7.1 & 1.40 & 84 & 0.08316 & 60 & 0.05695 & 1.46 \\
\hline & 8.1 & 1.50 & 90 & 0.08948 & 60 & 0.06013 & 1.49 \\
\hline \multirow[t]{8}{*}{ Set 2} & 1.2 & 0.60 & 36 & 0.03631 & 60 & 0.06049 & 0.60 \\
\hline & 2.2 & 0.80 & 48 & 0.04731 & 60 & 0.05918 & 0.80 \\
\hline & 3.2 & 0.90 & 54 & 0.05402 & 60 & 0.05926 & 0.91 \\
\hline & 4.2 & 1.00 & 60 & 0.05869 & 60 & 0.05926 & 0.99 \\
\hline & 5.2 & 1.10 & 66 & 0.06604 & 60 & 0.06047 & 1.09 \\
\hline & 6.2 & 1.20 & 72 & 0.07190 & 60 & 0.05953 & 1.21 \\
\hline & 7.2 & 1.40 & 84 & 0.08140 & 60 & 0.06006 & 1.36 \\
\hline & 8.2 & 1.50 & 90 & 0.08863 & 60 & 0.06036 & 1.47 \\
\hline \multirow[t]{8}{*}{ Set 3} & 1.3 & 0.60 & 36 & 0.03644 & 60 & 0.06046 & 0.60 \\
\hline & 2.3 & 0.80 & 48 & 0.04792 & 60 & 0.05624 & 0.85 \\
\hline & 3.3 & 0.90 & 54 & 0.05448 & 60 & 0.05982 & 0.91 \\
\hline & 4.3 & 1.00 & 60 & 0.05882 & 60 & 0.05940 & 0.99 \\
\hline & 5.3 & 1.10 & 66 & 0.06475 & 60 & 0.05998 & 1.08 \\
\hline & 6.3 & 1.20 & 72 & 0.07216 & 60 & 0.06079 & 1.19 \\
\hline & 7.3 & 1.40 & 84 & 0.08392 & 60 & 0.06037 & 1.39 \\
\hline & 8.3 & 1.50 & 90 & 0.08972 & 60 & 0.05923 & 1.51 \\
\hline
\end{tabular}


Table D9: Gravimetric measurements of SRM 2925 and SRM 927e (QCs) samples for each sample set

\begin{tabular}{|c|c|c|c|c|c|c|c|}
\hline Sample Set & Calibration Level & $\begin{array}{l}\text { Expected Unlabeled: } \\
\text { Labeled (IS) Ratio }\end{array}$ & $\begin{array}{l}\text { Unlabeled Working } \\
\text { Solution Volume }(\mu \mathrm{L})\end{array}$ & $\begin{array}{l}\text { Unlabeled Working } \\
\text { Solution Mass (g) }\end{array}$ & $\begin{array}{l}\text { Labeled (IS) Working } \\
\text { Solution Volume }(\mu \mathrm{L})\end{array}$ & $\begin{array}{l}\text { Labeled (IS) Working } \\
\text { Solution Mass (g) }\end{array}$ & $\begin{array}{c}\text { Actual } \\
\text { Unlabeled:Labeled } \\
\text { (IS) Ratio } \\
\end{array}$ \\
\hline \multirow{8}{*}{ 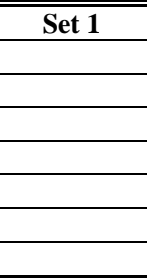 } & SRM 29251.1 & 1.00 & 60 & 0.05986 & 60 & 0.05269 & 1.14 \\
\hline & SRM 29251.2 & 1.00 & 60 & 0.05922 & 60 & 0.06004 & 0.99 \\
\hline & SRM 29251.3 & 1.00 & 60 & 0.05902 & 60 & 0.06066 & 0.97 \\
\hline & SRM 29251.4 & 1.00 & 60 & 0.05722 & 60 & 0.05596 & 1.02 \\
\hline & SRM 29251.5 & 1.00 & 60 & 0.05918 & 60 & 0.05962 & 0.99 \\
\hline & SRM 29251.6 & 1.00 & 60 & 0.06032 & 60 & 0.05972 & 1.01 \\
\hline & SRM 927e 1.1 & 1.00 & 60 & 0.05932 & 60 & 0.05900 & 1.01 \\
\hline & SRM 927e 1.2 & 1.00 & 60 & 0.05972 & 60 & 0.06023 & 0.99 \\
\hline \multirow[t]{8}{*}{ Set 2} & "SRM 29252.1 & 1.00 & 60 & 0.05657 & 60 & 0.06045 & 0.94 \\
\hline & SRM 29252.2 & 1.00 & 60 & 0.05886 & 60 & 0.06084 & 0.97 \\
\hline & SRM 29252.3 & 1.00 & 60 & 0.05956 & 60 & 0.05595 & 1.06 \\
\hline & SRM 29252.4 & 1.00 & 60 & 0.05755 & 60 & 0.06089 & 0.95 \\
\hline & SRM 29252.5 & 1.00 & 60 & 0.05838 & 60 & 0.05978 & 0.98 \\
\hline & SRM 29252.6 & 1.00 & 60 & 0.05855 & 60 & 0.05709 & 1.03 \\
\hline & SRM 927e 1.1 & 1.00 & 60 & 0.05500 & 60 & 0.06045 & 0.91 \\
\hline & SRM 927e 1.2 & 1.00 & 60 & 0.05993 & 60 & 0.06021 & 1.00 \\
\hline \multirow[t]{8}{*}{ 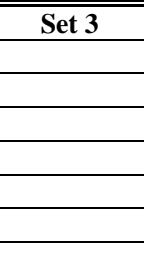 } & "SRM 29253.1 & 1.00 & 60 & 0.05969 & 60 & 0.06062 & 0.98 \\
\hline & SRM 29253.2 & 1.00 & 60 & 0.05028 & 60 & 0.05750 & 0.87 \\
\hline & SRM 29253.3 & 1.00 & 60 & 0.05926 & 60 & 0.06053 & 0.98 \\
\hline & SRM 29253.4 & 1.00 & 60 & 0.05730 & 60 & 0.06050 & 0.95 \\
\hline & SRM 29253.5 & 1.00 & 60 & 0.05451 & 60 & 0.06061 & 0.90 \\
\hline & SRM 29253.6 & 1.00 & 60 & 0.05950 & 60 & 0.06078 & 0.98 \\
\hline & SRM 927e 1.1 & 1.00 & 60 & 0.05004 & 60 & 0.05945 & 0.84 \\
\hline & SRM 927e 1.2 & 1.00 & 60 & 0.05912 & 60 & 0.06066 & 0.97 \\
\hline
\end{tabular}

Table D10: Liquid chromatography gradient for amino acid analysis

\begin{tabular}{|c|c|c|c|c|}
\hline $\begin{array}{c}\text { Time } \\
\text { (min) }\end{array}$ & $\begin{array}{c}\text { Solvent B } \\
\text { \% }\end{array}$ & $\begin{array}{c}\text { Solvent A } \\
\text { \% }\end{array}$ & $\begin{array}{c}\text { Flowrate } \\
(\mathbf{m L / m i n})\end{array}$ & $\begin{array}{c}\text { Pressure } \\
\text { Limit }\end{array}$ \\
\hline \hline $\mathbf{0}$ & 0 & 100 & 0.10 & 400 \\
\hline $\mathbf{2 0}$ & 50 & 50 & 0.10 & 400 \\
\hline $\mathbf{2 2}$ & 100 & 0 & 0.10 & 400 \\
\hline $\mathbf{2 5}$ & 100 & 0 & 0.10 & 400 \\
\hline $\mathbf{3 0}$ & 0 & 100 & 0.10 & 400 \\
\hline $\mathbf{4 0}$ & 0 & 100 & 0.10 & 400 \\
\hline
\end{tabular}


Table D11: Tandem MS MRM fragmentation transitions for amino acid analysis

\begin{tabular}{|c|c|c|c|c|c|}
\hline Measurand & $\begin{array}{c}\text { Internal } \\
\text { Standard (IS) }\end{array}$ & $\begin{array}{c}\text { Precursor Ion mass- } \\
\text { to-charge ratio }\end{array}$ & $\begin{array}{c}\text { Product Ion mass- } \\
\text { to-charge ratio }\end{array}$ & $\begin{array}{c}\text { Collision } \\
\text { Energy (V) }\end{array}$ & $\begin{array}{c}\text { Fragmentor } \\
\text { Voltage (V) }\end{array}$ \\
\hline \hline Alanine & & 90.1 & 44.1 & 8 & 55 \\
\hline Alanine* & yes & 92.1 & 45.1 & 8 & 55 \\
\hline Phenylalanine & & 166.1 & 120.1 & 6 & 85 \\
\hline Phenylalanine* & yes & 176.1 & 129.1 & 6 & 85 \\
\hline Arginine & & 175.1 & 70.1 & 21 & 80 \\
\hline Arginine* & yes & 179.1 & 71.1 & 21 & 80 \\
\hline
\end{tabular}

Table D12: Molecular Mass Calculation of Mature, Native HSA (see Figure D2 for HSA amino acid sequence)

\begin{tabular}{|c|c|c|c|c|}
\hline Chemical Element & $\begin{array}{c}\text { Total Number of Chemical } \\
\text { Elements in HSA }\end{array}$ & $\begin{array}{c}\text { Mass of Chemical } \\
\text { Element }{ }^{\mathbf{a}}(\mathbf{g} / \mathbf{m o l})\end{array}$ & $\begin{array}{c}\text { Total Mass of Element in } \\
\text { HSA (g/mol) }\end{array}$ & $\begin{array}{c}\text { Total Mass of Mature, } \\
\text { Native HSA (g/mol) }\end{array}$ \\
\hline \hline Carbon $\left({ }^{\mathbf{1}} \mathbf{C}\right)$ & 2936 & 12.0000 & 35232.00 & $\mathbf{6 6 , 3 9 4 . 5 6}$ \\
\hline Nitrogen $\left({ }^{\mathbf{1 4}} \mathbf{N}\right)$ & 786 & 14.0031 & 11006.44 & \\
\hline Oxygen $\left({ }^{\mathbf{1 6}} \mathbf{O}\right)$ & 889 & 15.9949 & 14219.47 & \\
\hline Sulfur $\left({ }^{\mathbf{3 2}} \mathbf{S}\right)$ & 41 & 31.9721 & 1310.86 & \\
\hline Hydrogen $\left({ }^{\mathbf{1}} \mathbf{H}\right)$ & 4590 & 1.0078 & 4625.80 & \\
\hline
\end{tabular}

Table D13-A: SRM 2925 molar concentration (nmol/g) for Sample Set 1 (three amino acids: Ala, Phe, Arg)

\begin{tabular}{|c|c|c|c|c|c|}
\hline $\begin{array}{c}\text { Sample } \\
\text { Set }\end{array}$ & Sample & Replicate & $\begin{array}{c}\text { Alanine } \\
\text { (Ala) } \\
\text { (nmol/g) }\end{array}$ & $\begin{array}{c}\text { Phenylalanine } \\
\text { (Phe) } \\
\text { (nmol/g) }\end{array}$ & $\begin{array}{c}\text { Arginine } \\
\text { (Arg) } \\
\text { (nmol/g) }\end{array}$ \\
\hline \multirow[t]{24}{*}{ Set 1} & SRM 29251.1 & 1 & 14.29 & 14.15 & 14.46 \\
\hline & & 2 & 14.45 & 14.17 & 14.29 \\
\hline & & 3 & 14.41 & 14.19 & 14.33 \\
\hline & & 4 & 14.30 & 14.28 & 14.38 \\
\hline & SRM 29251.2 & 1 & 14.32 & 14.34 & 13.95 \\
\hline & & 2 & 14.36 & 14.26 & 13.84 \\
\hline & & 3 & 14.40 & 14.28 & 13.90 \\
\hline & & 4 & 14.38 & 14.30 & 13.94 \\
\hline & SRM 29251.3 & 1 & 14.21 & 14.14 & 13.78 \\
\hline & & 2 & 14.15 & 14.11 & 13.77 \\
\hline & & 3 & 14.13 & 14.11 & 13.94 \\
\hline & & 4 & 14.15 & 14.14 & 13.64 \\
\hline & SRM 29251.4 & 1 & 14.10 & 14.22 & 13.84 \\
\hline & & 2 & 14.28 & 14.18 & 13.89 \\
\hline & & 3 & 14.12 & 13.86 & 13.98 \\
\hline & & 4 & 14.22 & 14.17 & 13.87 \\
\hline & SRM 29251.5 & 1 & 14.22 & 14.08 & 14.01 \\
\hline & & 2 & 14.29 & 14.09 & 13.97 \\
\hline & & 3 & 14.22 & 14.12 & 13.96 \\
\hline & & 4 & 14.26 & 14.25 & 13.93 \\
\hline & SRM 29251.6 & 1 & 14.24 & 14.18 & 13.63 \\
\hline & & 2 & 14.34 & 14.00 & 13.66 \\
\hline & & 3 & 14.13 & 14.02 & 13.72 \\
\hline & & 4 & 14.37 & 14.14 & 13.57 \\
\hline
\end{tabular}


Table D13-B: SRM 2925 molar concentration (nmol/g) for Sample Set 2 (three amino acids: Ala, Phe, Arg)

\begin{tabular}{|c|c|c|c|c|c|}
\hline $\begin{array}{c}\text { Sample } \\
\text { Set }\end{array}$ & Sample & Replicate & $\begin{array}{c}\text { Alanine } \\
\text { (Ala) } \\
\text { (nmol/g) }\end{array}$ & $\begin{array}{c}\text { Phenylalanine } \\
\text { (Phe) } \\
\text { (nmol/g) }\end{array}$ & $\begin{array}{c}\text { Arginine } \\
\text { (Arg) } \\
\text { (nmol/g) }\end{array}$ \\
\hline \multirow[t]{24}{*}{ Set 2} & SRM 29251.1 & 1 & 14.50 & 14.57 & 13.99 \\
\hline & & 2 & 14.36 & 14.49 & 14.14 \\
\hline & & 3 & 14.30 & 14.49 & 14.10 \\
\hline & & 4 & 14.47 & 14.59 & 13.94 \\
\hline & SRM 29251.2 & 1 & 14.51 & 14.57 & 14.46 \\
\hline & & 2 & 14.37 & 14.62 & 14.36 \\
\hline & & 3 & 14.43 & 14.53 & 14.35 \\
\hline & & 4 & 14.54 & 14.54 & 14.40 \\
\hline & SRM 29251.3 & 1 & 14.60 & 14.67 & 14.58 \\
\hline & & 2 & 14.61 & 14.66 & 14.51 \\
\hline & & 3 & 14.76 & 14.76 & 14.38 \\
\hline & & 4 & 14.45 & 14.60 & 14.47 \\
\hline & SRM 29251.4 & 1 & 14.60 & 14.58 & 14.38 \\
\hline & & 2 & 14.71 & 14.63 & 14.41 \\
\hline & & 3 & 14.50 & 14.68 & 14.39 \\
\hline & & 4 & 14.74 & 14.62 & 14.41 \\
\hline & SRM 29251.5 & 1 & 14.42 & 14.53 & 14.52 \\
\hline & & 2 & 14.29 & 14.48 & 14.10 \\
\hline & & 3 & 14.44 & 14.62 & 14.32 \\
\hline & & 4 & 14.43 & 14.55 & 14.33 \\
\hline & SRM 29251.6 & 1 & 14.70 & 14.51 & 14.45 \\
\hline & & 2 & 14.53 & 14.47 & 14.50 \\
\hline & & 3 & 14.38 & 14.39 & 14.31 \\
\hline & & 4 & 14.46 & 14.55 & 14.35 \\
\hline
\end{tabular}

Table D13-C: SRM 2925 molar concentration (nmol/g) for Sample Set 3 (three amino acids: Ala, Phe, Arg).

\begin{tabular}{|c|c|c|c|c|c|}
\hline $\begin{array}{c}\text { Sample } \\
\text { Set }\end{array}$ & Sample & Replicate & $\begin{array}{l}\text { Alanine } \\
\text { (Ala) } \\
\text { (nmol/g) }\end{array}$ & $\begin{array}{c}\text { Phenylalanine } \\
\text { (Phe) } \\
\text { (nmol/g) }\end{array}$ & $\begin{array}{c}\text { Arginine } \\
\text { (Arg) } \\
\text { (nmol/g) }\end{array}$ \\
\hline \multirow[t]{24}{*}{ Set 3} & SRM 29251.1 & 1 & 14.47 & 14.26 & 14.30 \\
\hline & & 2 & 14.43 & 14.55 & 14.20 \\
\hline & & 3 & 14.56 & 14.56 & 14.38 \\
\hline & & 4 & 14.49 & 14.48 & 14.41 \\
\hline & SRM 29251.2 & 1 & 14.35 & 14.31 & 14.83 \\
\hline & & 2 & 14.63 & 14.28 & 14.51 \\
\hline & & 3 & 14.47 & 14.30 & 14.47 \\
\hline & & 4 & 14.27 & 14.29 & 14.50 \\
\hline & SRM 29251.3 & 1 & 15.05 & 14.95 & 15.34 \\
\hline & & 2 & 15.11 & 14.94 & 15.22 \\
\hline & & 3 & 14.92 & 14.83 & 15.26 \\
\hline & & 4 & 14.93 & 14.88 & 15.39 \\
\hline & SRM 29251.4 & 1 & 14.36 & 14.63 & 15.20 \\
\hline & & 2 & 14.58 & 14.39 & 14.82 \\
\hline & & 3 & 14.28 & 14.36 & 14.96 \\
\hline & & 4 & 14.29 & 14.34 & 15.04 \\
\hline & SRM 29251.5 & 1 & 14.79 & 14.80 & 15.39 \\
\hline & & 2 & 14.96 & 15.01 & 14.92 \\
\hline & & 3 & 14.79 & 14.81 & 15.10 \\
\hline & & 4 & 14.65 & 14.64 & 15.12 \\
\hline & SRM 29251.6 & 1 & 14.43 & 14.30 & 15.69 \\
\hline & & 2 & 14.55 & 14.32 & 14.98 \\
\hline & & 3 & 14.32 & 14.30 & 15.10 \\
\hline & & 4 & 14.42 & 14.41 & 14.96 \\
\hline
\end{tabular}


Table D14-A: SRM 927e (QC) concentration for each Sample Set 1 (three amino acids: Ala, Phe, Arg).

\begin{tabular}{|c|c|c|c|c|c|}
\hline $\begin{array}{c}\text { Sample } \\
\text { Set }\end{array}$ & Sample & Replicate & $\begin{array}{c}\text { Alanine } \\
(\mathbf{A l a}) \mathbf{( g / L )}\end{array}$ & $\begin{array}{c}\text { Phenylalanine } \\
(\mathbf{P h e}) \mathbf{( g / L )}\end{array}$ & $\begin{array}{c}\text { Arginine } \\
\text { (Arg) (g/L) }\end{array}$ \\
\hline \hline Set 1 & SRM 927e 1.1 & 1 & 68.73 & 69.85 & 67.01 \\
\hline & & 2 & 69.13 & 70.20 & 67.49 \\
\hline & & 3 & 68.98 & 69.75 & 67.77 \\
\hline & & 4 & 69.01 & 69.64 & 66.90 \\
\hline & SRM 927e 1.2 & 1 & 68.84 & 70.31 & 67.22 \\
\hline & & 2 & 69.26 & 70.48 & 67.77 \\
\hline & & 3 & 68.28 & 70.37 & 67.61 \\
\hline & & 4 & 68.52 & 69.90 & 67.57 \\
\hline & & 1 & 67.41 & 68.24 & 64.85 \\
\hline & & 2 & 67.13 & 68.38 & 66.07 \\
\hline & & 3 & 67.28 & 68.00 & 65.67 \\
\hline & & 4 & 67.64 & 67.92 & 65.86 \\
\hline & & 1 & 67.22 & 68.38 & 66.21 \\
\hline & SRM 927e 2.1 & 2 & 67.74 & 68.88 & 66.15 \\
\hline & & 3 & 67.47 & 68.16 & 66.46 \\
\hline & & 4 & 67.39 & 67.55 & 66.01 \\
\hline
\end{tabular}

Table D14-B: SRM 927e (QC) concentration for each Sample Set 2 (three amino acids: Ala, Phe, Arg).

\begin{tabular}{|c|c|c|c|c|c|}
\hline Sample Set & Sample & Replicate & $\begin{array}{c}\text { Alanine } \\
(\mathbf{A l a}) \mathbf{( g / L )}\end{array}$ & $\begin{array}{c}\text { Phenylalanine (Phe) } \\
\mathbf{( g / \mathbf { L } )}\end{array}$ & $\begin{array}{c}\text { Arginine } \\
\mathbf{( A r g )} \mathbf{( g / L )}\end{array}$ \\
\hline \hline Set 2 & SRM 927e 1.1 & 1 & 70.34 & 71.50 & 68.47 \\
\hline & & 2 & 70.48 & 71.24 & 69.20 \\
\hline & & 3 & 69.38 & 70.72 & 68.80 \\
\hline & & 4 & 69.77 & 70.88 & 68.84 \\
\hline & SRM 927e 1.2 & 1 & 69.85 & 71.18 & 69.12 \\
\hline & & 2 & 71.47 & 70.89 & 69.03 \\
\hline & & 3 & 70.86 & 71.32 & 68.73 \\
\hline & & 4 & 69.21 & 70.26 & 68.84 \\
\hline & SRM 927e 2.1 & 1 & 68.64 & 69.54 & 67.47 \\
\hline & & 2 & 68.50 & 69.22 & 67.22 \\
\hline & & 3 & 69.00 & 69.26 & 67.17 \\
\hline & & 4 & 68.06 & 68.53 & 66.74 \\
\hline & SRM 927e 2.2 & 1 & 68.22 & 68.78 & 66.96 \\
\hline & & 2 & 68.96 & 68.99 & 67.24 \\
\hline & & 3 & 69.37 & 69.59 & 66.50 \\
\hline & & 4 & 69.27 & 69.15 & 66.78 \\
\hline
\end{tabular}

Table D14-C: SRM 927e (QC) concentration for each Sample Set 3 (three amino acids: Ala, Phe, Arg).

\begin{tabular}{|c|c|c|c|c|c|}
\hline Sample Set & Sample & Replicate & $\begin{array}{c}\text { Alanine (Ala) } \\
\mathbf{( g / L )}\end{array}$ & $\begin{array}{c}\text { Phenylalanine } \\
\text { (Phe) (g/L) }\end{array}$ & $\begin{array}{c}\text { Arginine } \\
\text { (Arg) (g/L) }\end{array}$ \\
\hline \hline Set 3 & SRM 927e 1.1 & 1 & 69.36 & 69.87 & 69.17 \\
\hline & & 2 & 70.68 & 70.26 & 69.29 \\
\hline & & 3 & 71.26 & 71.12 & 69.00 \\
\hline & & 4 & 69.73 & 69.09 & 69.46 \\
\hline & SRM 927e 1.2 & 1 & 70.27 & 69.33 & 69.97 \\
\hline & & 2 & 70.89 & 69.85 & 69.17 \\
\hline & & 3 & 71.15 & 70.94 & 69.91 \\
\hline & & 4 & 68.97 & 70.55 & 69.87 \\
\hline & SRM 927e 2.1 & 1 & 68.25 & 67.90 & 67.27 \\
\hline & & 2 & 68.39 & 68.15 & 67.64 \\
\hline & & 3 & 68.13 & 68.05 & 67.67 \\
\hline & & 4 & 66.29 & 67.91 & 67.18 \\
\hline & & 1 & 67.40 & 67.33 & 66.73 \\
\hline & SRM 927e 2.2 & 2 & 68.63 & 68.46 & 67.61 \\
\hline & & 3 & 67.77 & 68.56 & 66.66 \\
\hline & & 4 & 65.56 & 67.51 & 66.58 \\
\hline
\end{tabular}


Table D15: Molar concentration of HSA in SRM 2925 for three (3) amino acids (Ala, Phe, Arg).

\begin{tabular}{|r|c|c|c|c|}
\hline \multicolumn{1}{|c|}{ Recombinant HSA in SRM 2925 } & Set 1 & Set 2 & Set 3 & Average (Set 1-3) \\
\hline \hline Average Recombinant HSA concentration (nmol/g) & 14.1 & 14.5 & 14.7 & 14.4 \\
\hline Standard Deviation (nmol/g) & 0.2 & 0.2 & 0.4 & 0.3 \\
\hline $\mathbf{\% C V}$ & 1.5 & 1.1 & 2.4 & 2.4 \\
\hline
\end{tabular}

Table D16: Mass concentration values for SRM 927e for three (3) amino acids (Ala, Phe, Arg).

\begin{tabular}{|r|c|c|c|c|}
\hline SRM 927e (QC Material) & Set 1 & Set 2 & Set 3 & Average (Set 1-3) \\
\hline \hline Average BSA Concentration (g/L) & 68.77 & 70.01 & 69.96 & $\mathbf{6 8 . 6 3}$ \\
\hline Standard Deviation (g/L) & 1.15 & 1.02 & 0.75 & $\mathbf{1 . 4 5}$ \\
\hline \%CV & 1.7 & 1.5 & 1.1 & $\mathbf{2 . 1}$ \\
\hline
\end{tabular}

\footnotetext{
1 MKWVTFISLLFLFSSAYSRGVFRRDAHKSEVAHRFKDLGEENFKALVLIAFAQ

54 YLQQCPFEDHVKLVNEVTEFAKTCVADESAENGDKSLHTLFGDKLGTVATLRE

107 TYGEMADCGAKQE PERNECFLQHKDDNPNLPRLVYRPEVDVMCTA,FHDNEETFL

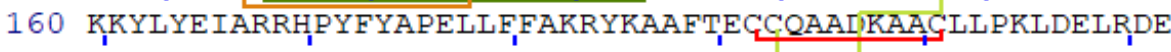

213 GKASSAKQRLKGASLQKF,GERAFKAWAVARLSQRFPKAEFAEVSKLVT,DLTKV

266 HTECCHGD LLECADDRADLAKY ICENNQDSISSKLKY ECCEKPLLEK,SHGIAEVE

319 NDEMPADLPSLAADFVESKDVGGKNYAEAKDVF,LGMFLYEYARRHPDYSVVLLI

372 RLAKTYETT,LEKCCAAA P,HECYAKVFDEFKPLVEE PQN I IKQNCELFEQLGE

425 YKFQNALLLVRYTKKVP,QVSTPTLVEV,SRNLGKVGSKCCKHPEAKRMPGAEDYL

478 SVVLNQLCVLHEKT PVSDRVTKCGTESLVNRRPCFSALEVDETYYVPKEFNAET,

531 FTFHADICTLSEKERQIKKQTALVELVKHKPKATKEQLKA,VMDDFAAFVE,KCC

584 KADDKET, CFAEEGKKLVAASQAALGL
}

Figure D1. Amino acid sequence of HSA protein in SRM 2925 according to the UniProt database (P02768), including the 17 disulfide bonds between cysteine residues: 77-86, 99-115, 114-125, 148-193, 192-201, 224-270, 269-277, 289-303, 302-313, 340-385, 384-393, 416-462, 461-472, 485-501, 500-511, 538-583, and 582-591. The sequence of the mature protein (amino acid residues 25-609) is represented in black and the signal peptide and propeptide (amino acid residues 1-24) are both represented in yellow.

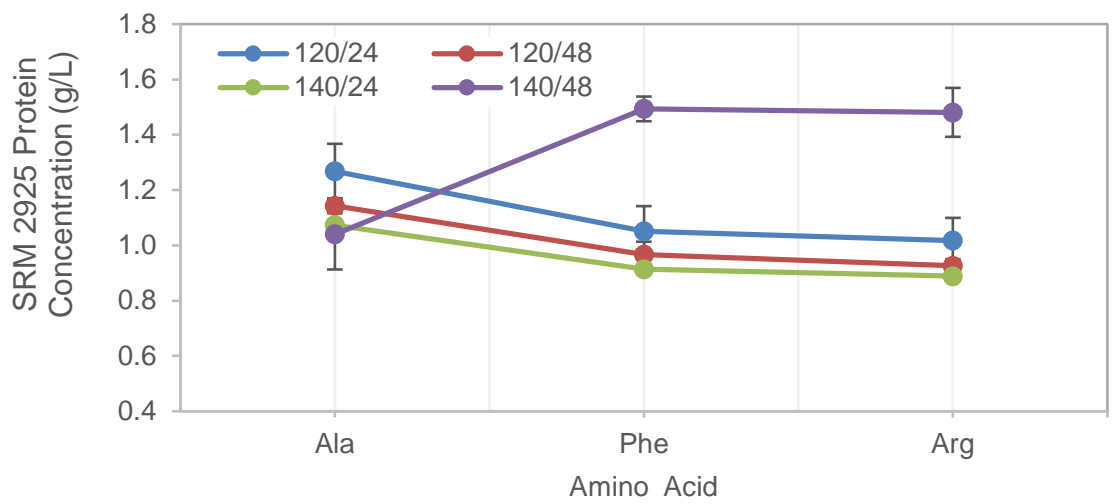

Figure D2. Optimization of hydrolysis conditions (reaction temperature and time) for SRM $2925(\mathrm{n}=3)$ 


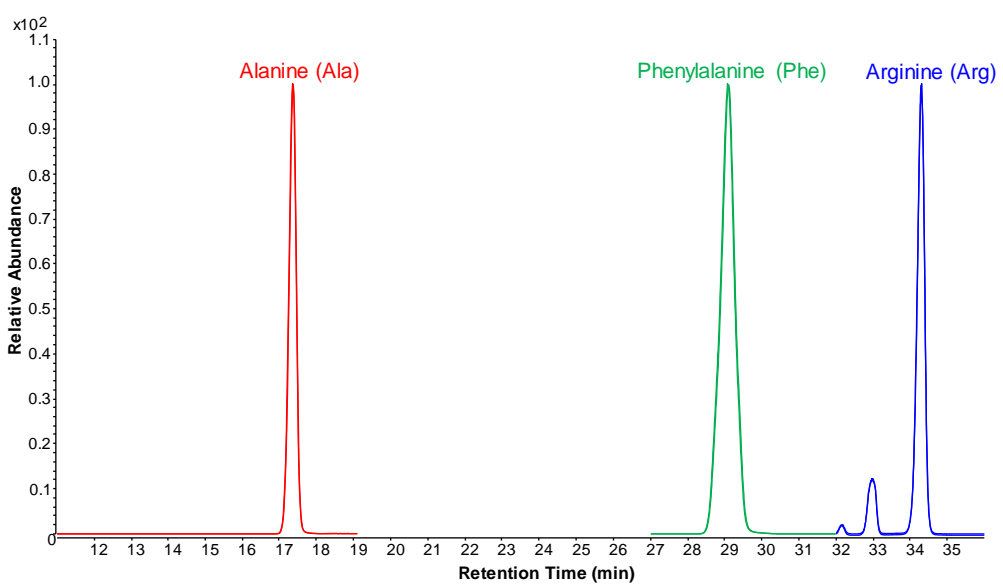

Figure D3-A. Total Ion Chromatogram (TIC) of amino acid analysis of calibration blend (Calibrant 5.1).

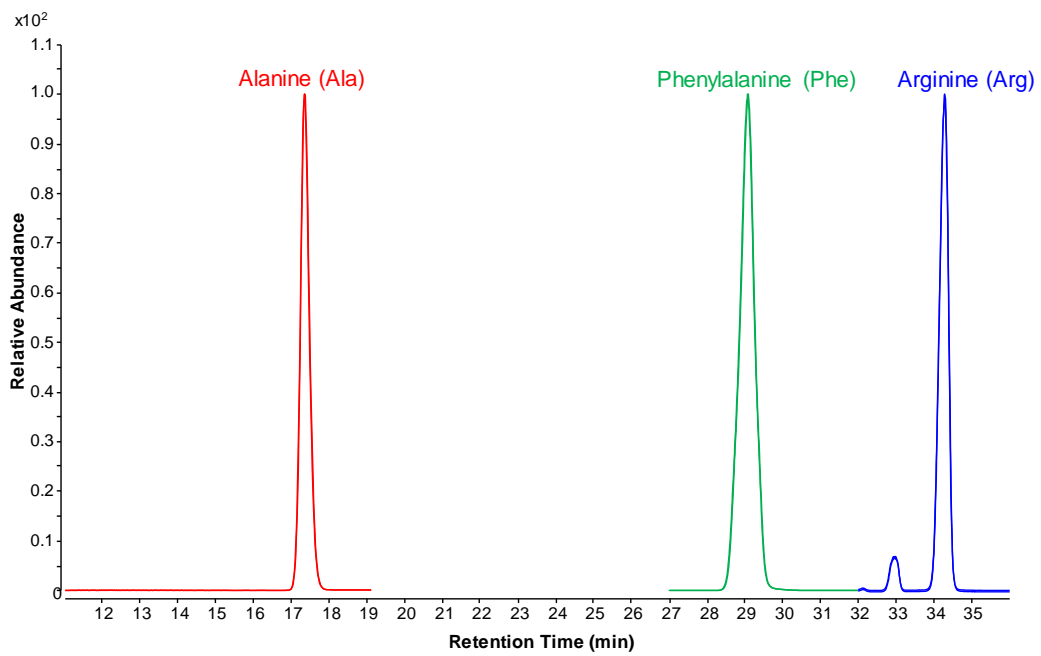

Figure D3-B. Total Ion Chromatogram (TIC) of amino acid analysis of SRM 2925 (SRM 2925 4.1, Set 1). 


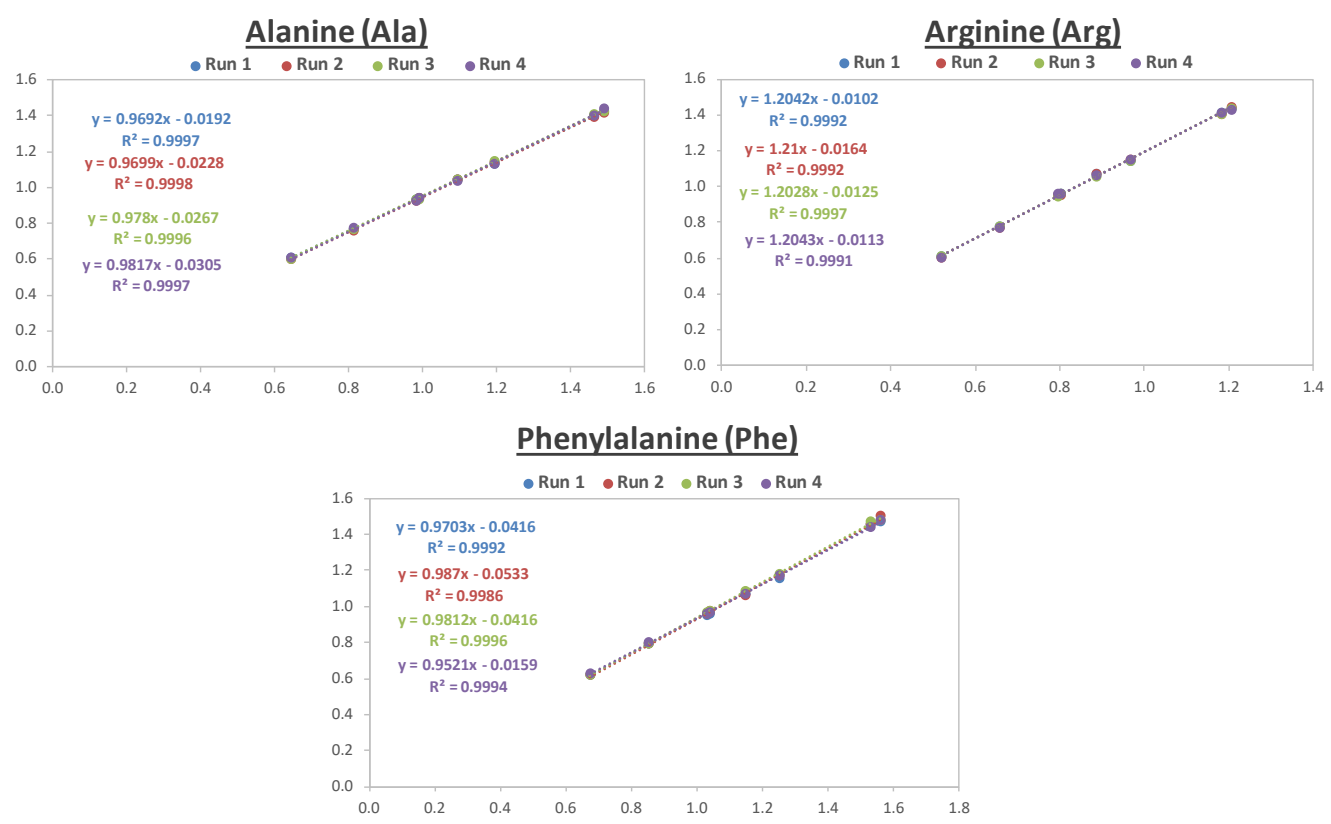

Figure D4. Sample set 1 calibration curves for Alanine (Ala), Phenylalanine (Phe), and Arginine (Arg). (Run 1-4)

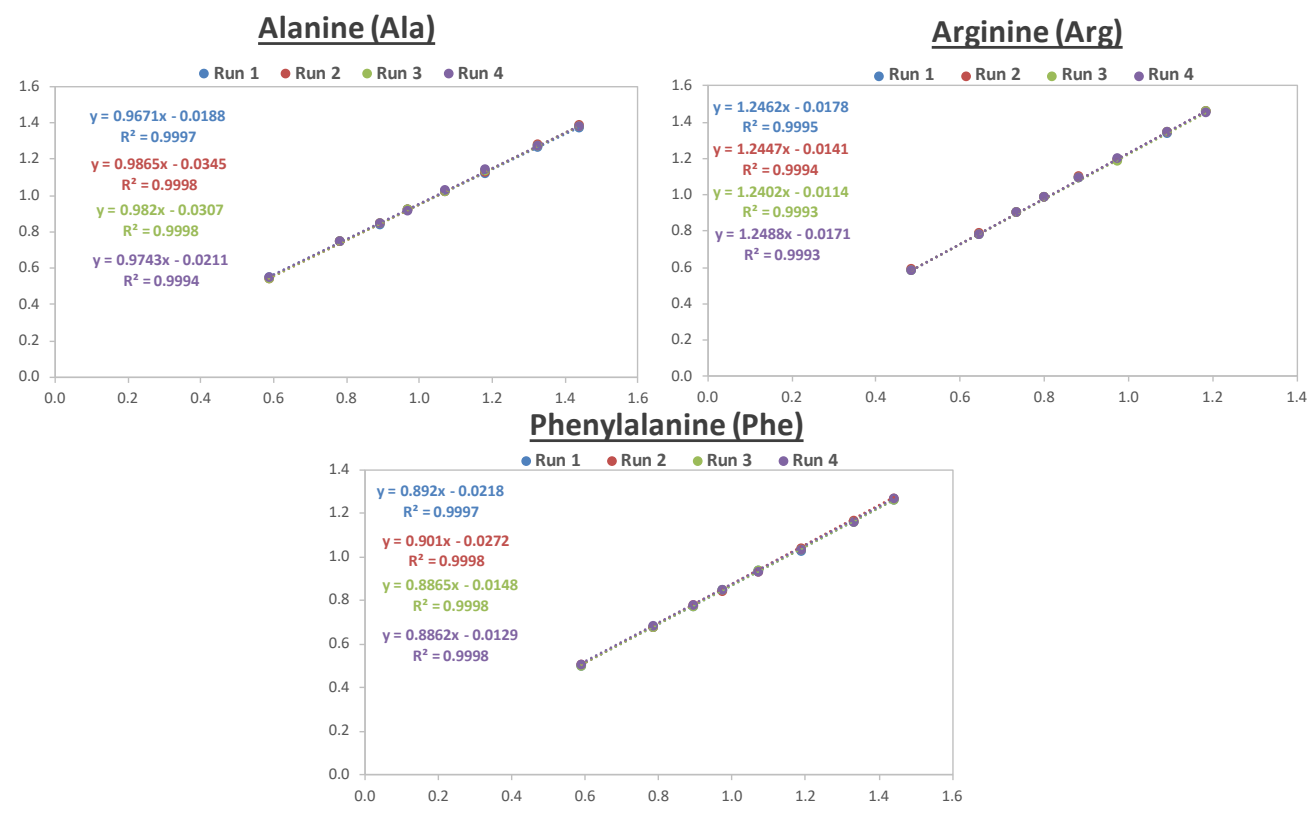

Figure D5. Sample set 2 calibration curves for Alanine (Ala), Phenylalanine (Phe), and Arginine (Arg). (Run 1-4) 


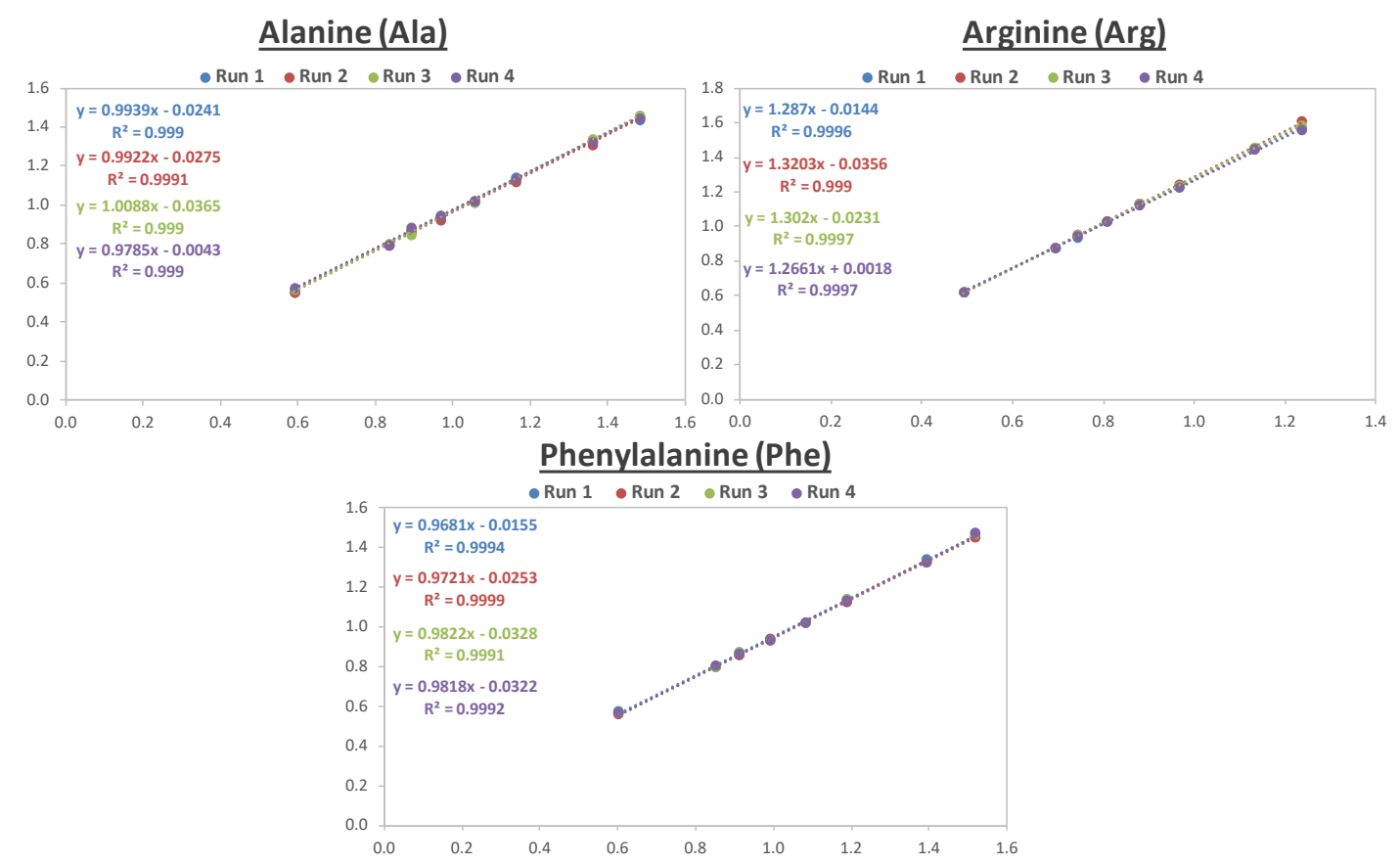

Figure D6. Sample set 3 calibration curves for Alanine (Ala), Phenylalanine (Phe), and Arginine (Arg). (Run 1-4) 


\section{APPENDIX E: Density Measurements of SRM 2925}

\section{INTRODUCTION}

This report describes the density measurements for SRM 2925 to facilitate conversion of mass fraction unit to mass concentration unit, which is commonly used by the clinical measurement community.

\section{EXPERIMENTAL}

Materials. NIST SRM 2925 Recombinant Human Serum Albumin (Primary Reference Calibrator for Urine Albumin) (Frozen)

Samples. A total of six (6) SRM 2925 samples were selected from the lot via a stratified random sampling scheme for analysis.

Density Measurements. The density measurements were conducted on the Anton Paar DMA 5000M Density Meter (serial \#81101696) at $20.0^{\circ} \mathrm{C}$. The density meter has a resolution of $0.000001 \mathrm{~g} / \mathrm{mL}$ for density and of $0.001{ }^{\circ} \mathrm{C}$ for temperature. These resolutions are adequate for the density determinations required for SRM 2925.

A total of six (6) SRM 2925 samples were randomly selected and four technical replicates were analyzed to evaluate the technical variation of the density meter. A $1 \mathrm{~mL}$ test portion of each sample was carefully injected onto the density meter in a manner that minimized the production of air bubbles. Initial density measurements of water $\left(20.0^{\circ} \mathrm{C}\right)$ were performed to determine if the results from the meter were fit-forpurpose prior to analysis of the SRM 2925 samples.

\section{RESULTS AND DISCUSSION}

The density measurements for SRM 2925 are shown in Table E1. The density for the water control is shown compared to the literature value at $20^{\circ} \mathrm{C}$ [1] in Table E2. The within- and between-sample density measurements for SRM 2925 are reproducible with high precision across the six samples (Table E1). The overall density for SRM 2925 at $20.0^{\circ} \mathrm{C}$ was determined to be $1.00016 \mathrm{~g} / \mathrm{mL} \pm 0.00001 \mathrm{~g} / \mathrm{mL}$.

\section{REFERENCES}

E1. Perry, R. H., Green, D. W. and Maloney J. O. Perry's Chemical Engineers' Handbook, Seventh Edition, McGraw-Hill, 1997, Section 2. 
Table E1. Density measurements for SRM 2925 (20.0 $\left.{ }^{\circ} \mathrm{C}\right)$.

\begin{tabular}{|r|c|c|c|c|c|c|}
\hline & \multicolumn{7}{|c|}{ 2925 Density Measurement (g/mL) } \\
\hline Technical Replicate & $\mathbf{2 9 2 5 - 1}$ & $\mathbf{2 9 2 5}-\mathbf{2}$ & $\mathbf{2 9 2 5}-\mathbf{3}$ & $\mathbf{2 9 2 5}-\mathbf{4}$ & $\mathbf{2 9 2 5}-\mathbf{5}$ & $\mathbf{2 9 2 5}-\mathbf{6}$ \\
\hline $\mathbf{1}$ & 1.000159 & 1.000161 & 1.000156 & 1.000160 & 1.000149 & 1.000152 \\
\hline $\mathbf{2}$ & 1.000161 & 1.000161 & 1.000157 & 1.000160 & 1.000149 & 1.000152 \\
\hline $\mathbf{3}$ & 1.000162 & 1.000161 & 1.000157 & 1.000160 & 1.000150 & 1.000152 \\
\hline $\mathbf{4}$ & 1.000162 & 1.000160 & 1.000158 & 1.000160 & 1.000151 & 1.000152 \\
\hline Average Density (g/mL) & 1.000161 & 1.000161 & 1.000157 & 1.000160 & 1.000150 & 1.000152 \\
\hline $\mathbf{S t d e V}$ & 0.000001 & 0.000001 & 0.000001 & 0.000000 & 0.000001 & 0.000000 \\
\hline $\mathbf{0 C V}$ & 0.00 & 0.00 & 0.00 & 0.00 & 0.00 & 0.00 \\
\hline Average Density (g/mL) & $\mathbf{1 . 0 0 0 1 5 7}$ & & & & & \\
\hline Stdev & $\mathbf{0 . 0 0 0 0 0 5}$ & & & & & \\
\hline $\mathbf{0 C V}$ & $\mathbf{0 . 0 0}$ & & & & & \\
\hline
\end{tabular}

Table E2. Density measurements for water control $\left(20.0^{\circ} \mathrm{C}\right)$.

\begin{tabular}{|c|c|}
\hline Water Replicate & Density (g/mL) \\
\hline 1 & 0.998217 \\
\hline 2 & 0.998226 \\
\hline 3 & 0.998216 \\
\hline 4 & 0.998220 \\
\hline 5 & 0.998223 \\
\hline 6 & 0.998224 \\
\hline Average Density (g/mL) & 0.998221 \\
\hline Stdev & 0.000004 \\
\hline$\% \mathrm{CV}$ & 0.0004 \\
\hline Literature Value $\left(20.0^{\circ} \mathrm{C}\right)[1]$ & 0.998204 \\
\hline
\end{tabular}

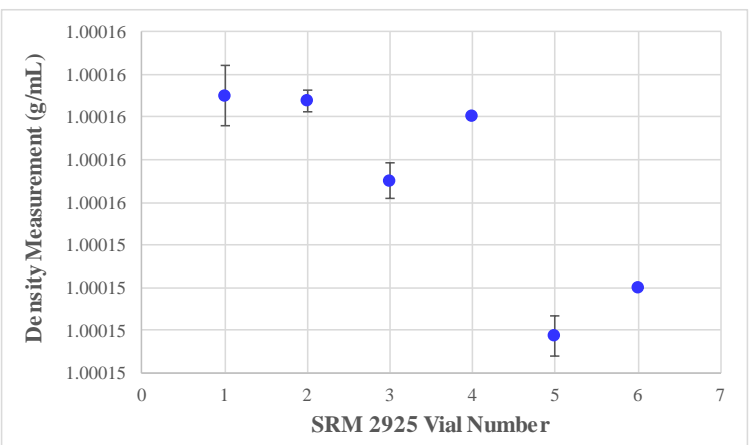

Figure E1. Density measurements for each vial of SRM $2925\left(20.0^{\circ} \mathrm{C}\right)$. 


\section{APPENDIX F: Assessment of Disulfide Bond Profile of SRM 2925}

\section{INTRODUCTION}

Disulfide bonds are post-translational modifications in proteins formed between the sulfur atoms of two cysteine (Cys) residues and play a major role in the structure and function of proteins. Structurally, disulfide bonds ensure proper folding of proteins by stabilizing the high-order conformations of proteins (i.e., tertiary protein structure).

Human Serum Albumin (HSA) contains 17 disulfide bridges, which play a role in the stabilization of the native protein structure of HSA and are also involved in the conformational structure to support the binding function (Figure F1). Therefore, this report describes the disulfide bond profile of recombinant HSA in SRM 2925 to ensure proper folding and stability of the native protein structure.

\section{EXPERIMENTAL}

Materials. NIST SRM 2925 Recombinant Human Serum Albumin (Primary Reference Calibrator for Urine Albumin) (Frozen), Trypsin-Gold MS-grade (Promega), ammonium bicarbonate (Millipore SIGMA), dithiothreitol (DTT) (Pierce), iodoacetamide (IAM) (Pierce), high-purity LC-MS grade water with $0.1 \%$ (v/v) formic acid, and acetonitrile with $0.1 \%(\mathrm{v} / \mathrm{v})$ formic acid (Honeywell Burdick and Jackson).

In-Solution Enzymatic Digestion. The SRM 2925 samples were incubated at $97^{\circ} \mathrm{C}$ for 10 min to denature HSA and cooled to room temperature (RT). For the reduced condition, the samples were reduced with 5 $\mathrm{mmol} / \mathrm{L}$ dithiothreitol at $60^{\circ} \mathrm{C}$ for $30 \mathrm{~min}$ followed by alkylation with $15 \mathrm{mmol} / \mathrm{L}$ iodoacetamide at RT for 30 min in the dark. A 1:30 mass ratio of trypsin-to-protein was used for digestion at $37^{\circ} \mathrm{C}$ overnight $(\sim 18$ h). Following the trypsin digestion, the $\mathrm{pH}$ of the samples was reduced with $50 \mathrm{~mL} / \mathrm{L}$ formic acid in water and incubated for $45 \mathrm{~min}$. at $37^{\circ} \mathrm{C}$ to quench the digestion reaction. The samples were concentrated and resuspended in $100 \mu \mathrm{L}$ of $0.1 \%(\mathrm{v} / \mathrm{v})$ formic acid in water for tandem MS analysis.

Liquid Chromatography-Tandem MS Analysis. LC general conditions: Dionex UltiMate 3000 HPLC System (Thermo Fisher; San Jose, CA) using an Agilent Zorbax 300SB-C18 column (2.1 mm x $150 \mathrm{~mm}$, $3.5 \mu \mathrm{m})$. The column temperature was maintained at $40{ }^{\circ} \mathrm{C}$ and the peptides were loaded onto the column with a flowrate of $200 \mu \mathrm{L} / \mathrm{min}$ in $97 \%$ (v/v) Solvent A (water with $1 \mathrm{~mL} / \mathrm{L}$ formic acid) and $3 \%$ (v/v) Solvent B (acetonitrile with $1 \mathrm{~mL} / \mathrm{L}$ formic acid). The peptides were eluted from the stationary phase with a linear gradient and the total gradient time was $115 \mathrm{~min}$. Solvent B was held constant at $3 \%$ for 5 min and then ramped to $10 \%$ in $3 \mathrm{~min}$, to $20 \%$ in $18 \mathrm{~min}$, to $30 \%$ in $18 \mathrm{~min}$, to $40 \%$ in $18 \mathrm{~min}$, to $50 \%$ in $18 \mathrm{~min}$, and to $70 \%$ in $10 \mathrm{~min}$. At $97 \mathrm{~min}$, Solvent B was increased to $97 \%$ and held constant for $5 \mathrm{~min}$ and ramped down to $3 \%$ in 3 min for a 10-min column re-equilibration. Clean and blank runs preceded each analysis set to prevent carry-over.

General conditions for the LTQ Orbitrap Elite ETD (Thermo Fisher; San Jose, CA) linear ion trap mass spectrometer for CID in positive ion mode: Heated ESI, source heater temperature of $300{ }^{\circ} \mathrm{C}$, sheath gas flow rate of 35 (arbitrary units), auxiliary gas flow rate of 10 (arbitrary units), capillary temperature of 350 ${ }^{\circ} \mathrm{C}$, source voltage of $3.80 \mathrm{kV}$, S-lens RF level of $60 \%$, normalized collision energy of $35.0 \%$, default charge state of 2 , activation q-value of 0.25 , and activation time at $10 \mathrm{~ms}$.

The Top 5 data-dependent acquisition (DDA) experiment was used in this study, where the top five most intense ions from the full MS (MS1) scan were targeted for MS/MS (MS2) analysis. The raw tandem MS data was processed manually and with the Protein Discoverer software (Version 2.3, Thermo Fisher), the 
UniProtKB/Swiss-Prot human serum albumin (P02768) fasta from http://uniprot.org created on April 1, 1990 was the fasta file used in the MS/MS search parameters.

\section{RESULTS AND DISCUSSION}

In this report, the disulfide connectivity pattern of recombinant HSA in SRM 2925 was investigated via a bottom-up MS approach, which includes enzymatic digestion followed by LCMS/MS analysis under reduced and non-reduced conditions. The reduced samples were reduced (DTT) and alkylated (iodoacetamide) prior to trypsin digestion; however, the non-reduced samples, were only alkylated with iodoacetamide (to modify the free Cys residues) prior to trypsin digestion. From an in-silico digest of non-reduced HSA, there are eight (8) theoretical disulfidecontaining peptide complexes present in non-reduced HSA (Figure F2). Following trypsin digestion, both sample types (reduced, non-reduced) were analyzed via LC-MS/MS and the chromatograms are shown in Figure F3 and F4 demonstrate the reproducibility of the trypsin digest via process replicates and the LC-MS/MS analysis via technical replicates. From the peptide profile data, the protein sequence coverage for the reduced condition was $96 \%$ and the nonreduced condition was $76 \%$. Comparison of the peptide profiles of the two conditions (reduced vs. non-reduced) reveal the presence of disulfide linkages (Figure F5). Further assessment of the non-reduced tandem MS spectra confirms the presence of six of the eight expected disulfidecontaining peptide complexes (Complex 1, 2, 3, 4, 6, and 8) in SRM 2925 (Figure F2). The full MS and tandem MS spectra corresponding to the six disulfide-containing complexes are shown in Figures F6 to F11. Detection of the six disulfide-containing peptide complexes in the non-reduced samples supports the presence of the expected disulfide connectivity pattern of recombinant HSA in SRM 2925. The peptide identifications for both conditions (reduced vs. non-reduced) are outlined in Appendix F1 to F3. The overlap in peptide identifications within-vial (technical replicates) and between-vial (process replicates) for both conditions (reduced vs. non-reduced) is consistent with the repeatability of the base peak chromatograms observed for each run displayed in Figure F3 and F4.

Therefore, we can determine from the peptide profile and the disulfide connectivity pattern that the recombinant HSA in SRM 2925 is the mature, native form of the protein and the amino acid sequence is consistent with the theoretical sequence for HSA. These results support the use of SRM 2925 as a primary reference material for HSA measurements. 


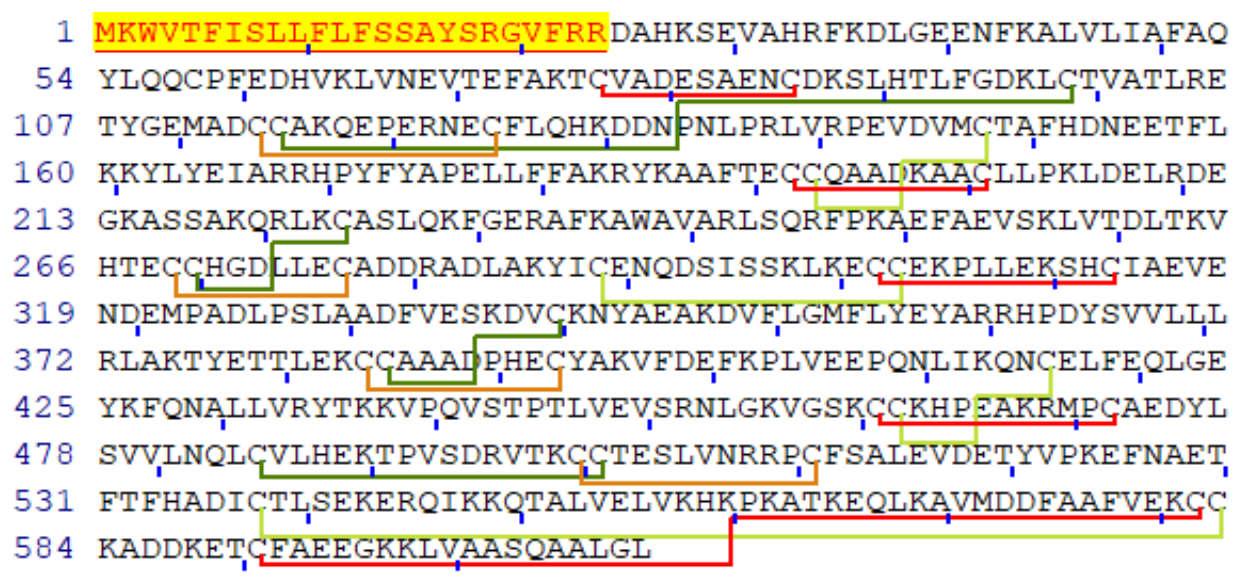

Figure F1. Amino acid sequence of HSA protein in SRM 2925 according to the UniProt database (P02768), including the 17 disulfide bonds between cysteine residues: 77-86, 99-115, 114-125, 148-193, 192-201, 224-270, 269-277, 289-303, 302-313, 340-385, 384-393, 416-462, 461-472, 485-501, 500-511, 538-583, and 582-591. The sequence of the mature protein (amino acid residues 25-609) is represented in black and the signal peptide and propeptide (amino acid residues 1-24) are both represented in yellow.

${ }_{76}$ TCVADESAENCDK

${ }_{98}$ LCTVATLR
${ }_{106}$ ETYGEMADCCAK
${ }_{123}$ NECFLQHK

\author{
${ }_{287}$ YICENQDSISSK \\ ${ }_{301}$ ECCEKPLLEK \\ ${ }_{311}$ SHCI AEVENDEMPADLPSLAADFVESK
}

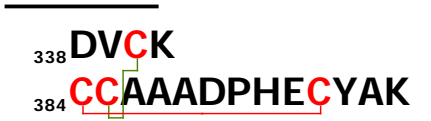

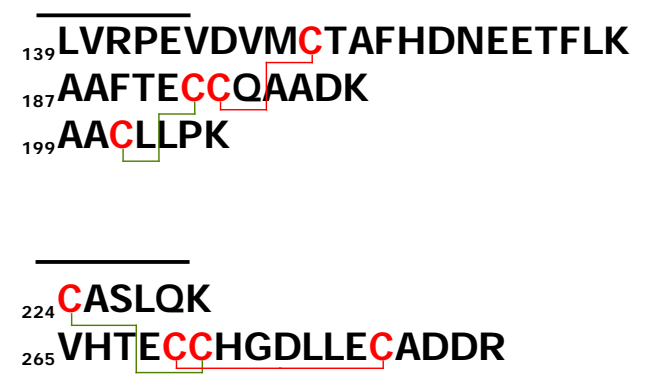

Figure F2. Theoretical disulfide-containing peptide complexes from an in silico tryptic digestion of HSA under non-reducing conditions. 


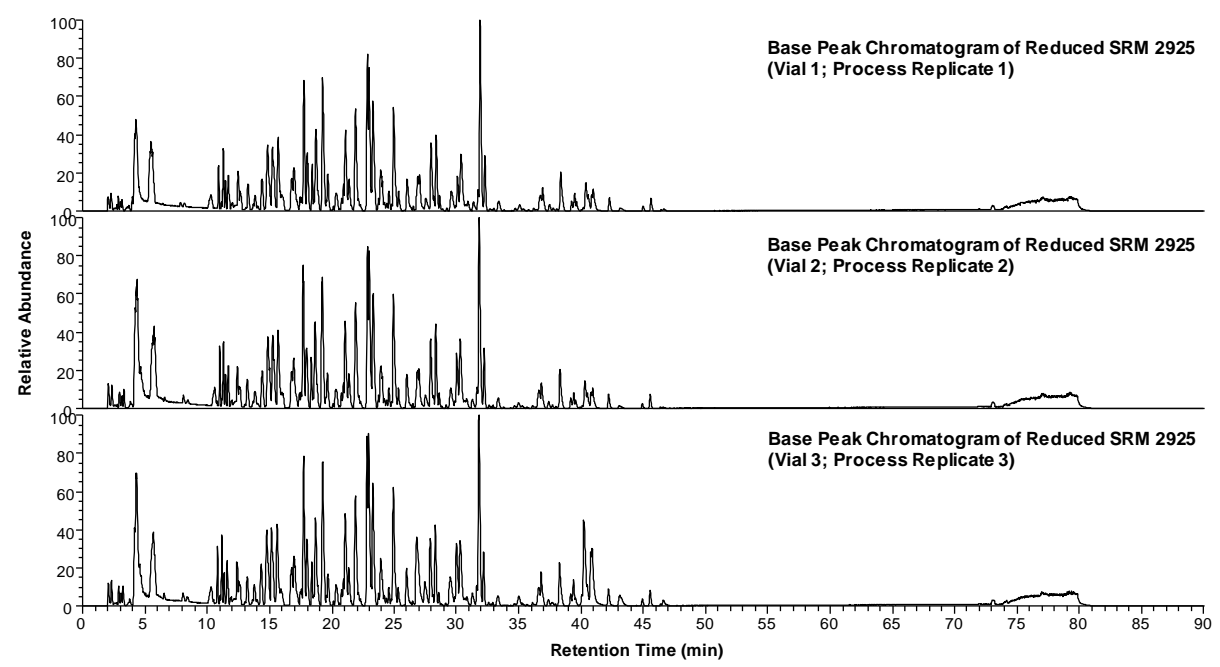

Figure F3-A. Base peak chromatograms of trypsin digest products from recombinant HSA in SRM 2925 under the reduced conditions. Three (3) vials (process replicates) were digested in parallel and the samples were analyzed via tandem MS in four (4) replicates (technical replicates). The chromatograms represented are from the tandem MS analysis of the three (3) process replicates.

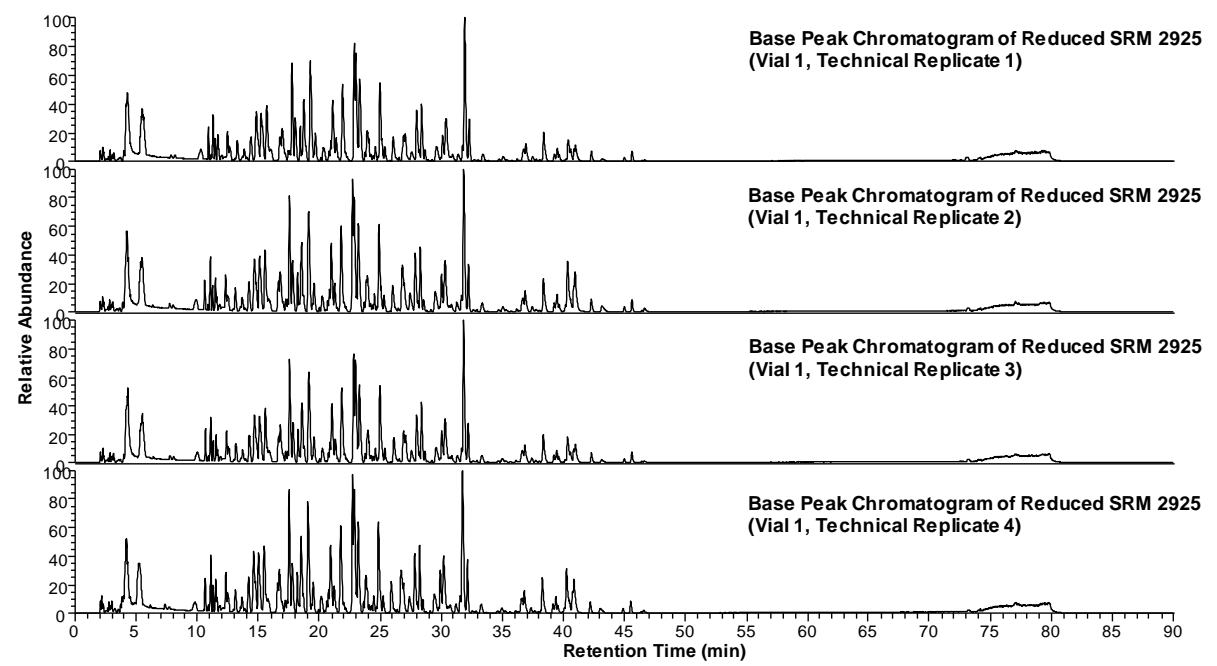

Figure F3-B. Base peak chromatograms of trypsin digest products from recombinant HSA in SRM 2925 under the reduced conditions. Three (3) vials (process replicates) were digested in parallel and the samples were analyzed via tandem MS in four (4) replicates (technical replicates). The chromatograms represented are from the tandem MS analysis of the four (4) technical replicates of Vial 1. 


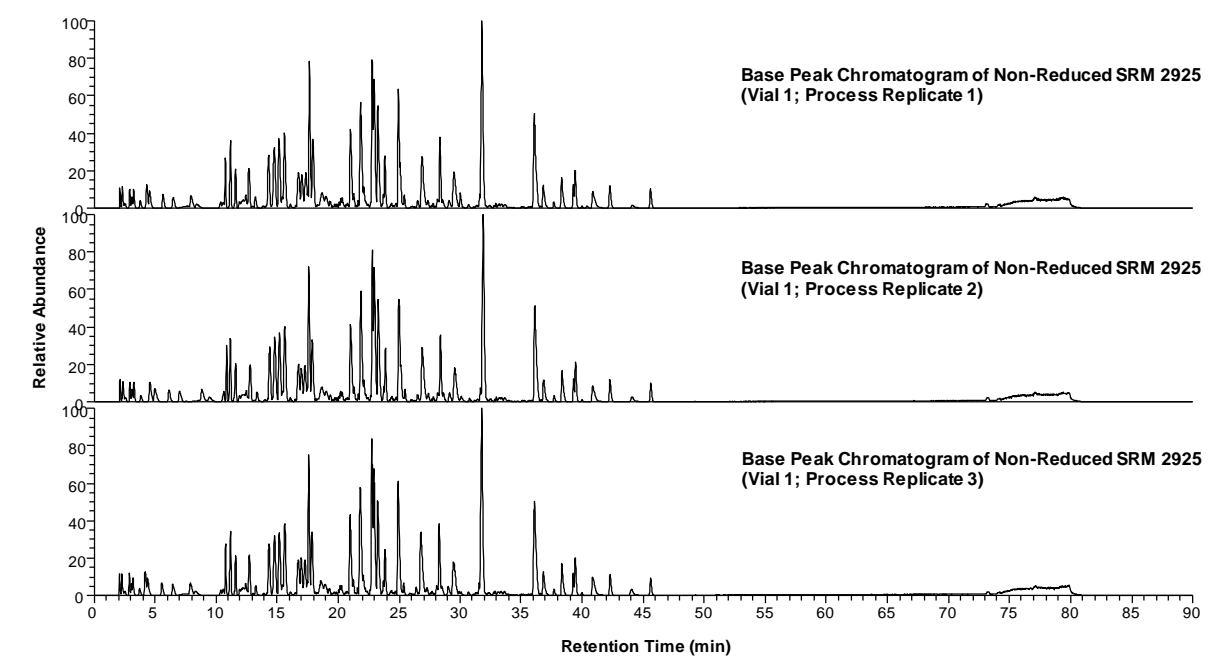

Figure F4-A. Base peak chromatograms of trypsin digest products from recombinant HSA in SRM 2925 under the non-reduced conditions. Three (3) vials (process replicates) were digested in parallel and the samples were analyzed via tandem MS in four (4) replicates (technical replicates). The chromatograms represented are from the tandem MS analysis of the three (3) process replicates.

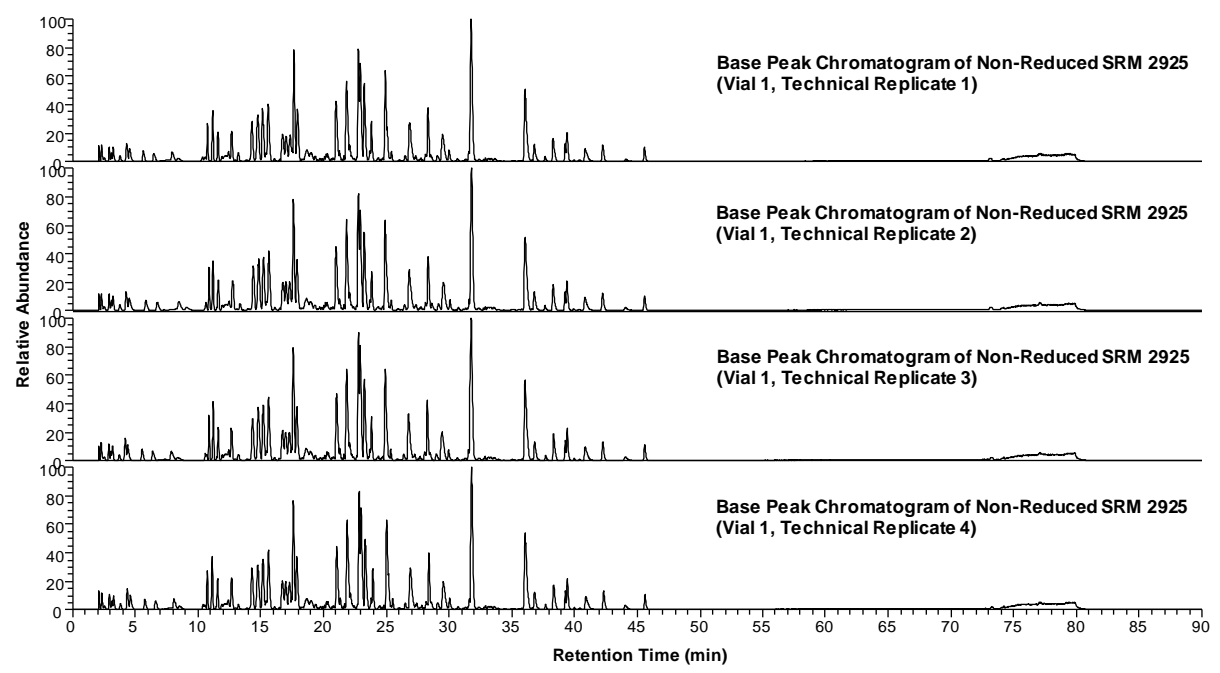

Figure F4-B. Base peak chromatograms of trypsin digest products from recombinant HSA in SRM 2925 under the non-reduced conditions. Three (3) vials (process replicates) were digested in parallel and the samples were analyzed via tandem MS in four (4) replicates (technical replicates). The chromatograms represented are from the tandem MS analysis of the four (4) technical replicates of Vial 1. 


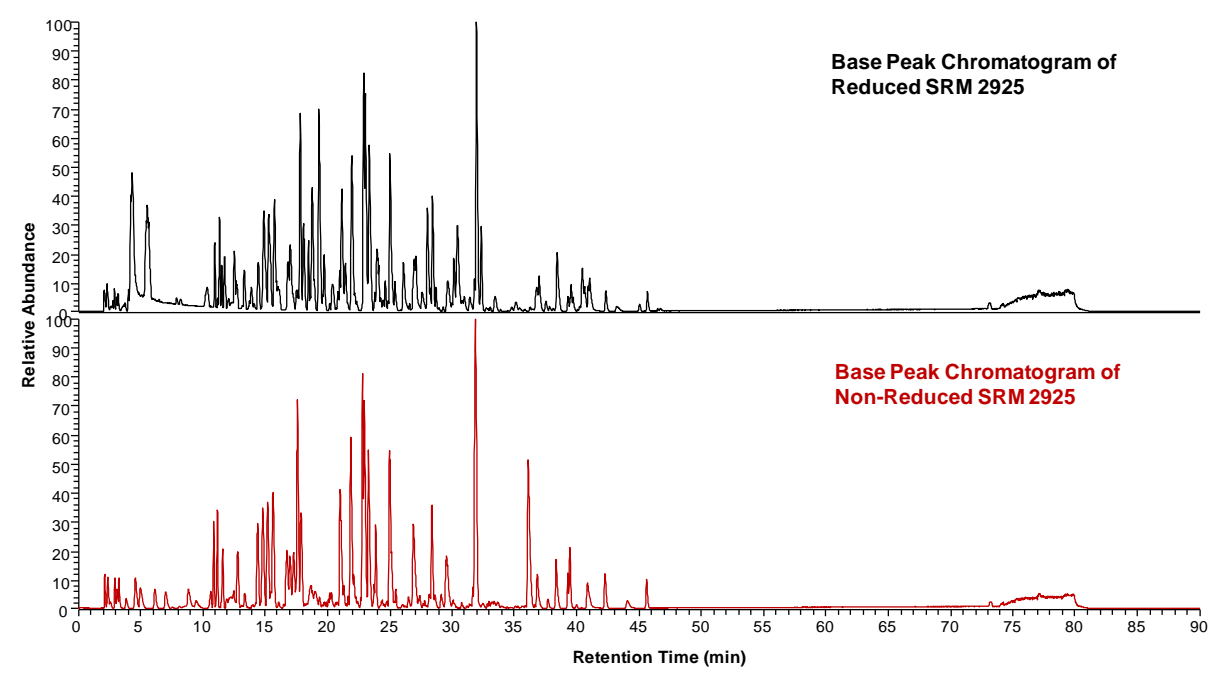

Figure F5. Base peak chromatograms of tandem MS analysis of recombinant HSA trypsin digest products in SRM 2925 under the reduced (black) and non-reduced (red) conditions. 

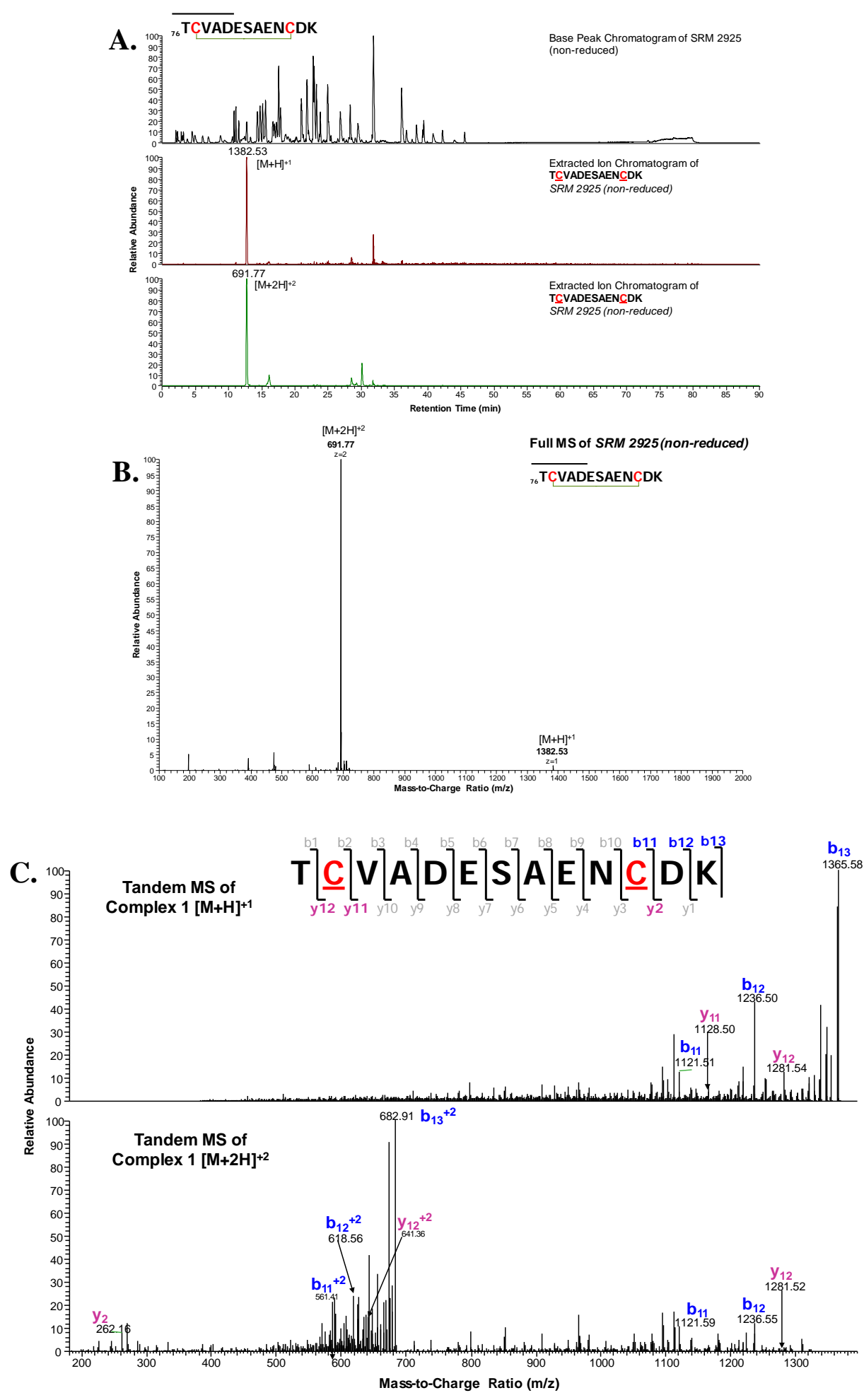

Figure F6. Disulfide profile of HSA Disulfide Complex 1 (see Figure 2) under the non-reduced condition. Base peak chromatogram and extracted-ion chromatograms (XICs) of tandem MS analysis of Disulfide Complex 1 (A), full MS spectrum of Disulfide Complex 1 (B), and tandem MS spectra of Disulfide Complex 1 (single and double charged species) (C). 

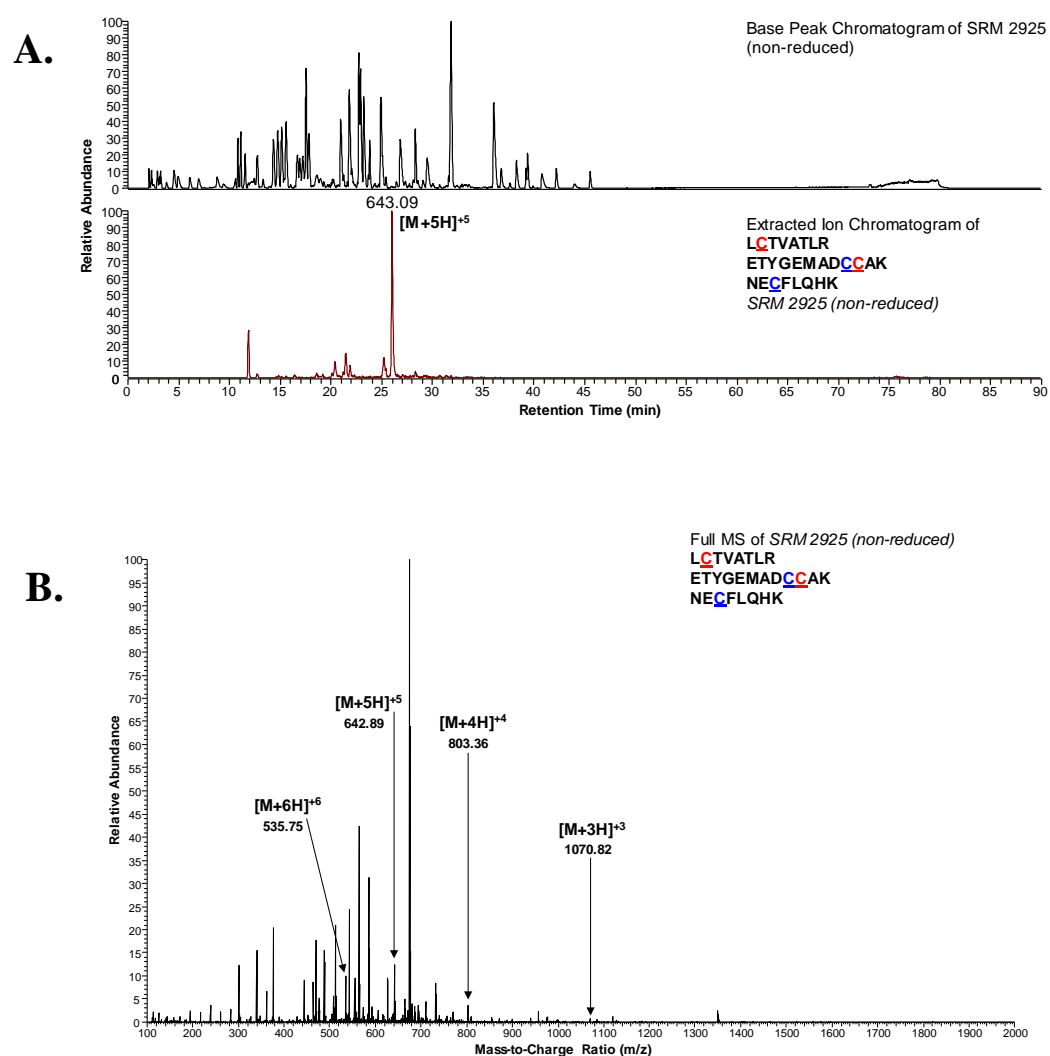

$\mathbf{P 1} \mathbf{L}[\underline{\mathrm{C}}[\mathbf{T}] \mathbf{V}] \mathbf{A}] \mathbf{T}] \mathbf{L}] \mathbf{R}$

P2 EE T] Y Y $] \mathbf{G}] \mathbf{E}[\mathbf{M}] \mathbf{A}] \mathbf{D}] \mathbf{D}] \mathbf{C}] \mathbf{C}] \mathbf{A}] \mathbf{K}$

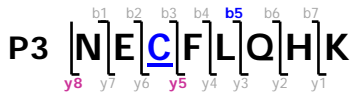

C.

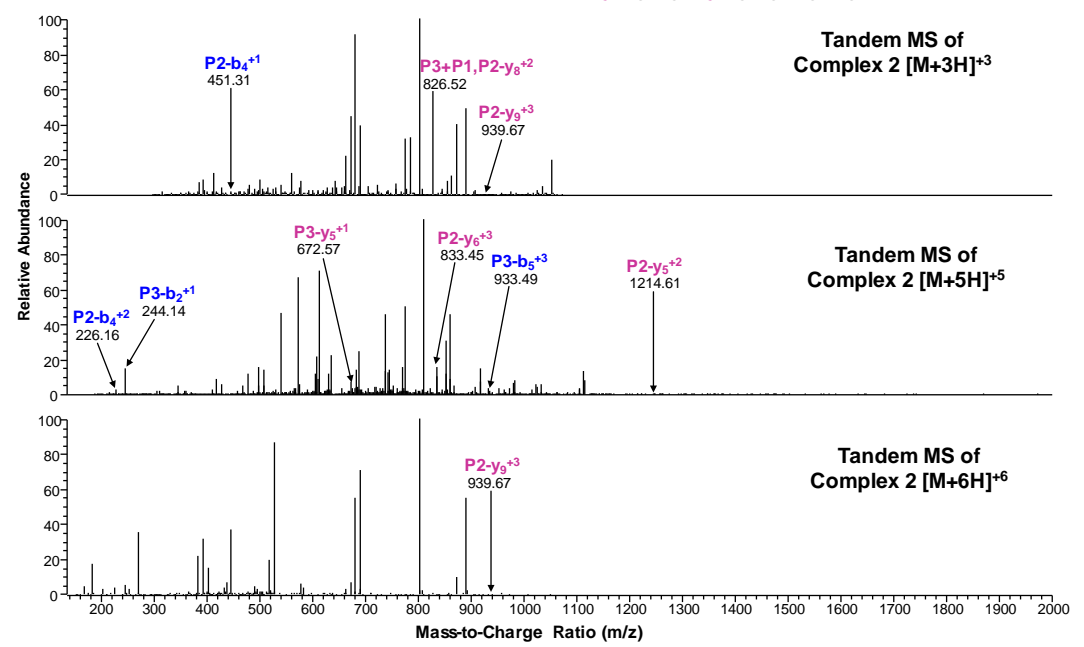

Figure F7. Disulfide profile of HSA Disulfide Complex 2 (see Figure 2) under the non-reduced condition. Base peak chromatogram and extracted-ion chromatograms (XICs) of tandem MS analysis of Disulfide Complex 2 (A), full MS spectrum of Disulfide Complex 2 (B), and tandem MS spectra of Disulfide Complex 2 (single and double charged species) (C). 
A.

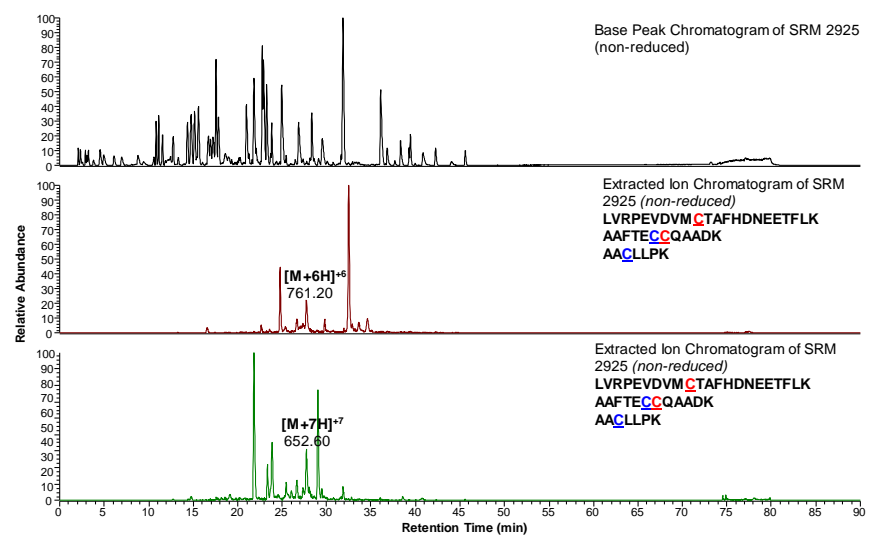

B.

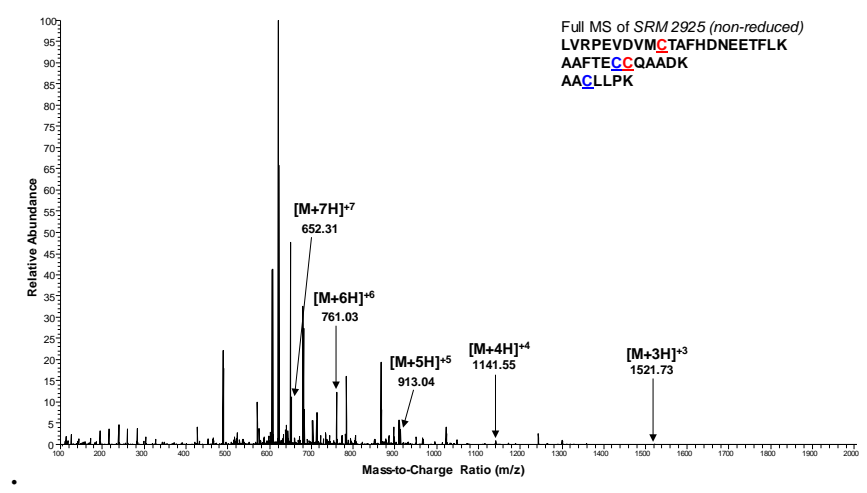

P1 Lㄴ.

P2 A]A]F[T]E]CC]C[Q]A]A]D]K

C.

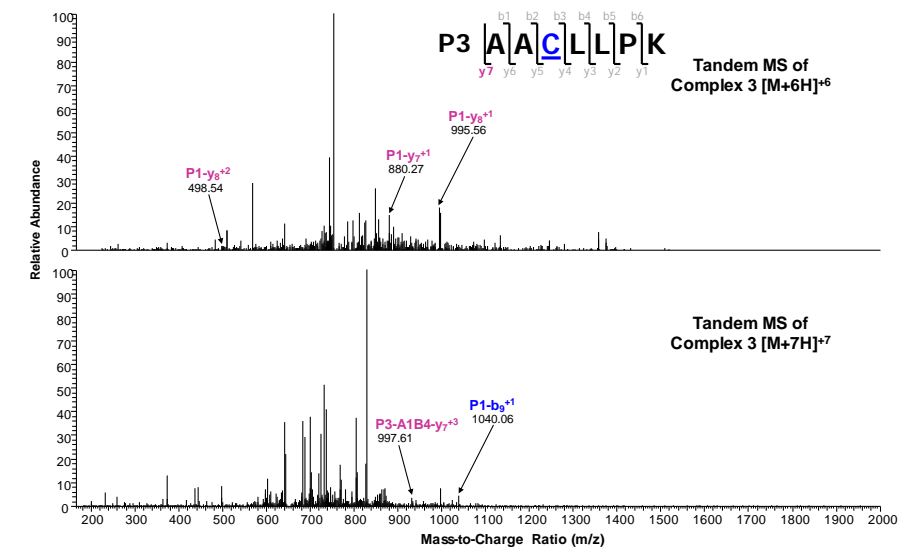

Figure F8. Disulfide profile of HSA Disulfide Complex 3 (see Figure 2) under the non-reduced condition. Base peak chromatogram and extracted-ion chromatograms (XICs) of tandem MS analysis of Disulfide Complex 3 (A), full MS spectrum of Disulfide Complex 3 (B), and tandem MS spectra of Disulfide Complex 3 (single and double charged species) (C). 

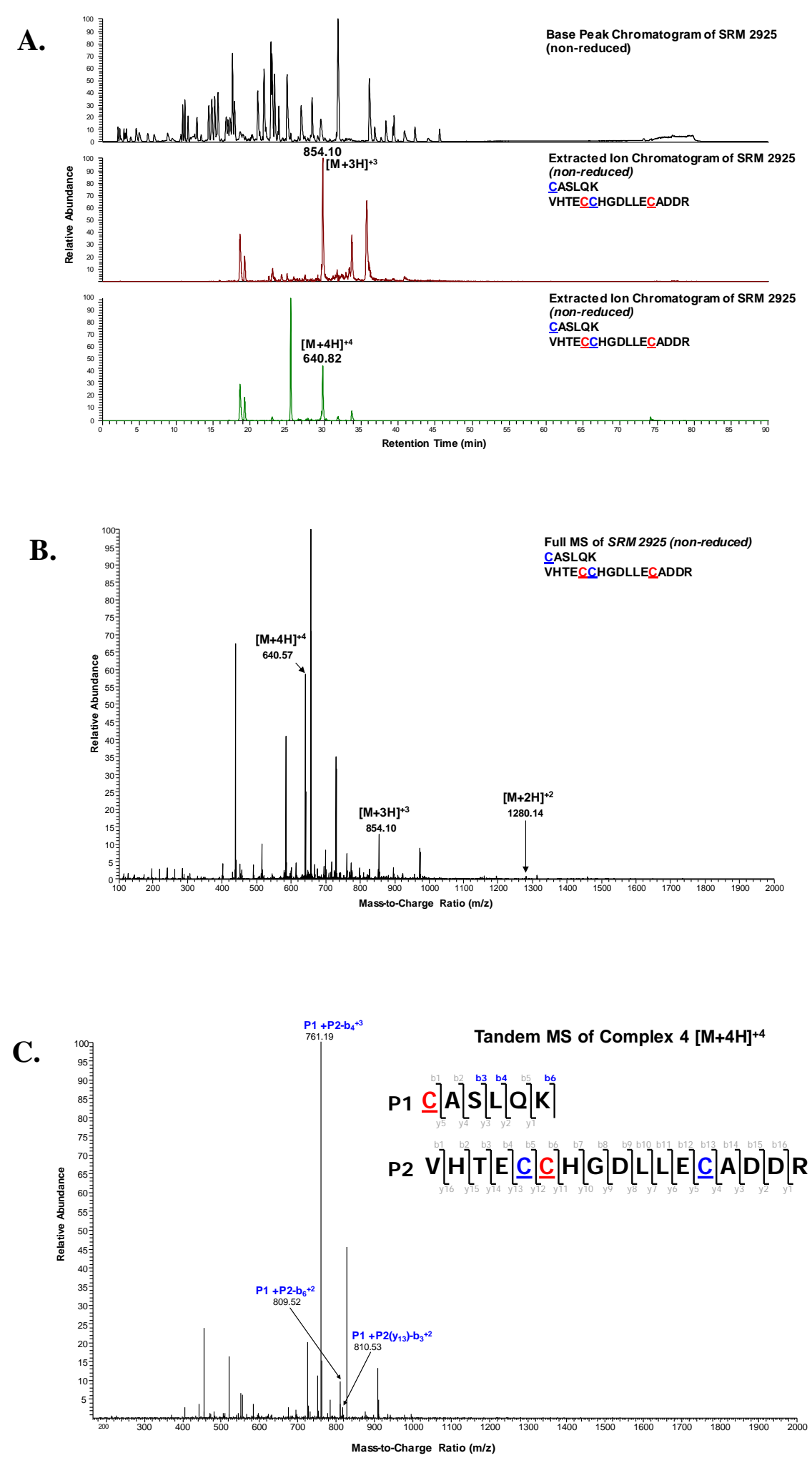

Figure F9. Disulfide profile of HSA Disulfide Complex 4 (see Figure 2) under the non-reduced condition. Base peak chromatogram and extracted-ion chromatograms (XICs) of tandem MS analysis of Disulfide Complex 4 (A), full MS spectrum of Disulfide Complex 4 (B), and tandem MS spectra of Disulfide Complex 4 (single and double charged species) (C). 

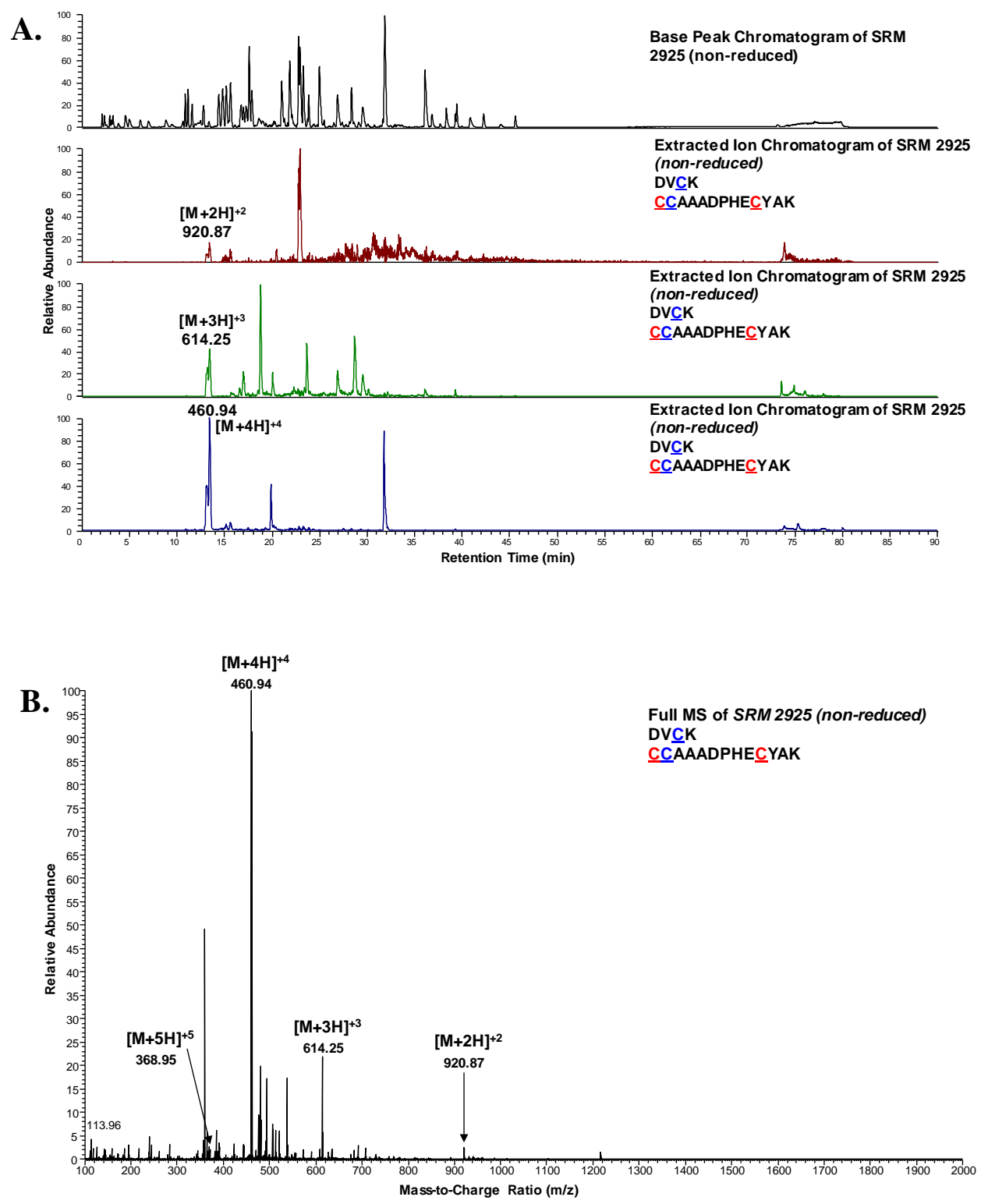

Figure F10. Disulfide profile of HSA Disulfide Complex 6 (see Figure 2) under the non-reduced condition. Base peak chromatogram and extracted-ion chromatograms (XICs) of tandem MS analysis of Disulfide Complex 6 (A) and the full MS spectra of Disulfide Complex 6 (B). 
A.
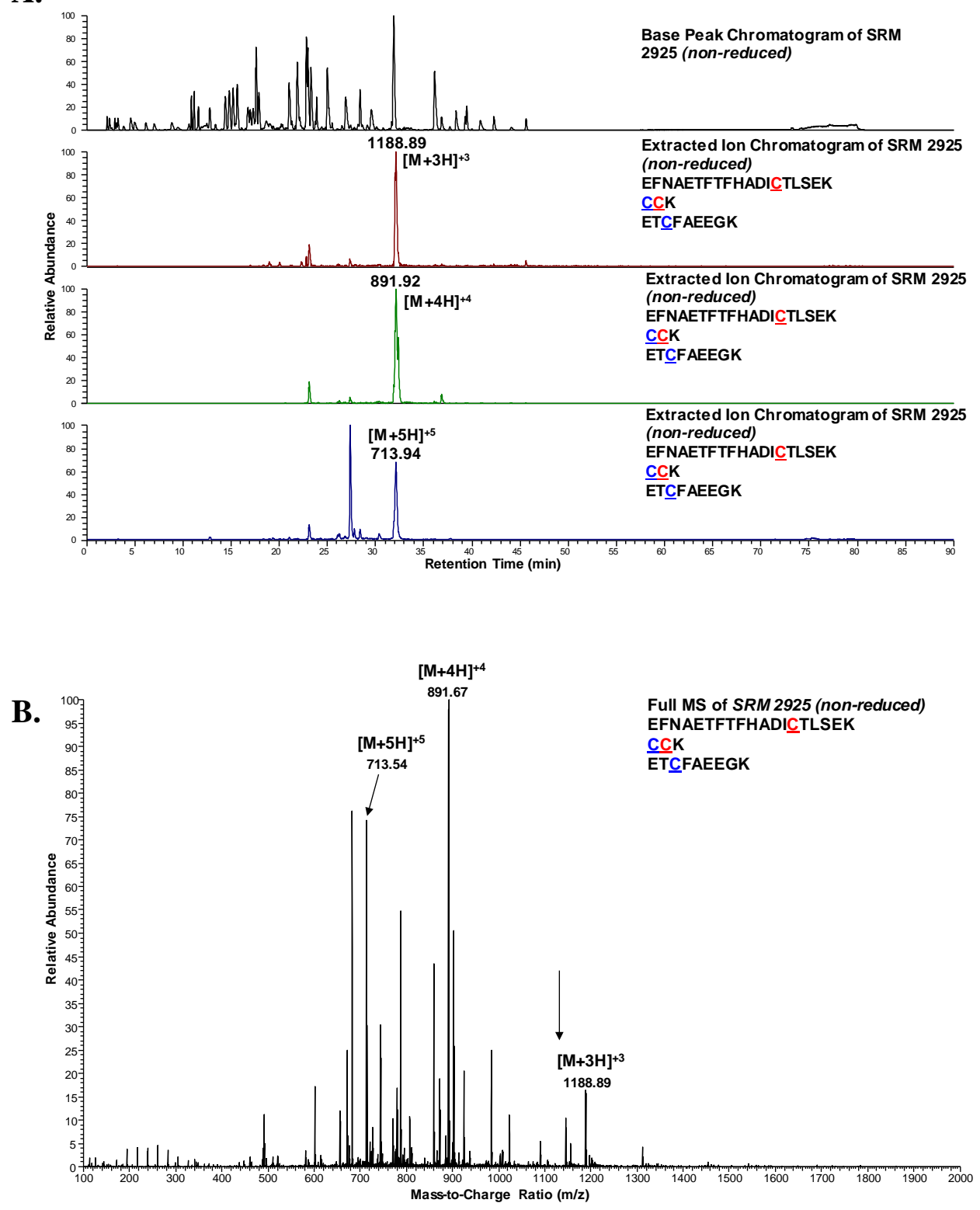

Figure F11. Disulfide profile of HSA Disulfide Complex 8 (see Figure 2) under the non-reduced condition. Base peak chromatogram and extracted-ion chromatograms (XICs) of tandem MS analysis of Disulfide Complex 8 (A) and the full MS spectra of Disulfide Complex 8 (B). 
Appendix F1. Peptide identifications of recombinant HSA in SRM 2925 from tandem MS analysis of trypsin digest products from the reduced experimental condition. The three randomly selected vials of SRM 2925 (process replicates; D1, D2, and D3) were digested and analyzed in quadruplicate (technical replicates, 1-4). (green: high confidence peptide identifications, yellow: medium confidence peptide identifications, red: low confidence peptide identifications, and gray: unidentified peptides)

\begin{tabular}{|c|c|}
\hline Sequence & $\begin{array}{c}\text { XCorr } \\
\text { Sequest HT }\end{array}$ \\
\hline$\overline{\text { AACLLPK }}$ & 2.74 \\
\hline AAFTECCQAADK & 4.03 \\
\hline ADLAK & 1.73 \\
\hline AEFAEVSK & 2.60 \\
\hline ALVLIAFAQYLQQCPFEDHVK & 6.43 \\
\hline AVMDDFAAFVEK & 4.19 \\
\hline AVMDDFAAFVEK & 3.82 \\
\hline AWAVAR & 1.98 \\
\hline CASLQK & 2.34 \\
\hline CCAAADPHECYAK & 4.81 \\
\hline \begin{tabular}{|l|} 
CCTESLVNR \\
\end{tabular} & 3.38 \\
\hline DDNPNLPR & 2.89 \\
\hline \begin{tabular}{|l} 
DLGEENFK \\
\end{tabular} & 3.08 \\
\hline DVFLGMFLYEYAR & 5.12 \\
\hline DVFLGMFLYEYAR & 5.11 \\
\hline ECCEKPLLEK & 2.94 \\
\hline EFNAETFTFHADICTLSEK & 6.91 \\
\hline ETCFAEEGK & 2.59 \\
\hline ETYGEMADCCAK & 3.75 \\
\hline ETYGEMADCCAK & 3.53 \\
\hline FQNALLVR & 3.14 \\
\hline HPDYSVVLLLR & 4.06 \\
\hline HPYFYAPELLFFAK & 4.53 \\
\hline LCTVATLR & 2.50 \\
\hline LDELR & 1.90 \\
\hline $\begin{array}{l}\text { LVAASQAALGL } \\
\end{array}$ & 3.77 \\
\hline LVNEVTEFAK & 3.68 \\
\hline LVRPEVDVMCTAFHDNEETFLK & 7.76 \\
\hline LVRPEVDVMCTAFHDNEETFLK & 4.62 \\
\hline LVTDLTK & 2.36 \\
\hline $\begin{array}{l}\text { MPCAEDYLSVVLNQLCVLHEK } \\
\end{array}$ & 7.01 \\
\hline \begin{tabular}{|l|} 
MPCAEDYLSVVLNQLCVLHEK \\
\end{tabular} & 6.93 \\
\hline NECFLQHK & 2.89 \\
\hline NYAEAK & 2.34 \\
\hline \begin{tabular}{|l|} 
QNCELFEQLGEYK \\
\end{tabular} & 5.07 \\
\hline QTALVELVK & 1.96 \\
\hline \begin{tabular}{|l} 
RPCFSALEVDETYVPK \\
\end{tabular} & 4.89 \\
\hline SEVAHR & 2.51 \\
\hline SHCIAEVENDEMPADLPSLAADFVESK & 8.29 \\
\hline SHCIAEVENDEMPADLPSLAADFVESK & 6.77 \\
\hline SLHTLFGDK & 3.15 \\
\hline TCVADESAENCDK & 4.74 \\
\hline TPVSDR & 1.77 \\
\hline TYETTLEK & 2.42 \\
\hline \begin{tabular}{|l} 
VFDEFKPLVEEPQNLIK \\
\end{tabular} & 5.52 \\
\hline $\begin{array}{l}\text { VHTECCHGDLLECADDR } \\
\end{array}$ & 6.77 \\
\hline VPQVSTPTLVEVSR & 4.40 \\
\hline YICENQDSISSK & 4.20 \\
\hline YLYEIAR & 2.71 \\
\hline DAHK & 0.28 \\
\hline DVCK & 1.48 \\
\hline EQLK & 1.30 \\
\hline FGER & 1.41 \\
\hline HPEAK & 1.92 \\
\hline LSQR & 1.29 \\
\hline NLGK & 1.30 \\
\hline QEPER & 1.17 \\
\hline \begin{tabular}{|l} 
ADDKETCFAEEGK \\
\end{tabular} & 4.37 \\
\hline AEFAEVSKLVTDLTK & 3.20 \\
\hline ATKEQLK & 2.67 \\
\hline DLGEENFKALVLIAFAQYLQQCPFEDHVK & 8.79 \\
\hline ETCFAEEGKK & 2.28 \\
\hline FKDLGEENFK & 4.31 \\
\hline HPYFYAPELLFFAKR & 4.00 \\
\hline KLVAASQAALGL & 3.54 \\
\hline KQTALVELVK & 4.49 \\
\hline $\begin{array}{l}\text { KVPQVSTPTLVEVSR } \\
\end{array}$ & 5.62 \\
\hline KYLYEIAR & 3.43 \\
\hline LDELRDEGK & 3.03 \\
\hline LKECCEKPLLEK & 4.86 \\
\hline $\begin{array}{l}\text { LSQRFPK } \\
\end{array}$ & 2.36 \\
\hline LVRPEVDVMCTAFHDNEETFLKK & 4.95 \\
\hline $\begin{array}{l}\text { MPCAEDYLSVVLNQLCVLHEKTPVSDR } \\
\end{array}$ & 5.71 \\
\hline NYAEAKDVFLGMFLYEYAR & 6.28 \\
\hline QEPERNECFLQHK & 2.88 \\
\hline QNCELFEQLGEYKFQNALLVR & 4.62 \\
\hline RHPDYSVVLLLR & 5.68 \\
\hline RHPYFYAPELLFFAK & 5.21 \\
\hline RMPCAEDYLSVVLNQLCVLHEK & 3.52 \\
\hline \begin{tabular}{|l} 
SLHTLFGDKLCTVATLR \\
\end{tabular} & 3.35 \\
\hline TCVADESAENCDKSLHTLFGDK & 3.93 \\
\hline VHTECCHGDLLECADDRADLAK & 3.14 \\
\hline \begin{tabular}{|l|} 
AAFTECCQAADKAACLLPK \\
\end{tabular} & 0.19 \\
\hline AWAVARLSQR & 0.66 \\
\hline ERQIK & 0.15 \\
\hline QIKK & 0.75 \\
\hline YTKK & 0.02 \\
\hline \begin{tabular}{|l} 
ADDKETCFAEEGKK \\
\end{tabular} & 5.93 \\
\hline AEFAEVSKLVTDLTKVHTECCHGDLLECADDR & 3.18 \\
\hline \begin{tabular}{|l|} 
DVCKNYAEAKDVFLGMFLYEYAR \\
\end{tabular} & 3.79 \\
\hline \begin{tabular}{|l|l|} 
FKDLGEENFKALVLIAFAQYLQQCPFEDHVK \\
\end{tabular} & 8.49 \\
\hline HPDYSVVLLLRLAKTYETTLEK & 3.88 \\
\hline $\begin{array}{l}\text { NYAEAKDVFLGMFLYEYARR } \\
\end{array}$ & 3.25 \\
\hline \begin{tabular}{|l} 
QNCELFEQLGEYKFQNALLVRYTK \\
\end{tabular} & 3.89 \\
\hline \begin{tabular}{|l|} 
RHPDYSVVLLLRLAK \\
\end{tabular} & 3.90 \\
\hline VGSKCCKHPEAK & 0.98 \\
\hline
\end{tabular}


Appendix F2. Peptide identifications of recombinant HSA in SRM 2925 from tandem MS analysis of trypsin digest products from the non-reduced experimental condition. The three randomly selected vials of SRM 2925 (process replicates; D4, D5, and D6) were digested and analyzed in quadruplicate (technical replicates, 1-4). (green: high confidence peptide identifications, yellow: medium confidence peptide identifications, red: low confidence peptide identifications, and gray: unidentified peptides)

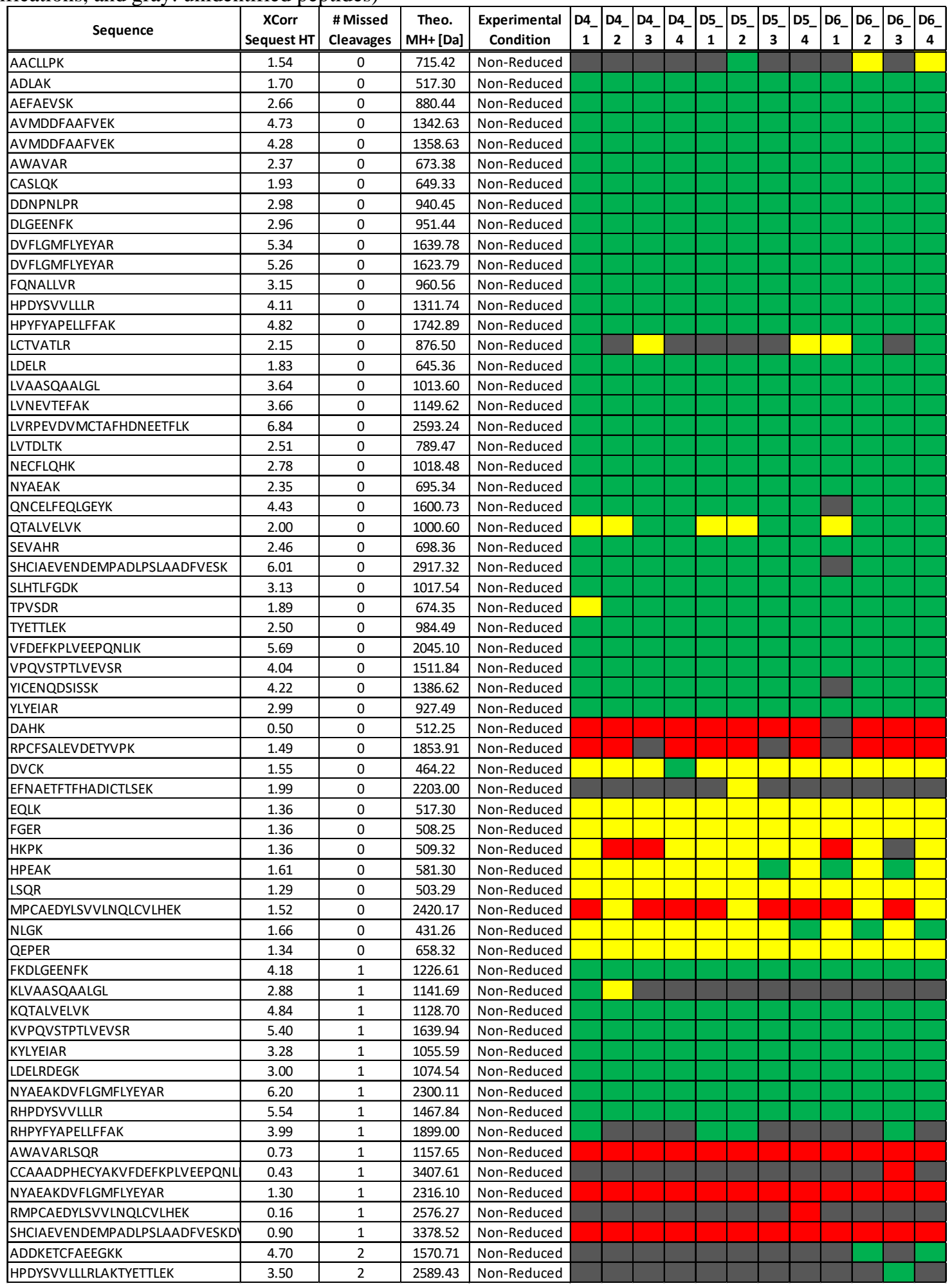


Appendix F3. Peptide identifications of recombinant HSA in SRM 2925 from tandem MS analysis of trypsin digest products identified in reduced (R), non-reduced (NR), or both (B) experimental conditions. (PC: Peptide Confidence Score, green: high confidence peptide identifications, yellow: medium confidence peptide identifications, red: low confidence peptide identifications, and gray: unidentified peptides)

\begin{tabular}{|c|c|c|c|c|c|c|}
\hline Sequence & $\begin{array}{l}\text { \# Missed } \\
\text { Cleavages }\end{array}$ & $\begin{array}{c}\text { Theo. } \\
\mathrm{MH}+[\mathrm{Da}]\end{array}$ & B & $\mathbf{R}$ & NR & PC \\
\hline AACLLPK & 0 & 772.44 & & & & \\
\hline AAFTECCQAADK & 0 & 1371.57 & & & & \\
\hline ADLAK & 0 & 517.30 & & & & \\
\hline AEFAEVSK & 0 & 880.44 & & & & \\
\hline ALVLIAFAQYLQQCPFEDHVK & 0 & 2490.28 & & & & \\
\hline AVMDDFAAFVEK & 0 & 1358.63 & & & & \\
\hline AVMDDFAAFVEK & 0 & 1342.63 & & & & \\
\hline AWAVAR & 0 & 673.38 & & & & \\
\hline CASLQK & 0 & 706.36 & & & & \\
\hline CCAAADPHECYAK & 0 & 1552.60 & & & & \\
\hline CCTESLVNR & 0 & 1138.50 & & & & \\
\hline DAHK & 0 & 470.24 & & & & \\
\hline DDNPNLPR & 0 & 940.45 & & & & \\
\hline DLGEENFK & 0 & 951.44 & & & & \\
\hline DVCK & 0 & 521.24 & & & & \\
\hline DVFLGMFLYEYAR & 0 & 1639.78 & & & & \\
\hline DVFLGMFLYEYAR & 0 & 1623.79 & & & & \\
\hline ECCEKPLLEK & 0 & 1305.62 & & & & \\
\hline EFNAETFTFHADICTLSEK & 0 & 2203.00 & & & & \\
\hline EFNAETFTFHADICTLSEK & 0 & 2260.02 & & & & \\
\hline EQLK & 0 & 517.30 & & & & \\
\hline ETCFAEEGK & 0 & 1070.45 & & & & \\
\hline ETYGEMADCCAK & 0 & 1434.53 & & & & \\
\hline ETYGEMADCCAK & 0 & 1450.53 & & & & \\
\hline FGER & 0 & 508.25 & & & & \\
\hline FQNALLVR & 0 & 960.56 & & & & \\
\hline HKPK & 0 & 509.32 & & & & \\
\hline HPDYSVVLLLR & 0 & 1311.74 & & & & \\
\hline HPEAK & 0 & 581.30 & & & & \\
\hline HPYFYAPELLFFAK & 0 & 1742.89 & & & & \\
\hline LCTVATLR & 0 & 933.52 & & & & \\
\hline LDELR & 0 & 645.36 & & & & \\
\hline LSQR & 0 & 503.29 & & & & \\
\hline LVAASQAALGL & 0 & 1013.60 & & & & \\
\hline LVNEVTEFAK & 0 & 1149.62 & & & & \\
\hline LVRPEVDVMCTAFHDNEETFLK & 0 & 2650.26 & & & & \\
\hline LVRPEVDVMCTAFHDNEETFLK & 0 & 2666.26 & & & & \\
\hline LVTDLTK & 0 & 789.47 & & & & \\
\hline MPCAEDYLSVVLNQLCVLHEK & 0 & 2420.17 & & & & \\
\hline MPCAEDYLSVVLNQLCVLHEK & 0 & 2534.21 & & & & \\
\hline MPCAEDYLSVVLNQLCVLHEK & 0 & 2518.21 & & & & \\
\hline NECFLQHK & 0 & 1075.50 & & & & \\
\hline NLGK & 0 & 431.26 & & & & \\
\hline NYAEAK & 0 & 695.34 & & & & \\
\hline QEPER & 0 & 658.32 & & & & \\
\hline QNCELFEQLGEYK & 0 & 1657.75 & & & & \\
\hline QTALVELVK & 0 & 1000.60 & & & & \\
\hline RPCFSALEVDETYVPK & 0 & 1853.91 & & & & \\
\hline
\end{tabular}




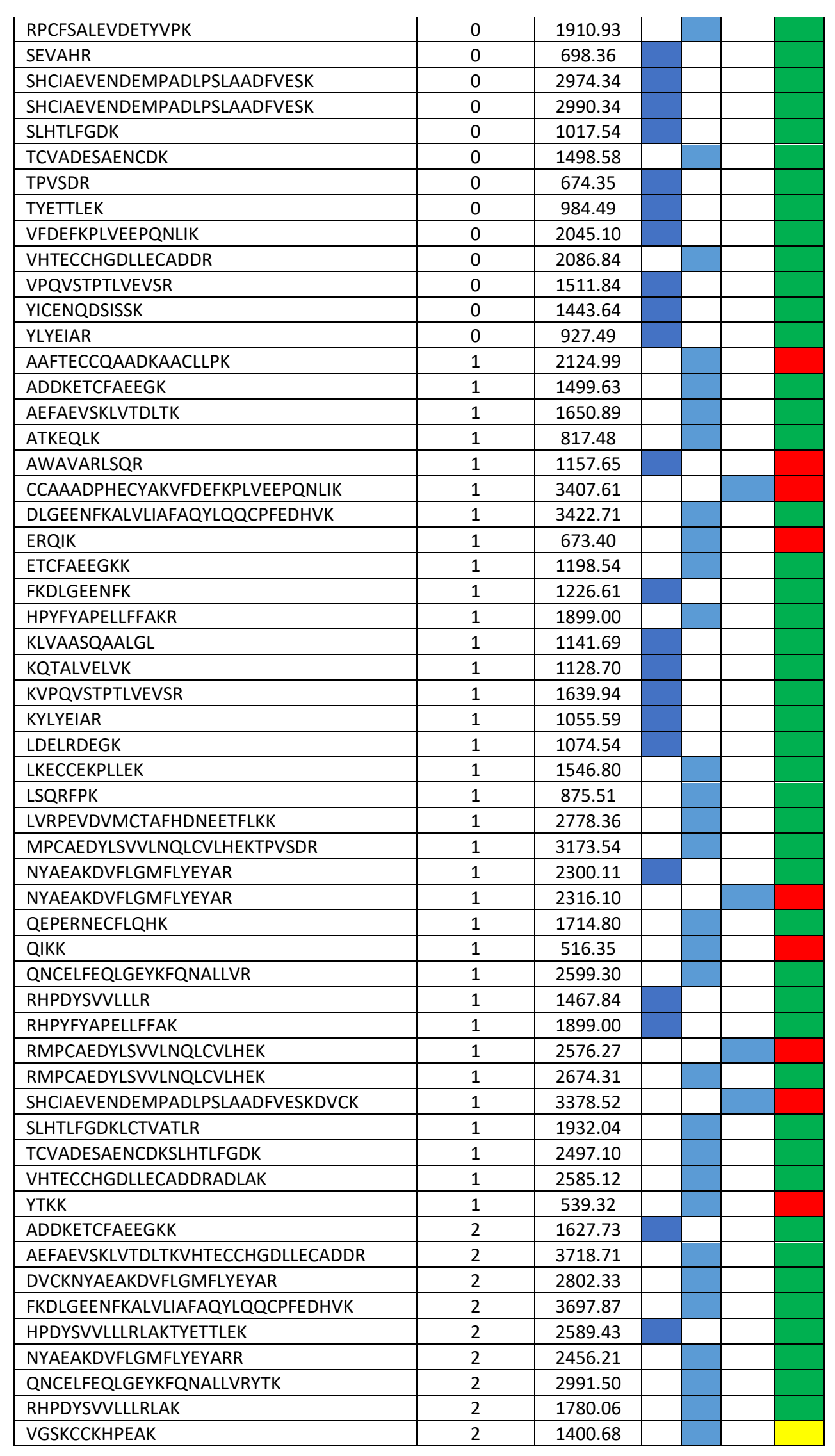




\section{APPENDIX G: Qualitative Characterization of SRM 2925 via Tandem Mass Spectrometry and 1-Dimensional Gel Electrophoresis}

\section{INTRODUCTION}

This report describes the qualitative characterization of recombinant HSA in SRM 2925 via the peptide profiling (tandem MS analysis) and 1-dimensional gel electrophoresis. Gel electrophoresis (polyacrylamide) can be a useful tool to investigate intra- and intermolecular disulfide linkages, which can provide insight to the conformational properties of proteins. [G1]

\section{EXPERIMENTAL}

Materials. NIST SRM 2925 Recombinant Human Serum Albumin (Primary Reference Calibrator for Urine Albumin) (Frozen), SRM 927e Bovine Serum Albumin (7 \% Solution), and SRM 927f Bovine Serum Albumin (7 \% Solution). Trypsin-Gold, MS-grade (Promega, Madison, WI, USA), Chymotrypsin, Sequencing-grade (Promega, Madison, WI, USA), high-purity LC-MS grade water/ $0.1 \%$ (v/v) formic acid and ACN/ $0.1 \%$ (v/v) formic acid (Honeywell Burdick and Jackson). The following were obtained from ThermoFisher Scientific: Imperial Protein Stain (PN: 24615), NuPAGE 4-12 \% Bis-Tris Mini Gel (PNNP0322BOX), NuPAGE 20X MES SDS Running Buffer (PN: NP0001), PageRuler Plus Pre-stained Protein Ladder (PN: 26619), NuPAGE 4X LDS Sample Buffer (PN-NP0008), NuPAGE Sample Reducing Agent (PN: NP0004), NuPAGE Antioxidant (PN-NP0005), Mini Gel Tank (PN: A26977), and Gel Loading Tops (PN-LC1001).

\section{Tandem MS Analysis}

In-solution Proteolytic Digestion. Each sample was incubated at $95^{\circ} \mathrm{C}$ for $10 \mathrm{~min}$ to denature HSA and cooled to room temperature (RT). For both the trypsin and chymotrypsin digest conditions, the samples were reduced with $5 \mathrm{mmol} / \mathrm{L}$ dithiothreitol at $60^{\circ} \mathrm{C}$ for $30 \mathrm{~min}$ followed by alkylation with $15 \mathrm{mmol} / \mathrm{L}$ iodoacetamide at RT for $30 \mathrm{~min}$ in the dark. For the trypsin digest, a 1:30 mass ratio of trypsin-to-protein was used for digestion at $37^{\circ} \mathrm{C}$ overnight $(\approx 18 \mathrm{~h})$ and for the chymotrypsin digest condition, a 1:25 mass ratio of chymotrypsin-to-protein was used for digestion at RT overnight ( $\approx 18 \mathrm{~h})$. Following the digestion, the $\mathrm{pH}$ of the sample was reduced with $50 \mathrm{~mL} / \mathrm{L}$ formic acid in water and incubated for $45 \mathrm{~min}$ at $37^{\circ} \mathrm{C}$ to quench the digestion reaction. The samples were concentrated and resuspended in $100 \mu \mathrm{L}$ of $0.1 \%(\mathrm{v} / \mathrm{v})$ formic acid in water for tandem MS analysis.

Liquid Chromatography-Tandem MS Analysis. LC general conditions: Dionex UltiMate 3000 HPLC System (Thermo Fisher; San Jose, CA) using an Agilent Zorbax 300SB-C18 column (2.1 mm x 150 mm, $3.5 \mu \mathrm{m})$. The column temperature was maintained at $40{ }^{\circ} \mathrm{C}$ and the peptides were loaded onto the column with a flowrate of $200 \mu \mathrm{L} / \mathrm{min}$ in $97 \%$ (v/v) Solvent A (water with $1 \mathrm{~mL} / \mathrm{L}$ formic acid) and $3 \%$ (v/v) Solvent B (acetonitrile with $1 \mathrm{~mL} / \mathrm{L}$ formic acid). The peptides were eluted from the stationary phase with a linear gradient and the total gradient time was 90 min. Solvent B was held constant at $3 \%$ for 5 min, ramped to $10 \%$ in $3 \mathrm{~min}$, ramped to $60 \%$ in $60 \mathrm{~min}$, and increased to $70 \%$ in $2 \mathrm{~min}$. At $72 \mathrm{~min}$, Solvent B was increased to $97 \%$ and held constant for 5 min and ramped down to $3 \%$ in 2 min for a 11-min column re-equilibration. Clean and blank runs preceded each analysis set to prevent carry-over.

General conditions for the LTQ Orbitrap Elite ETD (Thermo Fisher; San Jose, CA) linear ion trap mass spectrometer for CID in positive ion mode: Heated ESI, source heater temperature of $250{ }^{\circ} \mathrm{C}$, sheath gas flow rate of 35 (arbitrary units), auxiliary gas flow rate of 10 (arbitrary units), capillary temperature of 350 ${ }^{\circ} \mathrm{C}$, source voltage of $3.80 \mathrm{kV}$, S-lens RF level of $60 \%$, normalized collision energy of $35.0 \%$, default charge state of 2, activation q-value of 0.25 , and activation time at $10 \mathrm{~ms}$. 
The Top 4 data-dependent acquisition (DDA) experiment was used in this study, where the top four (4) most intense ions from the full MS (MS1) scan were targeted for MS/MS (MS2) analysis. The raw tandem MS data was processed manually and with the Protein Discoverer software (Version 2.3, Thermo Fisher), the UniProtKB/Swiss-Prot human serum albumin (P02768) fasta from http://uniprot.org- last modified on April 1, 1990 and the UniProtKB/Swiss-Prot bovine serum albumin (P02769) fasta from http://uniprot.orglast modified on February 1, 1996, the fasta files were used in the MS/MS search parameters.

A detailed description of the instrument conditions for the LC-MS system is outlined in Table 1 and Table G2.

\section{1-Dimensional Gel Electrophoresis}

1-D Gel Analysis. The protein samples are outlined in Table G3. For the reducing and non-reducing sample conditions, the intact protein samples were prepared according the manufacturer's specifications for the NuPAGE Gel Electrophoresis System. The samples were heated at $70{ }^{\circ} \mathrm{C}$ for $10 \mathrm{~min}$. and $10 \mu \mathrm{L}$ of each sample was added to the gels (well identification outlined in Table G4). Each gel (reduced and non-reduced) were run under the following conditions: Constant Voltage- $200 \mathrm{~V}$, Current- $500 \mathrm{~mA}$, and Run Time- 30 min. The proteins were visualized via Coomassie stain (Imperial Protein Stain) according to the manufacturer's specifications. Briefly, the gels were washed with $50 \mathrm{~mL}$ ultrapure water for $5 \mathrm{~min}$ at room temperature (repeated 4X), followed by a 10-min incubation ( $>12 \mathrm{ng}$ of protein material) with $20 \mathrm{~mL}$ Imperial Protein Stain at room temperature with gently orbital shaking. The stain solution was discarded, and the gel was washed with ultrapure water to reduce the background.

\section{RESULTS AND DISCUSSION}

Tandem MS Analysis. The amino acid sequence for recombinant HSA in SRM 2925 is presented in Figure G1, the sequence of the mature protein (amino acid residues 25-609; in bold) does not include the signal peptide and propeptide (amino acid residues 1-24; in gray), which is removed during normal posttranslational processing. According to the UniProt database, there are 17 disulfide bonds present in HSA between the following cysteine residues: 77-86, 99-115, 114-125, 148-193, 192-201, 224-270, 269-277, 289-303, 302-313, 340-385, 384-393, 416-462, 461-472, 485-501, 500-511, 538-583, and 582-591. From tandem MS analysis of three (3) vials of SRM 2925 (three technical replicates per vial), the sequence coverage observed for both trypsin and chymotrypsin digests is relatively high at $94 \%$ and $87 \%$, respectively (Figure G2). The base peak chromatograms for the trypsin and chymotrypsin digest conditions are shown in Figure G3 and G4, respectively. The peptide identifications are outlined in Appendix G1 for the trypsin digest condition and Appendix G2 for the chymotrypsin digest condition. The overlap in peptide identifications within-vial (technical replicates) and between-vial (process replicates) for both digest conditions (trypsin and chymotrypsin) is consistent with the repeatability of the base peak chromatograms observed for each run displayed in Figure G3 and G4. Therefore, we can determine from the high sequence coverage and peptide profile that the HSA protein in SRM 2925 is the mature form of the protein and the amino acid sequence is consistent with the theoretical sequence for HSA.

1-D Gel Electrophoresis Analysis. To assess the quality of SRM 2925, 1-D gel electrophoresis analysis was conducted to evaluate protein purity. NIST SRM 927e and SRM 927f were used as quality control materials to assess the performance of the gel system since the gels were run separately due to different sample conditions. In the reduced condition (Figure G5-A), a reducing agent (DTT) is added to the sample to disrupt the disulfide linkages [17 intramolecular disulfide linkages in native bovine serum albumin (BSA) and HSA] and under the non-reduced condition (Figure G5-B) no reducing agent is applied to the sample, maintaining the integrity of the disulfide linkages. The difference in the protein migration profile between the two gels is based on the presence/absence of the disulfide linkages (Figure G5-A and G5-B). In the reduced gel (Figure G5-A), the major band for the BSA materials (SRMs 927e and 927f) is consistent with the molecular weight (MW) of mature, reduced BSA (reduced and non-reduced MW for BSA is listed in 
Table G3) and the major band for SRM 2925 is consistent with the MW of mature, reduced HSA (reduced and non-reduced MW for HSA is listed in Table G3). The high MW bands present in the non-reduced condition for SRM 927f and SRM 2925 could be due to protein-protein interactions (i.e., protein dimers), possibly mediated by disulfide linkages on the free cysteine for both BSA and HSA. The higher MW bands in SRM 927e appear in both conditions, which could indicate the presence of protein-protein interactions not mediated by disulfide linkages, further investigation is required to determine the exact protein-protein interactions present in SRM 927e. The migration shift observed in the major band of each protein in the non-reduced gel, in comparison to the reduced gel, is a result of the difference in the 3-dimensional structure of the BSA/HSA molecule based on the presence of intramolecular disulfide linkages. Reduced and fully unfolded proteins migrate at a rate proportional to the MW; however, the presence of intramolecular disulfide bonds reduces the Stokes radius of the protein, which increases the gel migration rate. [G2, G3] This increased migration rate of non-reduced proteins, can lead to a band at a MW inconsistent with that of the protein. [G4] Therefore, the presence of the intramolecular disulfide linkages reduces the protein size and increases the migration speed, resulting in migration of the major band of all the proteins at a lower MW in the non-reduced gel compared to the reduced gel.

Overall, the protein profile observed via 1-dimensional gel analysis and the peptide profile observed via tandem MS analysis of the protein digest products of HSA support the use of SRM 2925 as a primary reference material for the determination of urine albumin in candidate NIST SRM 3666 Albumin and Creatinine in Frozen Human Urine.

\section{REFERENCES}

G1. Janatova J. "Detection of disulphide bonds and localization of interchain linkages in the third (C3) and fourth (C4) components of human complement.” Biochem J. 1986 Feb 1: 223(3): 819-825.

G2. Goldberger G, Creighton TE. "Gel electrophoresis in studies of protein conformation and folding." Anal Biochem. 1984 Apr: 138(1): 1-18.

G3. Griffith IP. "The effect of cross-links on the mobility of proteins in dodecyl sulphate-polyacrylamide gels.” Biochem J. 1972 Feb: 126(3): 553-560.

G4. Allore RJ, Barber BH. "Inter- and intramolecular disulfide bonding among lymphocyte plasma membrane proteins and glycoproteins.” Mol Immunol. 1983 Apr;20(4):383-395. 
Table G1. Liquid chromatography linear gradient.

\begin{tabular}{|c|c|c|}
\hline Time (min) & \% Solvent $^{\boldsymbol{a}}$ & $\mathbf{\% S o l v e n t ~}^{\boldsymbol{b}}$ \\
\hline 0 & 97 & 3 \\
\hline 5 & 97 & 3 \\
\hline 8 & 90 & 10 \\
\hline 68 & 40 & 60 \\
\hline 70 & 30 & 70 \\
\hline 72 & 3 & 97 \\
\hline 77 & 3 & 97 \\
\hline 79 & 97 & 3 \\
\hline 90 & 97 & 3 \\
\hline
\end{tabular}

${ }^{a}$ Solvent A: $0.1 \%$ formic acid in water

${ }^{b}$ Solvent B: $0.1 \%$ formic acid in acetonitrile

Table G2. Tandem MS instrument parameters.

\begin{tabular}{|c|c|c|}
\hline MS Type & Parameter & Value \\
\hline \multirow[t]{9}{*}{ MS Source } & Acquisition Delay Time (minutes) & $\overline{3.0}$ \\
\hline & Source Type & HESI \\
\hline & Capillary Temp $\left({ }^{\circ} \mathrm{C}\right)$ & 350 \\
\hline & Source Heater Temp $\left({ }^{\circ} \mathrm{C}\right)$ & 250 \\
\hline & Sheath Gas Flow & 35 \\
\hline & Aux Gas Flow & 10 \\
\hline & Sweep Gas Flow & 0.0 \\
\hline & Source Voltage (V) & 3.8 \\
\hline & S-lens RF Level (\%) & 67 \\
\hline \multirow[t]{4}{*}{ MS Global DDA $^{a}$} & Exclusion mass width-low & 0.5 \\
\hline & Exclusion mass width-high & 1.5 \\
\hline & Reject mass width-low & 0.5 \\
\hline & Reject mass width-high & 0.5 \\
\hline \multirow[t]{4}{*}{ Dynamic Exclusion $^{a}$} & Repeat Count & 3 \\
\hline & Repeat Duration (s) & 30 \\
\hline & Exclusion List Size & 500 \\
\hline & Exclusion Duration (s) & 20 \\
\hline \multirow[t]{5}{*}{ Scan Event } & "Activation Type & CID \\
\hline & Min. Signal Threshold & 500 \\
\hline & Normalized CE & 35 \\
\hline & Default Charge State & 2 \\
\hline & Activation Q & 0.250 \\
\hline
\end{tabular}

${ }^{a}$ DDA: Data-Dependent Acquisition 
Table G3. List of NIST Standard Reference Materials (SRMs) used in study.

\begin{tabular}{|c|c|c|c|c|c|c|c|c|}
\hline $\begin{array}{l}\text { Sample } \\
\text { Name }\end{array}$ & Protein & $\begin{array}{c}\text { UniProt } \\
\text { Accession \# }\end{array}$ & $\begin{array}{c}\text { Mature } \\
\text { Protein } \\
\text { (Residues) }\end{array}$ & $\begin{array}{c}\text { Expected } \\
\text { Molecular } \\
\text { Mass }^{a} \\
(\mathrm{~g} / \mathrm{mol}) \\
\end{array}$ & $\begin{array}{c}\text { Expected } \\
\text { Molecular } \\
\text { Mass }{ }^{b} \\
(\mathrm{~g} / \mathbf{m o l}) \\
\end{array}$ & $\begin{array}{c}\text { Stock Protein } \\
\text { Concentration } \\
\text { ( } \pm \text { Expanded } \\
\text { Uncertainty) } \\
\end{array}$ & $\begin{array}{c}\text { Working } \\
\text { Protein } \\
\text { Concentration }\end{array}$ & $\begin{array}{c}\text { Protein Amount } \\
\text { Loaded on Gel } \\
(\mu \mathrm{g}) \text { (reduced/non- } \\
\text { reduced) }\end{array}$ \\
\hline $\begin{array}{c}\text { NIST SRM } \\
\text { 927e }\end{array}$ & $\begin{array}{l}\text { Bovine Serum } \\
\text { Albumin (BSA) }\end{array}$ & P02769 & $25-607$ & $66,389.76$ & $66,355.49$ & $67.38 \mathrm{~g} / \mathrm{L} \pm 1.38 \mathrm{~g} / \mathrm{L}$ & $1.0 \mathrm{~g} / \mathrm{L}$ & 5.0 \\
\hline $\begin{array}{l}\text { NIST SRM } \\
\text { 927f }\end{array}$ & $\begin{array}{l}\text { Bovine Serum } \\
\text { Albumin (BSA) }\end{array}$ & P02769 & $25-607$ & $66,389.76$ & $66,355.49$ & $\approx 67.38 \mathrm{~g} / \mathrm{L}$ & $1.0 \mathrm{~g} / \mathrm{L}$ & 5.0 \\
\hline $\begin{array}{l}\text { NIST SRM } \\
2925\end{array}$ & $\begin{array}{c}\text { Recombinant } \\
\text { Human Serum } \\
\text { Albumin (HAS) }\end{array}$ & P02768 & $25-609$ & $66,428.83$ & $66,394.56$ & $0.958 \mathrm{~g} / \mathrm{L} \pm 0.022 \mathrm{~g} / \mathrm{L}$ & $0.958 \mathrm{~g} / \mathrm{L}$ & 4.8 \\
\hline
\end{tabular}

${ }^{a}$ Theoretical molecular mass of material (HSA and BSA) without inclusion of 17 disulfide bonds.

${ }^{b}$ Theoretical molecular mass of material (HSA and BSA) with inclusion of 17 disulfide bonds.

Table G4. Gel lane protein identification for each gel condition.

\begin{tabular}{|c|c|c|}
\hline Gel Lane Number & Reduced Condition & Non-Reduced Condition \\
\hline \hline $\mathbf{1}$ & Protein MW Ladder $(5 \mu \mathrm{L})$ & Protein MW Ladder $(5 \mu \mathrm{L})$ \\
\hline $\mathbf{2}$ & Blank & Blank \\
\hline $\mathbf{3}$ & SRM 927e (5.0 $\mathbf{\mu g})$ & SRM 927e (5.0 $\boldsymbol{\mu g})$ \\
\hline $\mathbf{4}$ & Blank & Blank \\
\hline $\mathbf{5}$ & SRM 927f (5.0 $\boldsymbol{\mu g})$ & SRM 927f (5.0 $\boldsymbol{\mu g})$ \\
\hline $\mathbf{6}$ & Blank & Protein MW Ladder $(5 \mu \mathrm{L})$ \\
\hline $\mathbf{7}$ & SRM 2925 (4.8 $\boldsymbol{\mu g})$ & SRM $2925 \mathbf{( 4 . 8} \boldsymbol{\mu g})$ \\
\hline $\mathbf{8}$ &
\end{tabular}

\begin{tabular}{|c|c|c|c|c|}
\hline 10 & 20 & 30 & 40 & 50 \\
\hline MKWVTFISLL & FLFSSAYSRG & VFRRDAHKSE & VAHRFKDLGE & ENFKALVLIA \\
\hline 60 & 70 & 80 & 90 & 100 \\
\hline FAQYLQQCPF & EDHVKLVNEV & TEFAKTCVAD & ESAENCDKSL & HTLFGDKLCT \\
\hline 110 & 120 & 130 & 140 & 150 \\
\hline VATLRETYGE & MADCCAKQEP & ERNECFLQHK & DDNPNLPRLV & RPEVDVMCTA \\
\hline 160 & 170 & 180 & 190 & 200 \\
\hline FHDNEETFLK & KYLYEIARRH & PYFYAPELLF & FAKRYKAAFT & ECCQAADKAA \\
\hline 210 & 220 & 230 & 240 & 250 \\
\hline CLLPKLDELR & DEGKASSAKQ & RLKCASLQKF & GERAFKAWAV & ARLSQRFPKA \\
\hline 260 & 270 & 280 & 290 & 300 \\
\hline EFAEVSKLVT & DLTKVHTECC & HGDLLECADD & RADLAKYICE & NQDSISSKLK \\
\hline 310 & 320 & 330 & 340 & 350 \\
\hline ECCEKPLLEK & SHCIAEVEND & EMPADLPSLA & ADFVESKDVC & KNYAEAKDVF \\
\hline 360 & 370 & 380 & 390 & 400 \\
\hline LGMFLYEYAR & RHPDYSVVLL & LRLAKTYETT & LEKCCAAADP & HECYAKVFDE \\
\hline 410 & 420 & 430 & 440 & 450 \\
\hline FKPLVEEPQN & LIKQNCELFE & QLGEYKFQNA & LLVRYTKKVP & QVSTPTLVEV \\
\hline 460 & 470 & 480 & 490 & 500 \\
\hline SRNLGKVGSK & CCKHPEAKRM & PCAEDYLSVV & LNQLCVLHEK & TPVSDRVTKC \\
\hline 510 & 520 & 530 & 540 & 550 \\
\hline CTESLVNRRP & CFSALEVDET & YVPKEFNAET & FTFHADICTL & SEKERQIKKQ \\
\hline 560 & 570 & 580 & 590 & 600 \\
\hline TALVELVKHK & PKATKEQLKA & AFVE & DDKET & EGKKLV \\
\hline
\end{tabular}

AASQAALGL

Figure G1. Amino acid sequence of HSA protein in SRM 2925 according to the UniProt database (P02768), including the 17 disulfide bonds between the following cysteine residues: 77-86, 99-115, 114125, 148-193, 192-201, 224-270, 269-277, 289-303, 302-313, 340-385, 384-393, 416-462, 461-472, 485$501,500-511,538-583$, and 582-591. The sequence of the mature protein (amino acid residues 25-609) is represented in bold and the signal peptide and propeptide (amino acid residues 1-24) are both represented in gray. 


\section{Trypsin Digest Condition}

DAHKSEVAHR FKDLGEENFK ALVLIAFAQY LQQCPFEDHV KLVNEVTEFA KTCVADESAE NCDKSLHTLF GDKLCTVATL RETYGEMADC CAKQEPERNE CFLQHKDDNP NLPRLVRPEV DVMCTAFHDN EETFLKKYLY EIARRHPYFY APELLFFAKR YKAAFTECCQ AADKAACLLP KLDELRDEGK ASSAKQRLKC ASLQKFGERA FKAWAVARLS QRFPKAEFAE VSKLVTDLTK VHTECCHGDL LECADDRADL AKYICENQDS ISSKLKECCE KPLLEKSHCI AEVENDEMPA DLPSLAADFV ESKDVCKNYA EAKDVFLGMF LYEYARRHPD YSVVLLLRLA KTYETTLEKC CAAADPHECY AKVFDEFKPL VEEPQNLIKQ NCELFEQLGE YKFQNALLVR YTKKVPQVST PTLVEVSRNL GKVGSKCCKH PEAKRMPCAE DYLSVVLNQL CVLHEKTPVS DRVTKCCTES LVNRRPCFSA LEVDETYVPK EFNAETFTFH ADICTLSEKE RQIKKQTALV ELVKHKPKAT KEQLKAVMDD FAAFVEKCCK ADDKETCFAE EGKKLVAASQ AALGL

\section{Chymotrypsin Digest Condition}

DAHKSEVAHR FKDLGEENFK ALVLIAFAQY LQQCPFEDHV KLVNEVTEFA KTCVADESAE NCDKSLHTLF GDKLCTVATL RETYGEMADC CAKQEPERNE CFLQHKDDNP NLPRLVRPEV DVMCTAFHDN EETFLIKKYLY EIARRHPYFY APELLFFAKR YKAAFTECCQ AADKAACLLP KLDELRDEGK ASSAKQRLKC ASLQKFGERA FKAWAVARLS QRFPKAEFAE VSKLVTDLTK VHTECCHGDL LECADDRADL AKYICENQDS ISSKLKECCE KPLLEKSHCI AEVENDEMPA DLPSLAADFV ESKDVCKNYA EAKDVFLGMF LYEYARRHPD YSVVLLLRLA KTYETTLEKC CAAADPHECY AKVFDEFKPL VEEPQNLIKQ NCELFEQLGE YKFQNALLVR YTKKVPQVST PTLVEVSRNL GKVGSKCCKH PEAKRMPCAE DYLSVVLNQL CVLHEKTPVS DRVTKCCTES

LVNRRPCFSA LEVDETYVPK EFNAETFTFH ADICTLSEKE RQIKKQTALV ELVKHKPKAT KEQLKAVMDD FAAFVEKCCK ADDKETCFAE EGKKLVAASQ AALGL

Figure G2. Protein sequence coverage for trypsin (94\%) and chymotrypsin (87\%) digest conditions. (green: high confidence peptide identifications, yellow: medium confidence peptide identifications, red: low confidence peptide identifications, and black: unidentified peptides) 

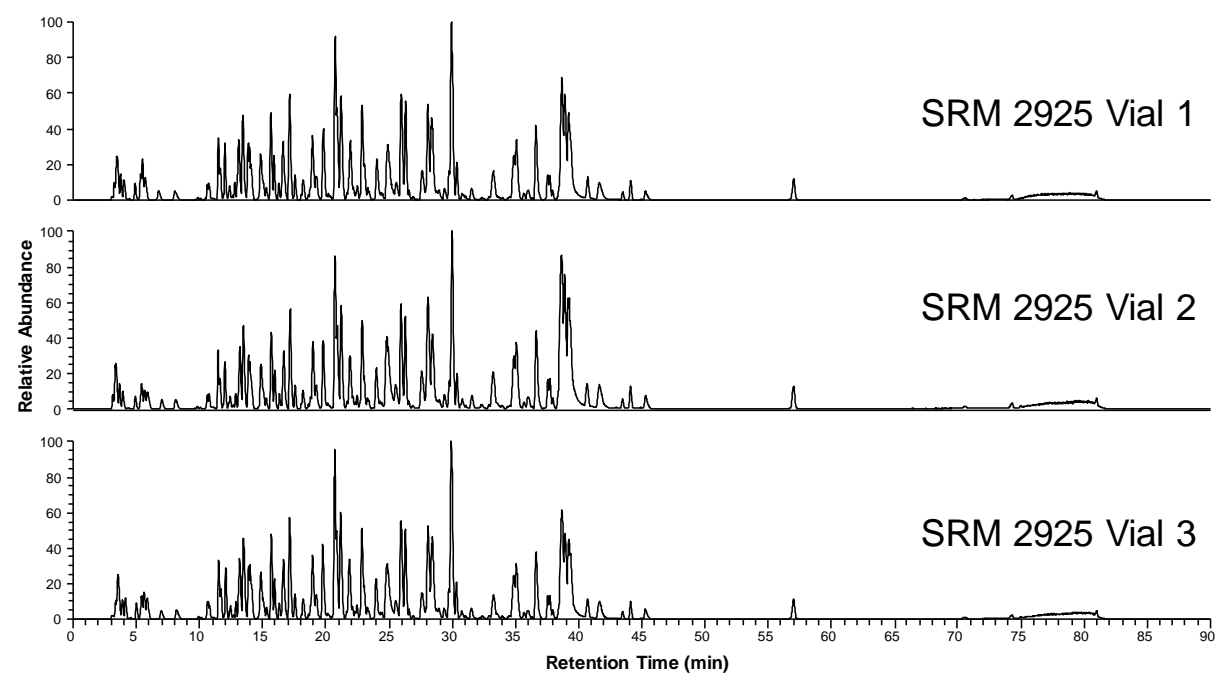

Figure G3-A. Base peak chromatograms for three process replicates (vials) of SRM 2925-trypsin digest condition.

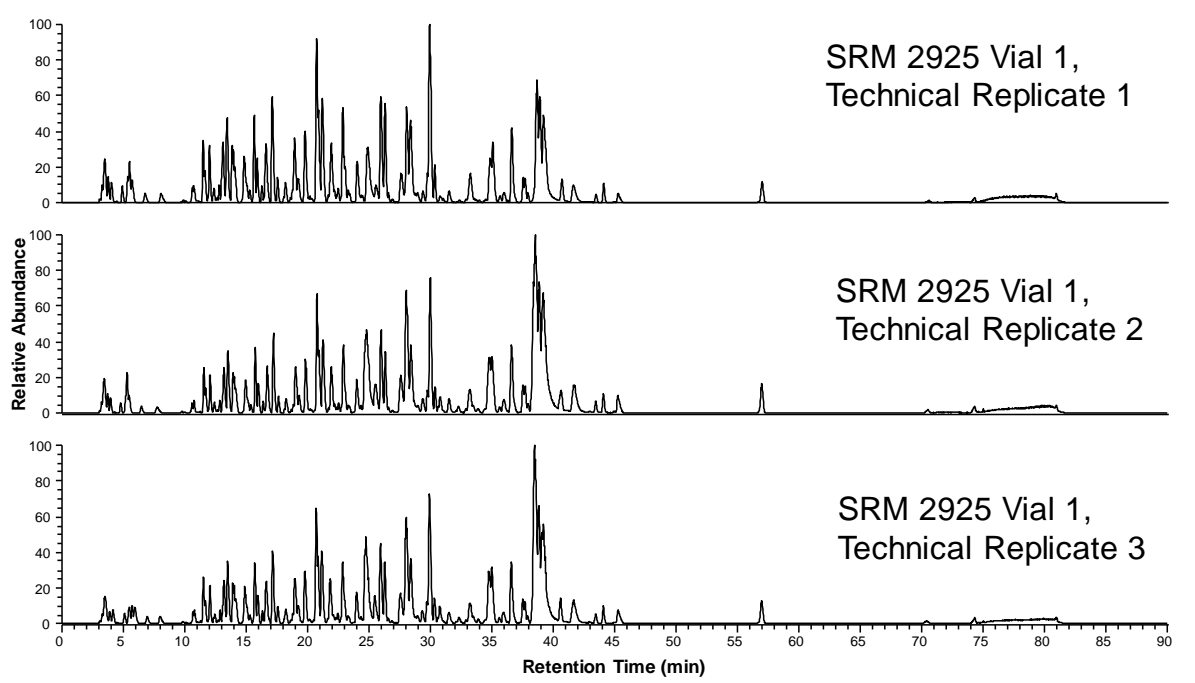

Figure G3-B. Base peak chromatograms for three technical replicates of one vial of SRM 2925- trypsin digest condition. 

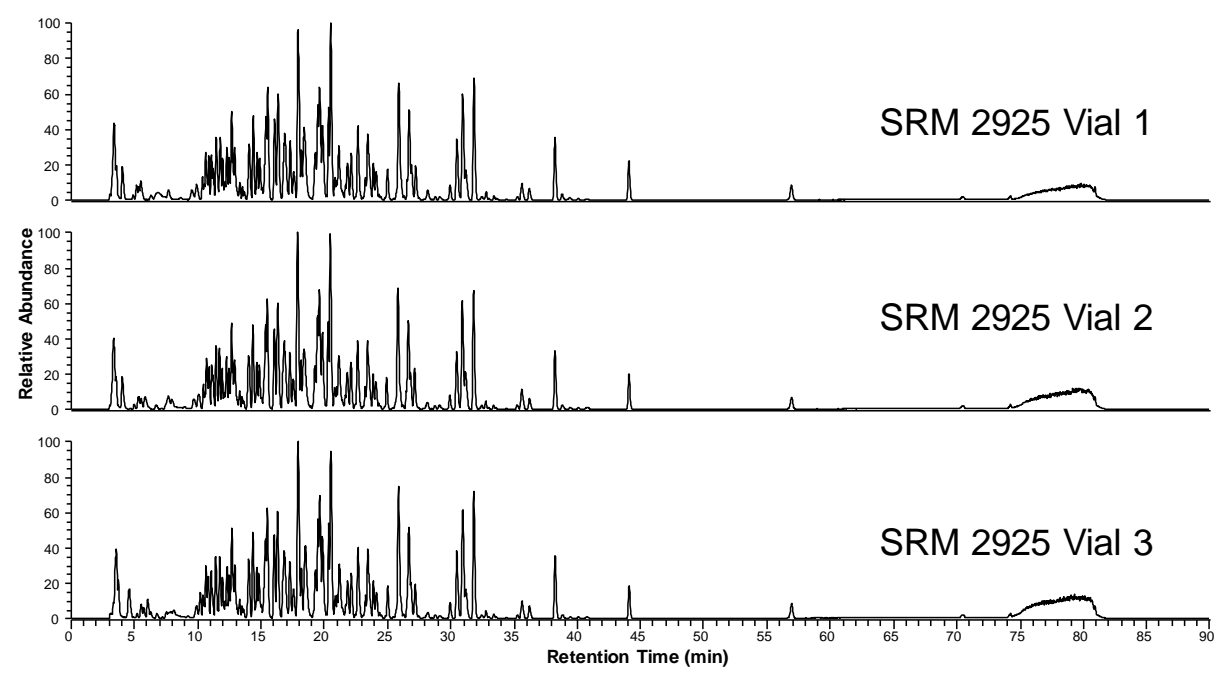

Figure G4-A. Base peak chromatograms for three process replicates (vials) of SRM 2925-chymotrypsin digest condition.
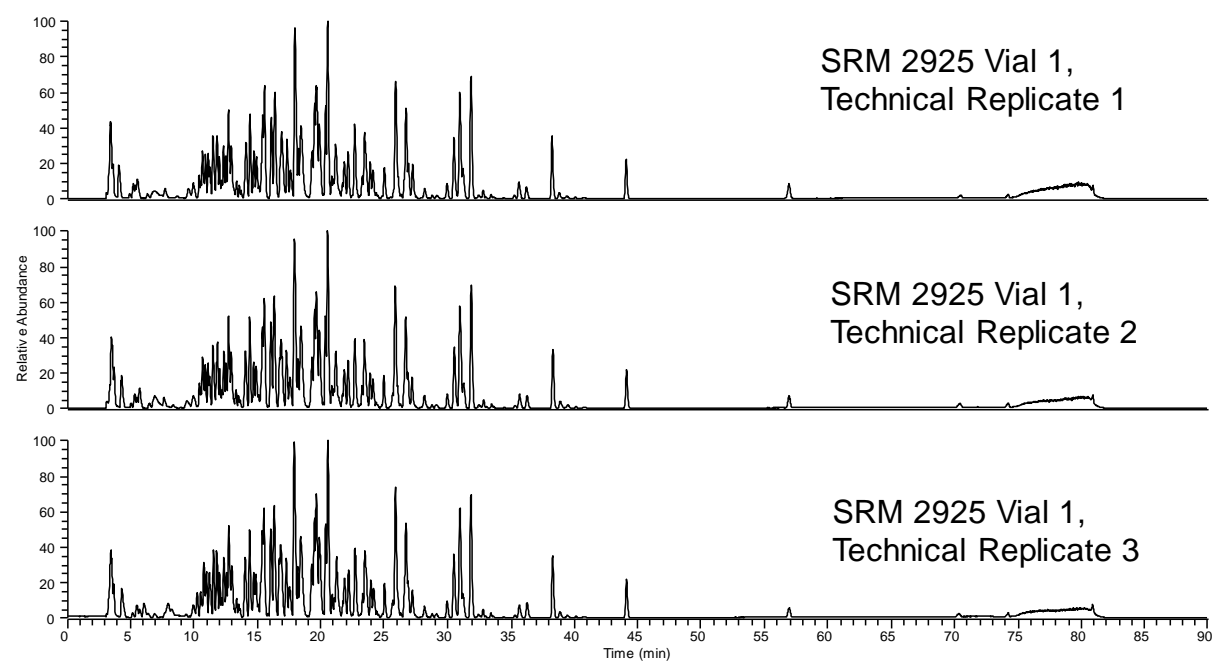

Figure G4B. Base peak chromatograms for three technical replicates of one vial of SRM 2925chymotrypsin digest condition. 


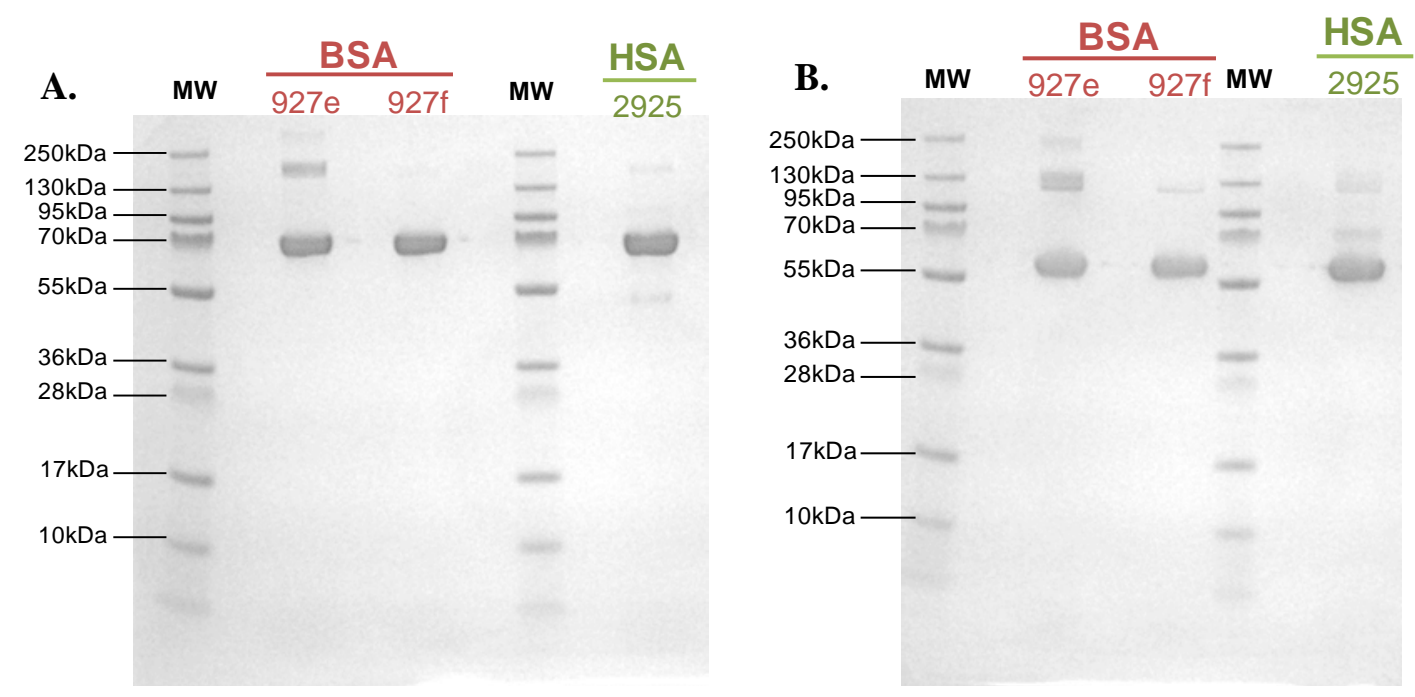

Figure G5. 1-Dimensional gel analysis of SRM 2925 under reduced (A) and non-reduced (B) conditions. (QC materials: SRM 927e and SRM 927f) 
Appendix G1. Peptide identifications of recombinant HSA in SRM 2925 from tandem MS analysis of trypsin digest products. The three randomly selected vials of SRM 2925 (process replicates, Vial 1-3) were digested and analyzed in triplicate (technical replicates, 1-3). (green: high confidence peptide identifications, yellow: medium confidence peptide identifications, red: low confidence peptide identifications, and black: unidentified peptides)

\begin{tabular}{|c|c|c|c|c|c|c|c|c|c|c|}
\hline & & & & & & & & & Vial 1 & \\
\hline Peptide Sequence & XCorr ${ }^{a}$ & $\begin{array}{c}\# \\
\text { PSMs }\end{array}$ & $\begin{array}{l}\mathrm{m} / \mathrm{z}[\mathrm{M}+\mathrm{H}]^{+1} \\
{[\mathrm{Da}]}\end{array}$ & $\mathbf{R T}^{b}[\mathrm{~min}]$ & Charge & $\begin{array}{l}\text { Identified } \\
\mathrm{m} / \mathrm{z}[\mathrm{Da}]\end{array}$ & $\begin{array}{l}\text { \# Missed } \\
\text { Cleavages }\end{array}$ & 1 & 2 & 3 \\
\hline LVRPEVDVMCTAFHDNEETFLK & 7.86 & $\frac{163}{163}$ & $\frac{1 \mathrm{Lal}}{2650.26}$ & 27.5 & 3 & 884.09 & Cleavages & & & \\
\hline $\begin{array}{l}\text { SHCIAEVENDEMPADLPSLAADFVESK } \\
\end{array}$ & 7.27 & 85 & 2990.34 & 30.7 & 3 & 997.45 & 0 & & & \\
\hline MPCAEDYLSVVLNQLCVLHEK & 7.12 & 290 & 2518.21 & 38.7 & 3 & 840.07 & 0 & & & \\
\hline SHCIAEVENDEMPADLPSLAADFVESK & $\frac{7.28}{6.84}$ & 280 & 2974.34 & 33.1 & $\frac{3}{4}$ & 744.34 & 0 & & & \\
\hline EFNAETFTFHADICTLSEK & 6.83 & 5100 & 2260.02 & 34.1 & 2 & 1130.51 & 0 & & & \\
\hline ALVLIAFAQYLQQCPFEDHVK & 6.82 & 8452 & 2490.28 & 50.6 & $\frac{2}{2}$ & 1245.65 & 0 & & & \\
\hline MPCAEDYLSVVLNQLCVLHEK & 6.75 & 36 & 2534.21 & 37.5 & 3 & 845.41 & 0 & & & \\
\hline VHTECCHGDLLECADDR & 6.27 & 322 & 2086.84 & 14.8 & 2 & 1043.92 & 0 & & & \\
\hline VFDEFKPLVEEPQNLIK & $\frac{0.27}{5.85}$ & $\frac{322}{264}$ & $\frac{2008.64}{2045.10}$ & $\frac{14.8}{30.2}$ & $\frac{2}{2}$ & $\frac{1043.92}{1023.05}$ & 0 & & & \\
\hline DVFLGMFLYEYAR & 5.25 & 5036 & 1623.79 & 41.3 & 3 & 541.93 & 0 & & & \\
\hline QNCELFEQLGEYK & 5.18 & 217 & 1657.75 & 25.9 & 3 & 553.26 & 0 & & & \\
\hline DVFLGMFLYEYAR & 5.02 & 186 & 1639.78 & 35.9 & 3 & 547.26 & 0 & & & \\
\hline RPCFSALEVDETYVPK & 5.02 & 344 & 1910.93 & $\frac{23.5}{23.6}$ & $\frac{5}{3}$ & $\frac{341.20}{637.65}$ & 0 & & 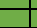 & \\
\hline HPYFYAPELLFFAK & 4.77 & 310 & 1742.89 & 34.8 & 3 & 581.64 & 0 & & & \\
\hline TCVADESAENCDK & 4.77 & 214 & 1498.58 & 11.7 & $\frac{5}{2}$ & 749.79 & 0 & & & \\
\hline LVRPEVDVVMCTAFHDNEETFLK & 4.70 & 40 & 2666.26 & 24.1 & $\frac{2}{4}$ & 667.32 & 0 & & & \\
\hline CCAAADPHECYAK & 4.48 & 99 & 1552.60 & 11.7 & $\frac{7}{2}$ & 776.80 & 0 & & & \\
\hline $\begin{array}{l}\text { CLAAADPHECYAK } \\
\text { VPQVSTPTLVEVSR }\end{array}$ & 4.48 & $\frac{99}{173}$ & $\frac{1532.60}{1511.84}$ & $\frac{11.7}{23.0}$ & $\frac{2}{3}$ & $\frac{717.80}{504.62}$ & $\frac{0}{0}$ & & & \\
\hline HPDYSVVLLLR & 4.27 & 288 & 1311.74 & 27.8 & 3 & 437.92 & 0 & & & \\
\hline YICENODSISSK & 4.17 & 153 & 1443.64 & $\frac{21.0}{13.4}$ & $\frac{5}{2}$ & 722.33 & 0 & & & \\
\hline AVMDDFAAFVEK & 4.11 & $\frac{150}{42}$ & $\frac{1445.04}{1358.63}$ & $\frac{25.4}{25.8}$ & $\frac{2}{2}$ & $\frac{12.03}{679.82}$ & 0 & & & \\
\hline $\begin{array}{l}\text { AVMDLFAAVVK } \\
\text { AVMDDFAAFVK }\end{array}$ & $\frac{4.11}{3.99}$ & $\frac{42}{72}$ & $\frac{1338.63}{1342.63}$ & $\frac{25.8}{29.8}$ & $\frac{2}{2}$ & $\frac{07.82}{671.82}$ & 0 & & - & \\
\hline ETYGEMADCCAK & 3.91 & 50 & 1434.53 & 14.1 & 2 & 717.77 & 0 & & & \\
\hline AAFTECCQAADK & 3.76 & 106 & 1371.57 & 13.2 & $\frac{4}{2}$ & 686.29 & 0 & & & \\
\hline LVNEVTEFAK & 3.71 & 177 & 1149.62 & 21.0 & $\frac{2}{2}$ & 575.31 & 0 & & & \\
\hline LVAASQAALGL & 3.11 & $\frac{171}{210}$ & $\frac{1145.62}{1013.60}$ & 26.6 & $\frac{2}{2}$ & 507.30 & 0 & & & \\
\hline CCTESLVNR & 3.47 & 134 & 1138.50 & $\frac{20.0}{13.5}$ & $\frac{2}{2}$ & 569.75 & 0 & & & \\
\hline ETYGEMADCCAK & 3.45 & 40 & 1450.53 & 11.9 & $\frac{2}{2}$ & 725.77 & 0 & & & \\
\hline SLHTLFGDK & 3.20 & 298 & 1017.54 & 19.0 & $\frac{2}{1}$ & 1017.54 & 0 & & & \\
\hline FQNALLVR & 3.08 & 231 & 960.56 & 21.4 & $\frac{1}{2}$ & 480.78 & 0 & & & \\
\hline ECCEKPLLEK & 3.00 & 112 & 1305.62 & $\frac{1.4}{13.2}$ & $\frac{4}{2}$ & 653.31 & 0 & & & \\
\hline DLGEENFK & 2.99 & 198 & 951.44 & 16.9 & 2 & 476.23 & 0 & & & \\
\hline NECFLQHK & 2.87 & 115 & 1075.50 & 13.1 & $\frac{2}{2}$ & 538.25 & 0 & & - & \\
\hline AACLLPK & 2.78 & 198 & 772.44 & 16.5 & $\frac{2}{2}$ & 386.72 & 0 & & & \\
\hline ETCFAEEGK & 2.76 & 74 & 1070.45 & 12.6 & $\frac{2}{2}$ & 535.73 & 0 & & & \\
\hline YLYEIAR & 2.67 & 226 & $\begin{array}{l}9070.45 \\
927.49\end{array}$ & $\frac{12.0}{20.0}$ & $\frac{2}{2}$ & 464.15 & 0 & & & \\
\hline DDNPNLPR & 2.61 & 89 & 940.45 & 13.7 & $\frac{2}{2}$ & 470.73 & 0 & & & \\
\hline AEFAEVSK & 2.51 & $\frac{69}{129}$ & 880.44 & 13.9 & $\frac{2}{1}$ & 880.44 & 0 & & & \\
\hline $\begin{array}{l}\text { LCTVATLR } \\
\end{array}$ & 2.56 & 244 & 933.52 & 17.1 & 1 & $\begin{array}{l}000.44 \\
933.52 \\
\end{array}$ & 0 & & - & \\
\hline LVTDLTK & 2.56 & 158 & 789.47 & 15.7 & 1 & 789.47 & 0 & & & \\
\hline NYAEAK & 2.43 & 257 & 695.34 & 5.5 & 1 & 695.34 & 0 & & & \\
\hline TYETTLEK & 2.43 & 116 & 984.49 & 13.5 & $\frac{1}{1}$ & 984.49 & 0 & & & \\
\hline CASLQK & 2.40 & 239 & 706.36 & $\frac{13.5}{6.5}$ & $\frac{1}{2}$ & $\frac{90.49}{353.68}$ & 0 & & & \\
\hline $\begin{array}{l}\text { CALQR } \\
\text { QTALVELVK }\end{array}$ & 2.14 & $\frac{239}{223}$ & $\frac{100.30}{1000.60}$ & $\frac{0.5}{22.8}$ & $\frac{2}{1}$ & $\frac{353.08}{1000.60}$ & 0 & & & \\
\hline AWAVAR & 2.09 & 98 & $\frac{200.00}{673.38}$ & 14.9 & $\frac{1}{2}$ & 337.19 & 0 & & & \\
\hline LDELR & 2.03 & 82 & 645.36 & 12.6 & $\frac{2}{2}$ & 323.18 & 0 & & & \\
\hline ADLAK & 2.02 & $\frac{02}{166}$ & 517.30 & $\frac{12.0}{4.9}$ & $\frac{2}{1}$ & 517.30 & 0 & & & \\
\hline $\begin{array}{l}\text { ADLAK } \\
\text { TPVSDR }\end{array}$ & $\frac{2.02}{1.98}$ & $\frac{100}{277}$ & $6 \frac{317.30}{674.35}$ & $\frac{4.9}{6.2}$ & $\frac{1}{1}$ & $\frac{51.30}{674.35}$ & 0 & & & \\
\hline NLGK & 1.68 & 64 & 431.26 & 3.3 & 1 & 431.26 & 0 & & & \\
\hline FGER & 1.58 & 278 & 508.25 & 7.9 & 1 & 508.25 & 0 & & & \\
\hline EQLK & 1.40 & 129 & 517.30 & 4.0 & $\frac{1}{1}$ & 517.30 & 0 & & & \\
\hline QEPER & $\frac{1.46}{1.16}$ & 82 & 658.32 & 3.4 & $\frac{1}{1}$ & 658.32 & 0 & & & \\
\hline DAHK & 0.55 & 37 & 470.24 & 11.1 & 1 & 470.24 & 0 & & & \\
\hline$\overline{~ D L G E E N F K A L V L I A F A Q Y L Q Q C P F E D H V K ~}$ & 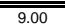 & 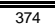 & 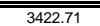 & 41.5 & $=$ & 1141.57 & $\overline{11}$ & & & \\
\hline RMPCAEDYLSVVLNQLCVLHEK & 7.67 & 66 & 2674.31 & $\frac{31.5}{37.0}$ & 3 & 892.11 & 1 & & & \\
\hline LVRPEVDVMCTAFHDNEETFLKK & 6.92 & 99 & 2778.36 & 25.8 & 3 & 926.79 & 1 & & & \\
\hline RMPCAEDYLSVVLNOLCVLHEK & 6.45 & 53 & 2690.31 & 35.8 & $\frac{5}{3}$ & 897.44 & $\frac{1}{1}$ & & & \\
\hline NYAEAKDVFLGMFLYEYAR & 6.16 & 72 & 2300.11 & 36.2 & $\frac{5}{3}$ & 767.38 & $\frac{t}{1}$ & & & \\
\hline MPCAEDYLSVVLNQLCVLHEKTPVSDR & 6.10 & $\frac{12}{66}$ & 3173.54 & 37.4 & $\frac{5}{4}$ & 794.14 & $\frac{1}{1}$ & & & \\
\hline VHTECCHGDLLECADDRADLAK & $\frac{0.10}{6.00}$ & $\frac{60}{82}$ & $\frac{3135.54}{2585.12}$ & $\frac{3.4}{17.3}$ & $\frac{4}{3}$ & $\frac{794.14}{862.38}$ & $\frac{1}{1}$ & & & \\
\hline $\begin{array}{l}\text { VHIECLAGDLLEAAURAALAK } \\
\text { RHPDYSVVLLLR }\end{array}$ & $\frac{6.00}{5.71}$ & $\frac{82}{4554}$ & $\frac{2585.12}{1467.84}$ & $\frac{1.3}{65.6}$ & $\frac{3}{3}$ & $\frac{862.38}{489.95}$ & $\frac{1}{1}$ & & & \\
\hline KVPQVSTPTLVEVSR & 5.32 & 224 & 1639.94 & 20.0 & 3 & 547.32 & 1 & & & \\
\hline RHPYFYAPELLFFAK & 5.27 & 165 & 1899.00 & 32.0 & 3 & 633.67 & 1 & & & \\
\hline LKECCEKPLLEK & 5.07 & 120 & 1546.80 & 13.1 & $\frac{2}{2}$ & 773.90 & 1 & & & \\
\hline QNCELFEQLGEYKFFNALLVR & 4.43 & 11 & $\frac{1540.00}{2599.30}$ & $\frac{1.1}{32.2}$ & $\frac{2}{3}$ & 867.10 & $\frac{1}{1}$ & & & \\
\hline KOTALVELVK & $\frac{4.45}{4.42}$ & $\frac{11}{173}$ & $\frac{2595.00}{1128.70}$ & $\frac{3.2}{19.1}$ & $\frac{5}{3}$ & $\frac{000.10}{376.90}$ & $\frac{1}{1}$ & & & \\
\hline FKDLGEENFK & 4.37 & 132 & 1226.61 & 17.0 & 3 & 409.54 & $\frac{1}{1}$ & & & \\
\hline TCVADESAENCDKSLHTLFGDK & 4.19 & $\frac{152}{12}$ & $\frac{126.01}{2497.10}$ & 20.5 & $\frac{3}{4}$ & $\frac{7025.03}{62.03}$ & $\frac{1}{1}$ & & & \\
\hline ADDKETCFAEEGK & $\frac{4.19}{4.15}$ & $\frac{12}{108}$ & $\frac{2497.10}{1499.63}$ & $\frac{0.5}{12.4}$ & $\frac{4}{3}$ & $\frac{02.03}{500.55}$ & $\frac{1}{1}$ & & & \\
\hline KLVAASQAALGL & 3.52 & 38 & 1141.69 & 23.4 & $\frac{5}{2}$ & 571.35 & 1 & & & \\
\hline QEPERNECFLQHK & 3.52 & 100 & 1714.80 & 13.0 & 2 & 857.90 & 1 & & & \\
\hline KYLYEIAR & 3.39 & 91 & 1055.59 & 17.7 & 2 & 528.30 & 1 & & & \\
\hline LDELRDEGK & 2.97 & 165 & 1074.54 & 12.0 & $\frac{2}{2}$ & 537.78 & 1 & & & \\
\hline EFNAETFTFHADICTLSEKER & 2.24 & $\frac{105}{6}$ & $\frac{1004.54}{2545.17}$ & $\frac{21.0}{26.4}$ & $\frac{2}{4}$ & $\frac{33.18}{637.05}$ & $\frac{1}{1}$ & & & \\
\hline ETCFAEEGKK & 2.15 & 78 & 1198.54 & 11.6 & 2 & 599.77 & 1 & & & \\
\hline NECFLOHKDDNPNLPR & $\frac{2.15}{1.14}$ & $\frac{10}{2}$ & 1996.93 & $\frac{1.0}{16.8}$ & $\frac{2}{3}$ & $\frac{39.11}{666.31}$ & $\frac{1}{1}$ & & & \\
\hline YKAAFTECCQAADK & $\frac{1.14}{0.83}$ & $\frac{2}{3}$ & $\frac{1996.93}{1662.73}$ & $\frac{20.8}{29.7}$ & $\frac{3}{2}$ & $\frac{006.31}{831.86}$ & $\frac{1}{1}$ & & & \\
\hline $\begin{array}{l}\text { PKAAFIELCQAAUK } \\
\text { AWAVARLSQR }\end{array}$ & 0.73 & 21 & 1157.65 & 24.7 & $\frac{4}{2}$ & 579.33 & 1 & & & \\
\hline HPYFYAPELLFFAKR & 0.71 & 12 & 1899.00 & 32.0 & $\frac{2}{2}$ & 950.00 & 1 & & & \\
\hline AAFTECCQAADKAACLLPK & 0.16 & 4 & 2124.99 & 32.6 & 3 & 709.00 & 1 & & & \\
\hline 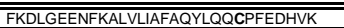 & 8.23 & 71 & 3697.87 & 39.4 & 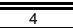 & 925.22 & 2 & & & \\
\hline DVCKNYAEAKDVFLGMFLYEYAR & 6.92 & 40 & 2802.33 & 35.5 & 3 & 934.78 & $\frac{2}{2}$ & & & \\
\hline ADDKETCFAEEGKK & 5.85 & 110 & 1627.73 & 11.6 & 3 & 543.25 & 2 & & & \\
\hline NYAEAKDVFLGMFLYEYARR & 4.78 & 62 & 2456.21 & 33.9 & 3 & 819.41 & $\frac{2}{2}$ & & & \\
\hline AEFAEVSKLVTDLTKVHTECCHGDLLECADDR & 4.38 & 30 & 3718.71 & 30.4 & 5 & 744.55 & $\frac{2}{2}$ & & & \\
\hline HPDYSVVLLLRLAKTYETTLEK & 3.91 & 30 & 2589.43 & 35.2 & 4 & 648.11 & 2 & & & \\
\hline
\end{tabular}


Appendix G2. Peptide identifications of recombinant HSA in SRM 2925 from tandem MS analysis of chymotrypsin digest products. The three randomly selected vials of SRM 2925 (process replicates, Vial 13) were digested and analyzed in triplicate (technical replicates, 1-3). (green: high confidence peptide identifications, yellow: medium confidence peptide identifications, red: low confidence peptide identifications, and black: unidentified peptides)

\begin{tabular}{|c|c|c|c|c|c|c|c|c|c|c|c|c|c|c|c|c|}
\hline \multirow[b]{2}{*}{ Peptide Sequence } & \multirow[b]{2}{*}{$\begin{array}{l}\text { Sequest } \\
\text { XCorr }\end{array}$} & \multirow[b]{2}{*}{ \# PSMs } & \multirow[b]{2}{*}{$\begin{array}{c}\mathrm{m} / \mathrm{z} \\
{[\mathrm{M}+\mathrm{H}]^{+1}} \\
{[\mathrm{Da}]}\end{array}$} & \multirow[b]{2}{*}{$\begin{array}{l}\text { Retention } \\
\text { Time [min] }\end{array}$} & \multirow[b]{2}{*}{ Charge } & \multirow[b]{2}{*}{$\begin{array}{c}\text { Identified } \\
\mathrm{m} / \mathrm{z} \text { [Da] }\end{array}$} & \multirow[b]{2}{*}{$\begin{array}{c}\text { \# Missed } \\
\text { Cleavages }\end{array}$} & \multicolumn{3}{|c|}{ Vial 1} & \multicolumn{3}{|c|}{ Vial 2} & \multicolumn{3}{|c|}{ Vial 3} \\
\hline & & & & & & & & 1 & 2 & 3 & 1 & 2 & 3 & 1 & 2 & 3 \\
\hline AKTCVADESAENCDKSL & 5.74 & 73 & 1897.83 & 13.1 & 3 & 633.28 & 0 & & & & & & & & & \\
\hline GEMADCCAKQEPERNECF & 5.52 & 90 & 2230.86 & 14.7 & 2 & 1115.93 & 0 & & & & & & & & & \\
\hline VEKCCKADDKETCF & 4.86 & 153 & 1789.76 & 11.8 & 2 & 895.38 & 0 & & & & & & & & & \\
\hline GEMADCCAKQEPERNECF & 4.82 & 30 & 2246.86 & 13.4 & 3 & 749.62 & 0 & & & & & & & & & \\
\hline ICENQDSISSKL & 4.42 & 235 & 1393.66 & 18.2 & 2 & 697.34 & 0 & & & & & & & & & \\
\hline EKCCAAADPHECY & 4.21 & 78 & 1610.60 & 12.1 & 3 & 537.54 & 0 & & & & & & & & & \\
\hline TECCQAADKAACL & 3.91 & 126 & 1497.61 & 14.8 & 2 & 749.31 & 0 & & & & & & & & & \\
\hline VESKDVCKNY & 3.6 & 89 & 1241.58 & 11.4 & 2 & 621.30 & 0 & & & & & & & & & \\
\hline TKKVPQVSTPTL & 3.52 & 62 & 1298.77 & 17.4 & 2 & 649.89 & 0 & & & & & & & & & \\
\hline VKHKPKATKEQL & 3.12 & 949 & 1406.85 & 3.9 & 3 & 469.62 & 0 & & & & & & & & & \\
\hline KECCEKPL & 3.04 & 136 & 1063.49 & 11.1 & 3 & 355.17 & 0 & & & & & & & & & \\
\hline KAVMDDF & 2.76 & 91 & 825.38 & 18.9 & 2 & 413.19 & 0 & & & & & & & & & \\
\hline HEKTPVSDRVTKCCTESL & 2.55 & 3 & 2147.02 & 13.5 & 4 & 537.51 & 0 & & & & & & & & & \\
\hline AEAKDVF & 2.47 & 168 & 779.39 & 15.7 & 2 & 390.20 & 0 & & & & & & & & & \\
\hline AEEGKKL & 2.42 & 244 & 774.44 & 4.2 & 2 & 387.72 & 0 & & & & & & & & & \\
\hline IKQNCEL & 2.39 & 1 & 904.46 & 12.8 & 2 & 452.73 & 0 & & & & & & & & & \\
\hline ECADDRADL & 2.38 & 92 & 1064.43 & 13.1 & 2 & 532.72 & 0 & & & & & & & & & \\
\hline EIARRHPY & 2.35 & 138 & 1041.56 & 10.7 & 1 & 1041.56 & 0 & & & & & & & & & \\
\hline VNEVTEF & 2.35 & 175 & 837.40 & 20.5 & 2 & 419.20 & 0 & & & & & & & & & \\
\hline VEVSRNL & 2.34 & 169 & 816.46 & 14.5 & 2 & 408.73 & 0 & & & & & & & & & \\
\hline VEEPQNL & 2.26 & 105 & 828.41 & 16.2 & 2 & 414.71 & 0 & & & & & & & & & \\
\hline AEVSKL & 2.08 & 98 & 646.38 & 12.7 & 1 & 646.38 & 0 & & & & & & & & & \\
\hline VAASQAAL & 2.05 & 130 & 730.41 & 15.5 & 2 & 365.71 & 0 & & & & & & & & & \\
\hline EDHVKL & 1.99 & 93 & 740.39 & 12.3 & 1 & 740.40 & 0 & & & & & & & & & \\
\hline CTVATL & 1.91 & 79 & 664.33 & 18.3 & 1 & 664.34 & 0 & & & & & & & & & \\
\hline HADICTL & 1.9 & 107 & 829.39 & 16.6 & 1 & 829.38 & 0 & & & & & & & & & \\
\hline HDNEETF & 1.85 & 72 & 891.35 & 12.8 & 1 & 891.35 & 0 & & & & & & & & & \\
\hline KAAF & 1.84 & 31 & 436.26 & 10.7 & 1 & 436.25 & 0 & & & & & & & & & \\
\hline \begin{tabular}{|l} 
NAETF \\
\end{tabular} & 1.79 & 82 & 581.26 & 14.4 & 1 & 581.26 & 0 & & & & & & & & & \\
\hline AKVF & 1.71 & 70 & 464.29 & 13.1 & 1 & 464.29 & 0 & & & & & & & & & \\
\hline VPKEF & 1.69 & 116 & 619.34 & 14.0 & 2 & 310.18 & 0 & & & & & & & & & \\
\hline KCASL & 1.56 & 104 & 578.30 & 10.1 & 1 & 578.30 & 0 & & & & & & & & & \\
\hline AVARL & 1.53 & 64 & 529.35 & 12.9 & 2 & 265.18 & 0 & & & & & & & & & \\
\hline GERAF & 1.53 & 63 & 579.29 & 11.7 & 1 & 579.29 & 0 & & & & & & & & & \\
\hline AKTY & 1.5 & 64 & 482.26 & 3.6 & 2 & 241.63 & 0 & & & & & & & & & \\
\hline VNRRPCF & 1.49 & 44 & 948.48 & 12.3 & 2 & 474.75 & 0 & & & & & & & & & \\
\hline EVDETY & 1.45 & 42 & 755.31 & 13.4 & 1 & 755.31 & 0 & & & & & & & & & \\
\hline ARRHPDY & 1.35 & 60 & 914.46 & 3.8 & 3 & 305.49 & 0 & & & & & & & & & \\
\hline VTDL & 1.28 & 47 & 447.24 & 14.0 & 1 & 447.24 & 0 & & & & & & & & & \\
\hline QQCPF & 1.24 & 5 & 679.29 & 16.7 & 1 & 679.29 & 0 & & & & & & & & & \\
\hline RETY & 1.22 & 177 & 568.27 & 5.8 & 1 & 568.27 & 0 & & & & & & & & & \\
\hline SVVL & 1.12 & 29 & 417.27 & 19.1 & 1 & 417.27 & 0 & & & & & & & & & \\
\hline QNAL & 1.02 & 41 & 445.24 & 11.9 & 1 & 445.24 & 0 & & & & & & & & & \\
\hline AKRY & 1.01 & 27 & 537.31 & 10.3 & 2 & 269.16 & 0 & & & & & & & & & \\
\hline GEENF & 0.93 & 1 & 595.24 & 16.2 & 1 & 595.23 & 0 & & & & & & & & & \\
\hline ETTL & 0.86 & 40 & 463.24 & 13.0 & 1 & 463.24 & 0 & & & & & & & & & \\
\hline GDKL & 0.69 & 59 & 432.25 & 5.8 & 1 & 432.24 & 0 & & & & & & & & & \\
\hline
\end{tabular}


Appendix G2 Continued. Peptide identifications of recombinant HSA in SRM 2925 from tandem MS analysis of chymotrypsin digest products. The three randomly selected vials of SRM 2925 (process replicates, Vial 1-3) were digested and analyzed in triplicate (technical replicates, 1-3). (green: high confidence peptide identifications, yellow: medium confidence peptide identifications, red: low confidence peptide identifications, and black: unidentified peptides)

\begin{tabular}{|c|c|c|c|c|c|c|c|c|c|c|c|c|c|c|c|c|}
\hline \multirow[b]{2}{*}{ Peptide Sequence } & \multirow[b]{2}{*}{$\begin{array}{c}\text { Sequest } \\
\text { XCorr }\end{array}$} & \multirow[b]{2}{*}{$\begin{array}{c}\# \\
\text { PSMs }\end{array}$} & \multirow[b]{2}{*}{$\begin{array}{c}\mathrm{m} / \mathbf{z} \\
{[\mathrm{M}+\mathrm{H}]^{+1}} \\
{[\mathrm{Da}]}\end{array}$} & & & & & & ial 1 & & & /ial & & & ial 3 & \\
\hline & & & & $\begin{array}{c}\text { Retention } \\
\text { Time } \\
\text { [min] }\end{array}$ & Charge & $\begin{array}{l}\text { Identified } \\
\mathrm{m} / \mathrm{z}[\mathrm{Da}]\end{array}$ & $\begin{array}{l}\text { \# Missed } \\
\text { Cleavages }\end{array}$ & 1 & 2 & 3 & 1 & 2 & 3 & 1 & 2 & 3 \\
\hline TKKVPQVSTPTLVEVSRNL & 7.47 & 265 & 2096.21 & 23.4 & 3 & 699.41 & 1 & & & & & & & & & \\
\hline ETTLEKCCAAADPHECY & 4.94 & 132 & 2054.83 & 15.6 & 3 & 685.61 & 1 & & & & & & & & & \\
\hline TKVHTECCHGDLL & 3.75 & 33 & 1569.71 & 13.6 & 3 & 523.91 & 1 & & & & & & & & & \\
\hline EVDETYVPKEF & 3.73 & 44 & 1355.64 & 22.5 & 2 & 678.32 & 1 & & & & & & & & & \\
\hline KPLVEEPQNL & 3.64 & 79 & 1166.64 & 19.4 & 2 & 583.83 & 1 & & & & & & & & & \\
\hline ECADDRADLAKY & 3.43 & 77 & 1426.63 & 14.5 & 2 & 713.82 & 1 & & & & & & & & & \\
\hline GDKLCTVATL & 3.35 & 81 & 1077.56 & 20.5 & 2 & 539.29 & 1 & & & & & & & & & \\
\hline IKQNCELF & 3.18 & 191 & 1051.52 & 19.9 & 2 & 526.27 & 1 & & & & & & & & & \\
\hline ICENQDSISSKLKECCEKPL & 3.12 & 25 & 2438.14 & 17.2 & 4 & 610.29 & 1 & & & & & & & & & \\
\hline KDLGEENF & 3.11 & 113 & 951.44 & 16.1 & 2 & 476.23 & 1 & & & & & & & & & \\
\hline AKVFDEF & 3.05 & 156 & 855.42 & 22.4 & 2 & 428.22 & 1 & & & & & & & & & \\
\hline AEAKDVFL & 2.81 & 48 & 892.48 & 21.4 & 2 & 446.74 & 1 & & & & & & & & & \\
\hline SQRFPKAEF & 2.67 & 263 & 1109.57 & 16.7 & 2 & 555.29 & 1 & & & & & & & & & \\
\hline KAVMDDFAAF & 2.64 & 151 & 1114.52 & 27.0 & 2 & 557.77 & 1 & & & & & & & & & \\
\hline TFHADICTL & 2.64 & 173 & 1077.50 & 22.7 & 1 & 1077.51 & 1 & & & & & & & & & \\
\hline SALEVDETY & 2.59 & 133 & 1026.46 & 20.4 & 2 & 513.73 & 1 & & & & & & & & & \\
\hline QQCPFEDHVKL & 2.57 & 13 & 1400.66 & 20.0 & 3 & 467.56 & 1 & & & & & & & & & \\
\hline VTDLTKVHTECCHGDL & 2.57 & 15 & 1884.86 & 15.7 & 4 & 471.97 & 1 & & & & & & & & & \\
\hline ARRHPDYSVVL & 2.43 & 191 & 1312.71 & 16.6 & 2 & 656.86 & 1 & & & & & & & & & \\
\hline LQQCPF & 2.36 & 122 & 792.37 & 19.5 & 1 & 792.37 & 1 & & & & & & & & & \\
\hline DEFKPL & 2.29 & 129 & 748.39 & 20.1 & 2 & 374.70 & 1 & & & & & & & & & \\
\hline NQLCVL & 2.13 & 117 & 746.39 & 23.4 & 2 & 373.70 & 1 & & & & & & & & & \\
\hline KAVMDDFAAF & 2.12 & 19 & 1130.52 & 23.3 & 2 & 565.76 & 1 & & & & & & & & & \\
\hline VLIAF & 1.85 & 172 & 562.36 & 30.9 & 1 & 562.36 & 1 & & & & & & & & & \\
\hline NAETFTF & 1.84 & 25 & 829.37 & 23.2 & 2 & 415.19 & 1 & & & & & & & & & \\
\hline LSVVL & 1.79 & 113 & 530.35 & 26.7 & 1 & 530.36 & 1 & & & & & & & & & \\
\hline EQLGEY & 1.76 & 115 & 738.33 & 16.3 & 1 & 738.33 & 1 & & & & & & & & & \\
\hline RLAKTY & 1.75 & 108 & 751.45 & 10.0 & 1 & 751.45 & 1 & & & & & & & & & \\
\hline KFQNAL & 1.66 & 62 & 720.40 & 15.1 & 1 & 720.41 & 1 & & & & & & & & & \\
\hline QNALL & 1.65 & 51 & 558.32 & 19.8 & 1 & 558.32 & 1 & & & & & & & & & \\
\hline FGDKL & 1.62 & 88 & 579.31 & 15.1 & 2 & 290.16 & 1 & & & & & & & & & \\
\hline HDNEETFL & 1.6 & 59 & 1004.43 & 19.9 & 1 & 1004.43 & 1 & & & & & & & & & \\
\hline IAFAQY & 1.53 & 47 & 712.37 & 22.8 & 1 & 712.37 & 1 & & & & & & & & & \\
\hline LKKY & 1.44 & 120 & 551.36 & 3.5 & 2 & 276.18 & 1 & & & & & & & & & \\
\hline LVRY & 1.33 & 67 & 550.33 & 12.8 & 2 & 275.67 & 1 & & & & & & & & & \\
\hline KALVL & 1.32 & 47 & 543.39 & 18.1 & 1 & 543.39 & 1 & & & & & & & & & \\
\hline QHKDDNPNLPRL & 1.27 & 78 & 1446.74 & 11.6 & 3 & 482.92 & 1 & & & & & & & & & \\
\hline LGMF & 1.2 & 28 & 467.23 & 25.8 & 1 & 467.23 & 1 & & & & & & & & & \\
\hline SVVLL & 1.19 & 27 & 530.35 & 27.0 & 1 & 530.35 & 1 & & & & & & & & & \\
\hline FAKRY & 1.16 & 70 & 684.38 & 10.2 & 1 & 684.38 & 1 & & & & & & & & & \\
\hline APELL & 1.15 & 50 & 542.32 & 22.8 & 1 & 542.32 & 1 & & & & & & & & & \\
\hline VNEVTEFAKTCVADESAENCDKS & 1.02 & 24 & 2716.21 & 26.1 & 4 & 679.81 & 1 & & & & & & & & & \\
\hline LGMF & 1 & 43 & 483.23 & 18.8 & 1 & 483.23 & 1 & & & & & & & & & \\
\hline HTLF & 0.97 & 35 & 517.28 & 17.9 & 1 & 517.28 & 1 & & & & & & & & & \\
\hline SEKERQIKKQTALVEL & 0.88 & 25 & 1900.09 & 15.9 & 4 & 475.78 & 1 & & & & & & & & & \\
\hline GMFL & 0.72 & 4 & 467.23 & 25.9 & 1 & 467.23 & 1 & & & & & & & & & \\
\hline KCASLQKF & 0.32 & 9 & 981.52 & 44.0 & 1 & 981.51 & 1 & & & & & & & & & \\
\hline VRPEVDVMCTAFHDNEETF & 0.31 & 12 & 2312.00 & 40.7 & 2 & 1156.49 & 1 & & & & & & & & & \\
\hline KKYL & 0.25 & 8 & 551.36 & 3.2 & 1 & 551.36 & 1 & & & & & & & & & \\
\hline TKVHTECCHGDLLECADDRADL & 5.88 & 43 & 2615.13 & 18.1 & 3 & 872.38 & 2 & & & & & & & & & \\
\hline AKTCVADESAENCDKSLHTLF & 5.17 & 77 & 2396.09 & 20.6 & 4 & 599.77 & 2 & & & & & & & & & \\
\hline NAETFTFHADICTL & 4.67 & 42 & 1639.74 & 28.3 & 2 & 820.38 & 2 & & & & & & & & & \\
\hline SALEVDETYVPKEF & 4.42 & 172 & 1626.79 & 26.4 & 2 & 813.90 & 2 & & & & & & & & & \\
\hline VTDLTKVHTECCHGDLL & 4.4 & 133 & 1997.94 & 19.4 & 2 & 999.47 & 2 & & & & & & & & & \\
\hline DEFKPLVEEPQNL & 4.37 & 136 & 1557.78 & 26.0 & 2 & 779.39 & 2 & & & & & & & & & \\
\hline AKVFDEFKPL & 4.31 & 176 & 1193.66 & 24.0 & 3 & 398.56 & 2 & & & & & & & & & \\
\hline FGDKLCTVATL & 3.29 & 30 & 1224.63 & 24.5 & 2 & 612.82 & 2 & & & & & & & & & \\
\hline LRLAKTY & 3.01 & 61 & 864.53 & 13.9 & 3 & 288.85 & 2 & & & & & & & & & \\
\hline ARRHPDYSVVLL & 2.46 & 184 & 1425.80 & 21.3 & 2 & 713.40 & 2 & & & & & & & & & \\
\hline LPKLDEL & 2.27 & 30 & 827.49 & 24.0 & 2 & 414.25 & 2 & & & & & & & & & \\
\hline KFQNALL & 2.18 & 113 & 833.49 & 21.1 & 2 & 417.25 & 2 & & & & & & & & & \\
\hline LYEY & 1.6 & 74 & 587.27 & 17.3 & 1 & 587.27 & 2 & & & & & & & & & \\
\hline LLRL & 1.47 & 38 & 514.37 & 20.2 & 2 & 257.69 & 2 & & & & & & & & & \\
\hline GMFLY & 1.3 & 3 & 630.30 & 29.0 & 1 & 630.30 & 2 & & & & & & & & & \\
\hline APELLF & 1.21 & 114 & 689.39 & 31.4 & 2 & 345.20 & 2 & & & & & & & & & \\
\hline SVVLLL & 1.1 & 44 & 643.44 & 32.7 & 1 & 643.44 & 2 & & & & & & & & & \\
\hline YAPELL & 0.93 & 61 & 705.38 & 25.8 & 1 & 705.38 & 2 & & & & & & & & & \\
\hline AEAKDVFLGMF & 0.7 & 33 & 1227.61 & 29.8 & 2 & 614.31 & 2 & & & & & & & & & \\
\hline FFAKRY & 0.51 & 57 & 831.45 & 4.7 & 2 & 416.23 & 2 & & & & & & & & & \\
\hline QKFGERAFKAW & 0.28 & 8 & 1367.72 & 13.2 & 3 & 456.58 & 2 & & & & & & & & & \\
\hline GMFLY & 0.07 & 1 & 646.29 & 75.4 & 1 & 646.28 & 2 & & & & & & & & & \\
\hline
\end{tabular}

